Supporting Information

\title{
Enantioselective Approach to (-)-Hamigeran B via Catalytic Asymmetric Hydrogenation of Racemic Ketone To Assemble the Chiral Core Framework
}

\author{
Han Lin, ${ }^{\dagger}$ Li-Jun Xiao ${ }^{\dagger}$ Min-Jie Zhou, ${ }^{\dagger}$ Hong-Ming Yu,${ }^{\dagger}$ Jian-Hua Xie, ${ }^{*},{ }^{\dagger}$ and Qi-Lin Zhou*, ${ }^{*}, \sharp$
}

\section{Contents:}

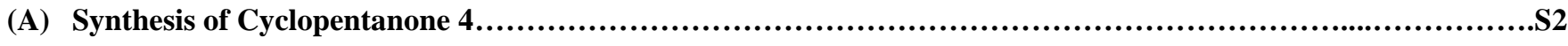

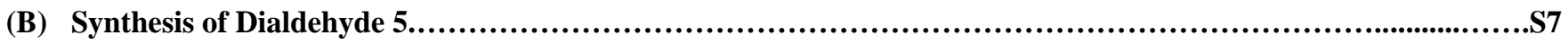

(C) Synthesis of (-)-Hamigeran B and (-)-4-Bromohamigeran B ....................................................S12

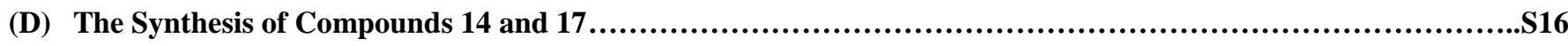

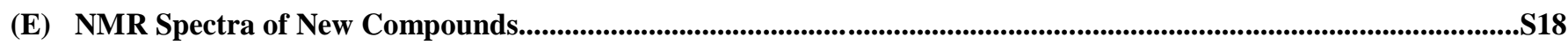

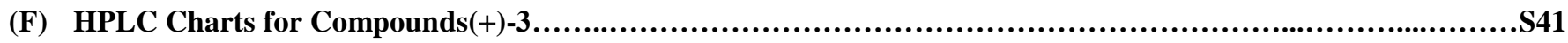

General: All reactions and manipulations which are sensitive to moisture or air were performed in an argon-filled glovebox (VAC DRI-LAB HE 493) or using standard Schlenk techniques. Hydrogen gas (99.999\%) was purchased from Boc Gas Inc., Tianjin. Chemical reagents such as BuLi, $\mathrm{PDC}, \mathrm{SmI}_{2}$, and $\mathrm{Pd}_{2}(\mathrm{dba})_{3} \cdot \mathrm{CHCl}_{3}$ were purchased from Aldrich, Alfa Aesar, and Strem chemical companies. Petroleum ether (PE) refers to the fraction boiling in the $60-90{ }^{\circ} \mathrm{C}$ range. The starting materials such as $2-$ iodocyclopent-2-en-1-one (7), ${ }^{1}$ (3-methoxy-2,5-dimethylphenyl)boronic acid (8) ${ }^{2}$ were prepared according to literature methods. All the solvents concerned were used after purification and dehydration. Anhydrous THF, Et ${ }_{2} \mathrm{O}$, toluene and benzene was distilled from sodium benzophenone ketyl. Anhydrous $\mathrm{CH}_{2} \mathrm{Cl}_{2}, 1,2$-dichloroethane, DMSO, DMF and $i$-PrOH were freshly distilled from calcium hydride. $\mathrm{Et}_{3} \mathrm{~N}$ was distilled from potassium hydroxide. $\mathrm{MeOH}$ and $\mathrm{EtOH}$ were distilled from magnesium. Acetone was distilled from anhydrous potassium carbonate and anhydrous calcium chloride. ${ }^{1} \mathrm{H}$ and ${ }^{13} \mathrm{C}$ NMR spectra were recorded with a Bruker spectrometer at $400 \mathrm{MHz}\left({ }^{1} \mathrm{H} \mathrm{NMR}\right)$ and $100 \mathrm{MHz}\left({ }^{13} \mathrm{C} \mathrm{NMR}\right)$ in $\mathrm{CDCl}_{3}$. Chemical shifts were reported in ppm down field from internal $\mathrm{Me}_{4} \mathrm{Si}$ and external $85 \% \mathrm{H}_{3} \mathrm{PO}_{4}$, respectively. Melting points were measured on a RY-I apparatus and uncorrected. Optical rotations were determined using a Perkin Elmer 341 polarimeter. HRMS were recorded on APEXII and ZAB-HS spectrometer. HPLC analyses were performed using Hewlett Packard Model HP1100 instruments. X-Ray diffraction analysis was performed on Rigaku XtaLAB P200. 
(A) Synthesis of Cyclopentanone 4.

\section{Synthesis of compound $7 .^{1}$}

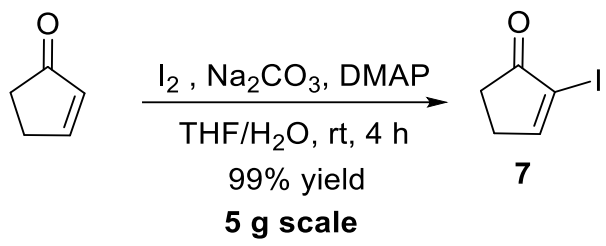

To a solution of cyclopent-2-en-1-one $(5.0 \mathrm{~g}, 61.0 \mathrm{mmol})$ in 1:1 THF- $\mathrm{H}_{2} \mathrm{O}(250 \mathrm{~mL})$ was added $\mathrm{K}_{2} \mathrm{CO}_{3}(10.1 \mathrm{~g}, 73.2 \mathrm{mmol})$, $\mathrm{I}_{2}(23.2 \mathrm{~g}, 91.5 \mathrm{mmol})$ and DMAP $(1.4 \mathrm{~g}, 12.2 \mathrm{mmol})$ successively at room temperature. The reaction mixture was stirred at room temperature for $4 \mathrm{~h}$ to complete the reaction. The reaction mixture was then diluted with EtOAc (100 mL), and washed successively with ice-cooled saturated $\mathrm{Na}_{2} \mathrm{~S}_{2} \mathrm{O}_{3}$ solution $(100 \mathrm{~mL})$. The organic layer was separated and the aqueous layer was extracted with ethyl acetate $(3 \times 50 \mathrm{~mL})$. After dried over $\mathrm{Na}_{2} \mathrm{SO}_{4}$ the organic solution was concentrated in vacuo. The residue was purified by flash-column chromatography on silica gel (ethyl acetate/petroleum = 1:2) to afford 7 (12.5 g, $99 \%$ ) as a white solid $\left(R_{\mathrm{f}}=0.4\right.$, ethyl acetate/petroleum $\left.=1: 5\right) .{ }^{1} \mathrm{H}$ NMR $\left(400 \mathrm{MHz}, \mathrm{CDCl}_{3}\right): \delta 8.00(\mathrm{t}, J=2.9 \mathrm{~Hz}, 1 \mathrm{H}), 2.83-2.69(\mathrm{~m}, 2 \mathrm{H}), 2.52-$ $2.43(\mathrm{~m}, 2 \mathrm{H})$.

\section{Synthesis of compound $8 .^{2}$}

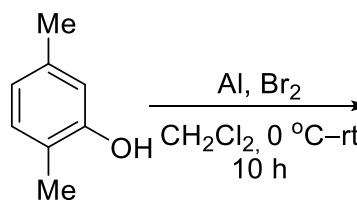

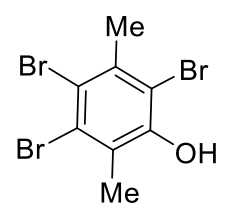

30

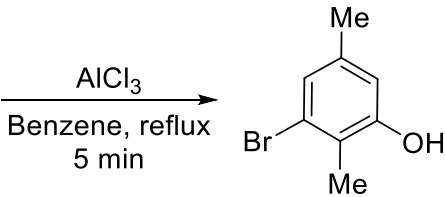

31

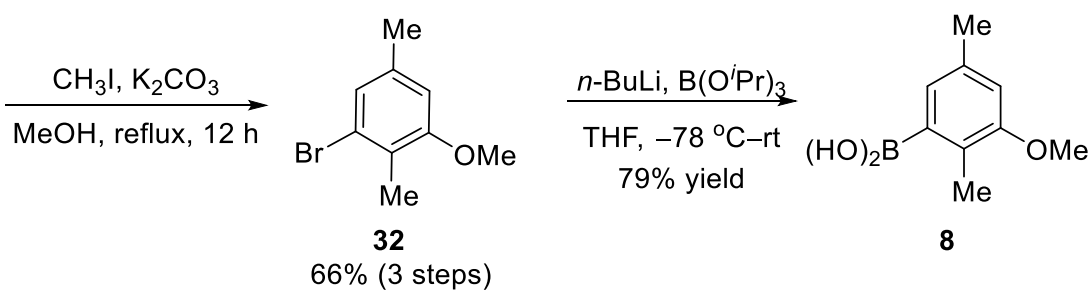

To a flask was charged with $\mathrm{Br}_{2}(100 \mathrm{~mL}, 1.87 \mathrm{~mol})$, and then aluminum $(4.0 \mathrm{~g}, 0.15 \mathrm{mmol})$ was added cautiously in small portions at $0{ }^{\circ} \mathrm{C}$. The mixture was stirred at the same temperature for $2 \mathrm{~h}$ until the sparks ceased. A solution of 2,5-dimethylphenol (40.0 g, $0.33 \mathrm{~mol})$ in $\mathrm{CH}_{2} \mathrm{Cl}_{2}(200 \mathrm{~mL})$ was added dropwise to the mixture over $2 \mathrm{~h}$ (the evolved $\mathrm{HBr}$ gas was trapped by an aqueous $\mathrm{NaOH}$ solution). The resulting mixture was stirred for additional $10 \mathrm{~h}$. The solvent and excess bromine were removed in vacuo, the residue was stirred with $3 \mathrm{M} \mathrm{HCl}(200 \mathrm{~mL})$ for $4 \mathrm{~h}$, and the mixture was filtered to give yellow solid. The yellow solid was dried in vacuo and redissolved in benzene $(180 \mathrm{~mL})$. To the solution was added $\mathrm{AlCl}_{3}(88.1 \mathrm{~g}, 0.66 \mathrm{~mol})$ and the resulting mixture was heated to $80^{\circ} \mathrm{C}$ for $5 \mathrm{~min}$. The mixture was then cooled to room temperature, poured to ice, and diluted with EtOAc $(50 \mathrm{~mL})$. The organic layers were separated, and the aqueous layer was extracted with ethyl acetate $(2 \times 50 \mathrm{~mL})$. The combined organic layers were dried over sodium sulfate. Filtration and concentration of the dried organic layers afforded an oil. The oil was redissolved in $\mathrm{MeOH}(700 \mathrm{~mL})$. After addtion of $\mathrm{K}_{2} \mathrm{CO}_{3}(250 \mathrm{~g}, 1.80 \mathrm{~mol})$ and $\mathrm{CH}_{3} \mathrm{I}(96 \mathrm{~mL}, 1.50 \mathrm{~mol})$, the solution was heated to reflux for $24 \mathrm{~h}$. The solvent was removed in vacuo again, and the yielded residue was redissolved in $\mathrm{H}_{2} \mathrm{O}$ $(200 \mathrm{~mL})$ and $\mathrm{CH}_{2} \mathrm{Cl}_{2}(100 \mathrm{~mL})$. After addition of $2 \mathrm{M} \mathrm{HCl}$ to adjust the $\mathrm{pH}$ to 3, the organic layer was separated, and the aqueous layer was extracted with $\mathrm{CH}_{2} \mathrm{Cl}_{2}(3 \times 50 \mathrm{~mL})$. The combined organic layers were dried over $\mathrm{Na}_{2} \mathrm{SO}_{4}$, filtrated, and concentrated in vacuo. The residue was purified by distillation (bp, $90^{\circ} \mathrm{C} / 0.1$ torr) to afford $32(46 \mathrm{~g}, 66 \%)$ as a yellow liquid ( $R_{\mathrm{f}}=0.89$, ethyl acetate/petroleum = 1:20). ${ }^{1} \mathrm{H}$ NMR $\left(400 \mathrm{MHz} \mathrm{CDCl}_{3}\right) \delta 6.99(\mathrm{~s}, 1 \mathrm{H}), 6.59(\mathrm{~s}, 1 \mathrm{H}), 3.80(\mathrm{~s}, 3 \mathrm{H}), 2.29(\mathrm{~s}, 3 \mathrm{H}), 2.26(\mathrm{~s}, 3 \mathrm{H})$.

The solution of $32(22 \mathrm{~g}, 0.10 \mathrm{~mol})$ in THF $(25 \mathrm{~mL})$ was added to a stirred solution of $n$ - $\mathrm{BuLi}(93 \mathrm{~mL}, 0.20 \mathrm{~mol}, 2.2 \mathrm{M}$ in hexane $)$ in dry THF $(300 \mathrm{~mL})$ at $-78{ }^{\circ} \mathrm{C}$ over $30 \mathrm{~min}$. After the solution was stirred at $-78{ }^{\circ} \mathrm{C}$ for $1 \mathrm{~h}$, a solution of $\mathrm{B}\left(\mathrm{O}{ }^{i} \mathrm{Pr}\right)_{3}(29$ 
g, $0.15 \mathrm{~mol})$ in THF $(25 \mathrm{~mL})$ was added slowly, and the resulting mixture was stirred at $-78{ }^{\circ} \mathrm{C}$ for 30 min. The reaction was quenched by saturated aqueous $\mathrm{NH}_{4} \mathrm{Cl}$ solution $(100 \mathrm{~mL})$ and extracted with EtOAc $(3 \times 100 \mathrm{~mL})$. The organic phase was dried over $\mathrm{Na}_{2} \mathrm{SO}_{4}$, and concentrated in vacuo. The residue was purified by recrystallization (ethyl acetate) to afford $\mathbf{8}(14 \mathrm{~g}$, $79 \%)$ as solid $\left(R_{\mathrm{f}}=0.10\right.$, ethyl acetate/petroleum $\left.=1: 5\right) .{ }^{1} \mathrm{H} \mathrm{NMR}\left(400 \mathrm{MHz}, \mathrm{CDCl}_{3}\right) \delta 7.57(\mathrm{~s}, 1 \mathrm{H}), 6.87(\mathrm{~s}, 1 \mathrm{H}), 3.86(\mathrm{~s}, 3 \mathrm{H}), 2.62(\mathrm{~s}$, $3 \mathrm{H}), 2.40(\mathrm{~s}, 3 \mathrm{H})$.

\section{Synthesis of compound $9 .^{3}$}

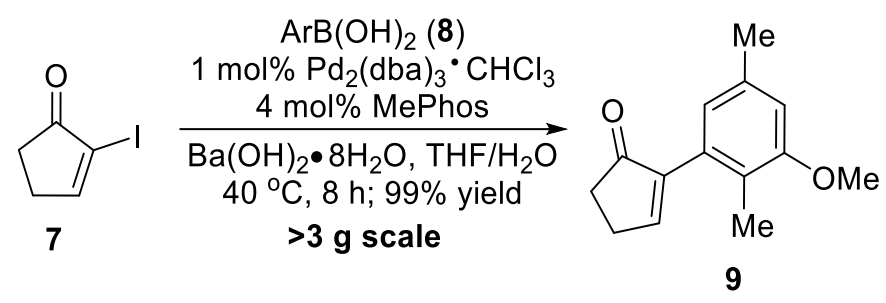

Compound 8 (3.6 g, $20 \mathrm{mmol}), \mathrm{Pd}_{2}(\mathrm{dba})_{3} \cdot \mathrm{CHCl}_{3}(180 \mathrm{mg}, 0.2 \mathrm{mmol})$, MePhos (240 mg, $\left.0.8 \mathrm{mmol}\right)$, and barium hydroxide octahydrate $(19.0 \mathrm{~g}, 60 \mathrm{mmol})$ were added to a solution of 2-iodocyclopent-2-en-1-one 7 (6.4 g, $30 \mathrm{mmol}) \mathrm{in} \mathrm{THF}(320 \mathrm{~mL})$ and distilled water $(66 \mathrm{~mL})$. The resulting mixture was degassed three times and heated to around $40{ }^{\circ} \mathrm{C}$ for $8 \mathrm{~h}$. The reaction mixture was allowed to cool to room temperature, and quenched with saturated aqueous $\mathrm{NH}_{4} \mathrm{Cl}$ solution $(150 \mathrm{~mL})$. The mixture was filtrated to remove the solid, the organic layer was separated, and the aqueous layer was extracted with ethyl acetate $(3 \times 100$ $\mathrm{mL}$ ). The combined organic layers were dried over $\mathrm{Na}_{2} \mathrm{SO}_{4}$, filtrated and concentrated in vacuo. The residue was purified by chromatography on silical gel (ethyl acetate/petroleum $=1: 5)$ to afford $9(4.34 \mathrm{~g}, 99 \%)$ as a yellow liquid $\left(R_{\mathrm{f}}=0.42\right.$, ethyl acetate/petroleum = 1:5). ${ }^{1} \mathrm{H}$ NMR $\left(300 \mathrm{MHz} \mathrm{CDCl}_{3}\right) \delta 7.56(\mathrm{t}, J=2.8 \mathrm{~Hz}, 1 \mathrm{H}), 6.66(\mathrm{~s}, 1 \mathrm{H}), 6.57(\mathrm{~s}, 1 \mathrm{H}), 3.81(\mathrm{~s}, 3 \mathrm{H}), 2.81-$ $2.71(\mathrm{~m}, 2 \mathrm{H}), 2.62-2.52(\mathrm{~m}, 2 \mathrm{H}), 2.32(\mathrm{~s}, 3 \mathrm{H}), 2.02(\mathrm{~s}, 3 \mathrm{H}) .{ }^{13} \mathrm{C} \mathrm{NMR}\left(101 \mathrm{MHz}, \mathrm{CDCl}_{3}\right) \delta 207.7(1 \mathrm{C}, \mathrm{C}=\mathrm{O}), 161.0(1 \mathrm{C},=\mathrm{CH})$, 157.6 (1C, Ar-C), 146.4 (1C, C), 135.8 (1C, Ar-C), 132.8 (1C, Ar-C), 121.9 (1C, Ar-C), 121.9 (1C, Ar-CH), 111.0 (1C, Ar-CH), $55.4\left(1 \mathrm{C}, \mathrm{OCH}_{3}\right), 34.7\left(1 \mathrm{C}, \mathrm{CH}_{2}\right), 26.8\left(1 \mathrm{C}, \mathrm{CH}_{2}\right), 21.3\left(1 \mathrm{C}, \mathrm{CH}_{3}\right), 12.8\left(1 \mathrm{C}, \mathrm{CH}_{3}\right)$. IR $(\mathrm{KBr}): v_{\max } 2994,2923,2858,2838,1703$,

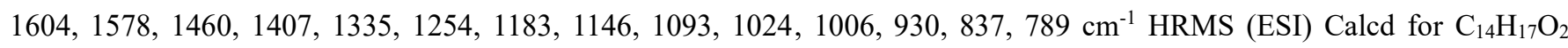
$\left([\mathrm{M}+\mathrm{H}]^{+}\right): 217.1223$; Found: 217.1226 .

\section{Synthesis of compound $10 .^{4}$}

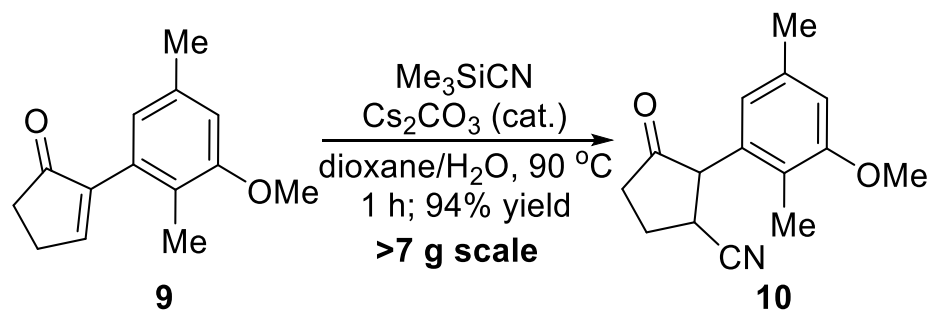

To a solution of compound 9 (7.2 g, $33.1 \mathrm{mmol})$ in dioxane $(55 \mathrm{~mL})$ was added $\mathrm{Cs}_{2} \mathrm{CO}_{3}(55 \mathrm{mg}, 0.17 \mathrm{mmol}), \mathrm{Me} 3 \mathrm{SiCN}(6.3$ $\mathrm{mL}, 49.7 \mathrm{mmol})$, and $\mathrm{H}_{2} \mathrm{O}(2.4 \mathrm{~mL}, 132.4 \mathrm{mmol})$. The reaction mixture was stirred at $90-100{ }^{\circ} \mathrm{C}$ for $1 \mathrm{~h}$ to complete the reaction, $\mathrm{H}_{2} \mathrm{O}(10 \mathrm{~mL})$ was then added at room temperature to quench the reaction. The resulting mixture was extracted with EtOAc $(3 \times$ $20 \mathrm{~mL}$ ) (Caution! HCN generated in the reaction mixture is highly toxic. Those operations should be conducted in a wellventilated hood). The combined organic layers were dried over sodium sulfate, filtrated and concentrated in vacuo. The ${ }^{1} \mathrm{H}$ NMR analysis of the crude product showed that the Michael addition products are the mixture of trans/cis in a ratio of $>10: 1$. The crude product was purified by flash-column chromatography on silica gel (ethyl acetate/petroleum $=1: 3)$ to afford trans-10 $(7.6$ 
$\mathrm{g}, 94 \%)$ as a white solid $\left(R_{\mathrm{f}}=0.21\right.$, ethyl acetate/petroleum $\left.=1: 5\right) . \mathrm{Mp}: 140-142{ }^{\circ} \mathrm{C} ;{ }^{1} \mathrm{H} \mathrm{NMR}(400 \mathrm{MHz}, \mathrm{CDCl} 3) \delta 6.63(\mathrm{~s}, 1 \mathrm{H})$, $6.32(\mathrm{~s}, 1 \mathrm{H}), 3.85(\mathrm{~d}, J=11.6 \mathrm{~Hz}, 1 \mathrm{H}), 3.81(\mathrm{~s}, 3 \mathrm{H}), 3.20(\mathrm{td}, J=11.3,6.3 \mathrm{~Hz}, 1 \mathrm{H}), 2.74-2.59(\mathrm{~m}, 2 \mathrm{H}), 2.50-2.39(\mathrm{~m}, 1 \mathrm{H}), 2.36-$ $2.23(\mathrm{~m}, 4 \mathrm{H}), 2.16(\mathrm{~s}, 3 \mathrm{H}) .{ }^{13} \mathrm{C}$ NMR $\left(101 \mathrm{MHz}, \mathrm{CDCl}_{3}\right) \delta 212.2(1 \mathrm{C}, \mathrm{C}=\mathrm{O}), 157.9(1 \mathrm{C}, \mathrm{Ar}-\mathrm{C}), 136.7$ (1C, Ar-C), $134.3(1 \mathrm{C}, \mathrm{Ar}-$ C), 123.1 (1C, C), 120.3 (1C, Ar-C), 120.2 (1C, Ar-CH), $111.1(1 \mathrm{C}, \mathrm{Ar}-\mathrm{CH}), 56.4(1 \mathrm{C}, \mathrm{CH}), 55.5\left(1 \mathrm{C}, \mathrm{OCH}_{3}\right), 37.2\left(1 \mathrm{C}, \mathrm{CH}_{2}\right)$, 34.0 (1C, $\mathrm{CH}), 25.4\left(1 \mathrm{C}, \mathrm{CH}_{2}\right), 21.4\left(1 \mathrm{C}, \mathrm{CH}_{3}\right), 11.8\left(1 \mathrm{C}, \mathrm{CH}_{3}\right)$. IR (KBr): v $\max 3056,2938,2839,2243,1749,1612,1583,1462$, 1415, 1330, 1281, 1145, 837, $734 \mathrm{~cm}^{-1} \mathrm{HRMS}$ (ESI) Calcd for $\mathrm{C}_{15} \mathrm{H}_{18} \mathrm{NO}_{2}\left([\mathrm{M}+\mathrm{H}]^{+}\right)$: 244.1332; Found: 244.1334.

\section{Synthesis of compound trans-2.}

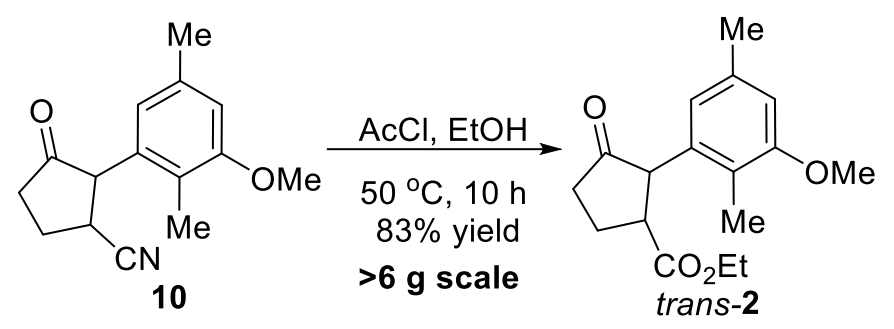

To a sealing tube was added compound trans-10 $(6.1 \mathrm{~g}, 25 \mathrm{mmol}), \mathrm{EtOH}(40 \mathrm{~mL})$, and $\mathrm{AcCl}(10 \mathrm{~mL})$ successively at $0{ }^{\circ} \mathrm{C}$. The sealed tube was heated at $50{ }^{\circ} \mathrm{C}$ for $10 \mathrm{~h}$ to complete the reaction. The reaction mixture was cooled to room temperature, and $\mathrm{H}_{2} \mathrm{O}(10 \mathrm{~mL})$ was added. After addition of $\mathrm{NaHCO}_{3}$ to adjust the $\mathrm{pH}$ to $>7$, the mixture was extracted with EtOAc $(3 \times 20$ $\mathrm{mL}$ ). The combined organic layers were dried over sodium sulfate, filtrated, and concentrated in vacuo. The residue was purified by chromatography on silica gel (ethyl acetate/petroleum $=1: 5)$ to afford trans-2 $(6.0 \mathrm{~g}, 83 \%)$ as a yellow oil, which could be further purified by distillation (bp. $162{ }^{\circ} \mathrm{C} / 0.08$ torr) $\left(R_{\mathrm{f}}=0.42\right.$, ethyl acetate/petroleum $\left.=1: 5\right) .{ }^{1} \mathrm{H} \mathrm{NMR}\left(400 \mathrm{MHz}, \mathrm{CDCl}{ }_{3}\right) \delta$ $6.58(\mathrm{~s}, 1 \mathrm{H}), 6.37(\mathrm{~s}, 1 \mathrm{H}), 4.17-4.06(\mathrm{~m}, 2 \mathrm{H}), 3.95(\mathrm{~d}, J=11.2 \mathrm{~Hz}, 1 \mathrm{H}), 3.79(\mathrm{~s}, 3 \mathrm{H}), 3.27(\mathrm{td}, J=11.0,6.2 \mathrm{~Hz}, 1 \mathrm{H}), 2.70-2.57$ $(\mathrm{m}, 1 \mathrm{H}), 2.53-2.36(\mathrm{~m}, 2 \mathrm{H}), 2.27(\mathrm{~s}, 3 \mathrm{H}), 2.20-2.03(\mathrm{~m}, 4 \mathrm{H}), 1.20(\mathrm{t}, J=7.2 \mathrm{~Hz}, 3 \mathrm{H}) .{ }^{13} \mathrm{C} \mathrm{NMR}\left(101 \mathrm{MHz}, \mathrm{CDCl}_{3}\right) \delta 215.0(1 \mathrm{C}$, $\mathrm{C}=\mathrm{O}), 173.3(1 \mathrm{C}, \mathrm{OC}=\mathrm{O}), 157.3$ (1C, Ar-C), 136.3 (1C, Ar-C), 135.6 (1C, Ar-C), 122.5 (1C, Ar-C), 120.5 (1C, Ar-CH), 109.9 $(1 \mathrm{C}, \mathrm{Ar}-\mathrm{CH}), 60.5\left(1 \mathrm{C}, \mathrm{OCH}_{2}\right), 56.0(1 \mathrm{C}, \mathrm{CH}), 55.0\left(1 \mathrm{C}, \mathrm{OCH}_{3}\right), 48.8(1 \mathrm{C}, \mathrm{CH}), 37.4\left(1 \mathrm{C}, \mathrm{CH}_{2}\right), 24.7\left(1 \mathrm{C}, \mathrm{CH}_{2}\right), 21.0\left(1 \mathrm{C}, \mathrm{CH}_{3}\right)$, $13.7\left(1 \mathrm{C}, \mathrm{CH}_{3}\right), 11.3\left(1 \mathrm{C}, \mathrm{CH}_{3}\right) . \mathrm{IR}(\mathrm{KBr}): v_{\max } 3057,2982,2939,1730,1611,1582,1462,1267,1198,1146,1068,739,704$ $\mathrm{cm}^{-1}$ HRMS (ESI) Calcd for $\mathrm{C}_{17} \mathrm{H}_{23} \mathrm{O}_{4}\left([\mathrm{M}+\mathrm{H}]^{+}\right)$: 291.1591; Found: 291.1594.

\section{Synthesis of compound 3 via asymmetric hydrogenation.}

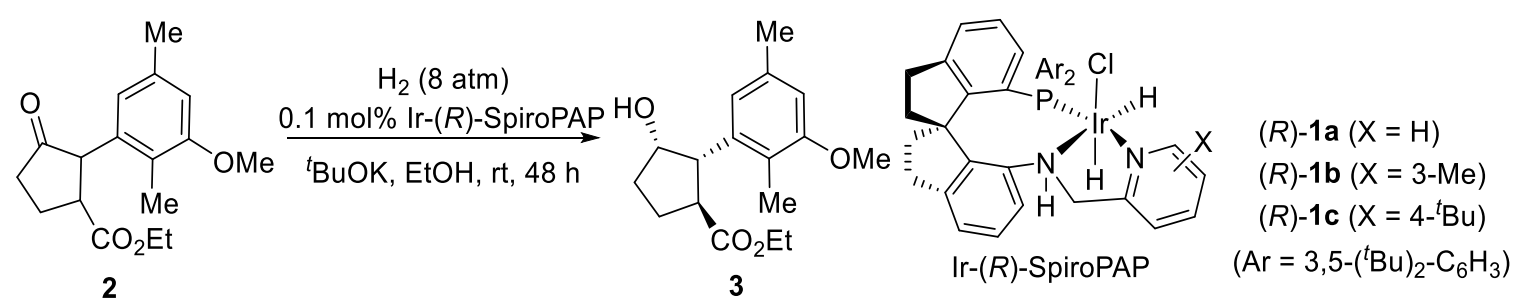

General procedure: To a $10 \mathrm{~mL}$ Schlenk tube was added the catalyst precursor $[\operatorname{Ir}(\mathrm{COD}) \mathrm{Cl}]_{2}(0.67 \mathrm{mg}, 0.001 \mathrm{mmol})$, $(R)$-SpiroPAP ligand $(1.6 \mathrm{mg}, 0.002 \mathrm{mmol})$, and anhydrous EtOH $(0.5 \mathrm{~mL})$ under nitrogen atmosphere. The mixture was stirred at room temperature $\left(25-30^{\circ} \mathrm{C}\right)$ for $1.0 \mathrm{~h}$ to give a clear yellow solution, then half of the solution was transferred to a vessel. The vessel was palced in an autoclave and pressurized to $10 \mathrm{~atm}$ of $\mathrm{H}_{2}$ and stirred at room temperature for $5 \mathrm{~min}$. After releasing the hydrogen pressure, the solution of trans-2 $(290 \mathrm{mg}, 1 \mathrm{mmol})$ in $\mathrm{EtOH}(1 \mathrm{~mL})$ and a solution of $t$ - BuOK $(9 \mathrm{mg}, 0.08 \mathrm{mmol})$ in $\mathrm{EtOH}(0.5 \mathrm{~mL})$ was added successively. The vessel was pressurized to $8 \mathrm{~atm}$ of $\mathrm{H}_{2}$ again, and the reaction mixture was stirred at room temperature for $4 \mathrm{~h}$. After releasing the hydrogen pressure, the reaction mixture was continued to stirr at room temperature for about $44 \mathrm{~h}$ (monitered by TLC until the small amount of cis,cis-isomer was converted to trans,cis-isomer). Saturated aquenous $\mathrm{NH}_{4} \mathrm{Cl}$ solution $(10 \mathrm{~mL})$ was added, and the resulting mixture was extracted with EtOAc $(3 \times 20 \mathrm{~mL})$. The 
combined organic layers were dried over sodium sulfate, filtrated, and concentrated in vacuo. The residue was purified by flashcolumn chromatography on silical gel (ethyl acetate/petroleum $=1: 5)$ to afford $(+)-3$ as a yellow oil $\left(R_{\mathrm{f}}=0.42\right.$, ethyl acetate/petroleum = 1:5). ${ }^{1} \mathrm{H} \mathrm{NMR}\left(400 \mathrm{MHz}^{\mathrm{CDCl}}{ }_{3}\right) \delta 6.74(\mathrm{~s}, 1 \mathrm{H}), 6.60(\mathrm{~s}, 1 \mathrm{H}), 4.27(\mathrm{t}, J=4.4 \mathrm{~Hz}, 1 \mathrm{H}), 4.03(\mathrm{~m}, 2 \mathrm{H}), 3.79$ $(\mathrm{s}, 3 \mathrm{H}), 3.63(\mathrm{dd}, J=11.4,4.4 \mathrm{~Hz}, 1 \mathrm{H}), 3.39(\mathrm{dt}, J=11.2,8.6 \mathrm{~Hz}, 1 \mathrm{H}), 2.40-2.25(\mathrm{~m}, 4 \mathrm{H}), 2.22-2.08(\mathrm{~m}, 4 \mathrm{H}), 1.97-1.81(\mathrm{~m}$, $2 \mathrm{H}), 1.14(\mathrm{t}, J=7.2 \mathrm{~Hz}, 3 \mathrm{H}) .{ }^{13} \mathrm{C} \mathrm{NMR}\left(101 \mathrm{MHz}, \mathrm{CDCl}_{3}\right) \delta 175.6(1 \mathrm{C}, \mathrm{OC}=\mathrm{O}), 157.6(1 \mathrm{C}, \mathrm{Ar}-\mathrm{C}), 135.9(1 \mathrm{C}, \mathrm{Ar}-\mathrm{C}), 135.6(1 \mathrm{C}$, Ar-C), 122.3 (1C, Ar-C), 120.3 (1C, Ar-CH), 110.1 (1C, Ar-CH), 73.7 (1C, CH), $60.2\left(1 \mathrm{C}, \mathrm{OCH}_{2}\right), 55.3\left(1 \mathrm{C}, \mathrm{OCH}_{3}\right), 51.7(1 \mathrm{C}$, $\mathrm{CH}), 45.5(1 \mathrm{C}, \mathrm{CH}), 32.9\left(1 \mathrm{C}, \mathrm{CH}_{2}\right), 27.7\left(1 \mathrm{C}, \mathrm{CH}_{2}\right), 21.6\left(1 \mathrm{C}, \mathrm{CH}_{3}\right), 14.0\left(1 \mathrm{C}, \mathrm{CH}_{3}\right), 10.8\left(1 \mathrm{C}, \mathrm{CH}_{3}\right) . \mathrm{IR}(\mathrm{KBr}): v_{\max } 3438,2959$, 2874, 1728, 1611, 1582, 1462, 1286, 1202, 1144, 1111, 1094, 1076, 1028, $833 \mathrm{~cm}^{-1}$ HRMS (ESI) Calcd for $\mathrm{C}_{17} \mathrm{H}_{25} \mathrm{O}_{4}\left([\mathrm{M}+\mathrm{H}]^{+}\right)$: 293.1747; Found: 293.1752.

The experiment data of optimizing the hydrogenation conditions see Table S1.

Table S1. Optimizing the hydrogenation conditions. ${ }^{a}$

\begin{tabular}{cccccc}
\hline Entry & Ir- $(R)$-SpiroPAP & {$[$ Base $](\mathrm{mmol} / \mathrm{mL})$} & ${\text { Conv. }(\%)^{b}}^{b}$ & ${\text { Yield }(\%)^{c}}^{c}$ & ${\text { Ee }(\%)^{d}}^{d}$ \\
\hline 1 & $(R)-\mathbf{1 a}$ & 0.04 & 100 & 97 & 95 \\
2 & $(R)-\mathbf{1 b}$ & 0.04 & 100 & 97 & 96 \\
3 & $(R)-\mathbf{1 c}$ & 0.04 & 100 & 97 & 97 \\
4 & $(R)-\mathbf{1 c}$ & 0.02 & 100 & 90 & 97 \\
5 & $(R)-\mathbf{1 c}$ & 0.06 & 100 & 97 & 97 \\
\hline
\end{tabular}

Notes: ${ }^{a}$ Reaction conditions: $1 \mathrm{mmol} \mathrm{scale},[\mathrm{rac}-2]=0.5 \mathrm{M}, 25-30{ }^{\circ} \mathrm{C}, 8 \mathrm{~atm} \mathrm{H}, 48 \mathrm{~h} .{ }^{b}$ Determined by ${ }^{1} \mathrm{H}$ NMR. ${ }^{c}$ Isolated yield. ${ }^{d}$ Determined by HPLC on chiral AD-H column.

HPLC conditions: Chiralcel AD-H column $(25 \mathrm{~cm} \times 0.46 \mathrm{~cm}$ ID); $n$-hexane $/ 2$-propanol = 95:5; temp, rt; flow rate = 1.0 $\mathrm{mL} / \mathrm{min} ; 220 \mathrm{~nm} \mathrm{UV}$ detector; $t_{\mathrm{R}}(1 S, 2 S, 3 S)=8.15 \mathrm{~min}$ and $t_{\mathrm{R}}(1 R, 2 R, 3 R)=11.395 \mathrm{~min}$.

Asymmetric hydrogenation of trans-2 in gram-scale.
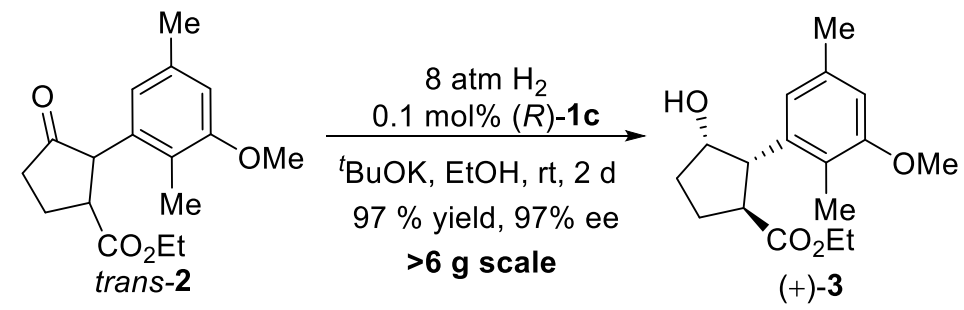

To a $20 \mathrm{~mL}$ Schlenk tube was added the catalyst precursor $[\operatorname{Ir}(\mathrm{COD}) \mathrm{Cl}]_{2}(7.5 \mathrm{mg}, 0.011 \mathrm{mmol}),(R)-\mathrm{SpiroPAP}-4-{ }^{t} \mathrm{Bu}(18$ $\mathrm{mg}, 0.0224 \mathrm{mmol})$, and anhydrous EtOH $(5 \mathrm{~mL})$ under nitrogen atmosphere. The mixture was stirred at room temperature $\left(25-30{ }^{\circ} \mathrm{C}\right.$ ) for $1.0 \mathrm{~h}$ to give a clear yellow solution, then stirred under atmospheric $\mathrm{H}_{2}$ pressure (balloon) for $1 \mathrm{~h}$ until the yellow color faded. Then the solution was transferred to a vessel, and a solution of trans $-2(6.5 \mathrm{~g}, 22.4 \mathrm{mmol}) \mathrm{in} \mathrm{EtOH} \mathrm{(40} \mathrm{mL)} \mathrm{and} \mathrm{a}$ solution of $t$-BuOK (224 mg, $2 \mathrm{mmol})$ in $\mathrm{EtOH}(5 \mathrm{~mL})$ were added. The vessel was pressurized to 10 atm of $\mathrm{H}_{2}$ again, and the reaction mixture was stirred at room temperature for $48 \mathrm{~h}$. After releasing the hydrogen pressure a saturated aquenous $\mathrm{NH}_{4} \mathrm{Cl}$ solution $(10 \mathrm{~mL})$ was added, and the resulting mixture was extracted with EtOAc $(3 \times 20 \mathrm{~mL})$. The combined organic layers were dried over sodium sulfate, filtrated, and concentrated in vacuo. The residue was purified by flash-column chromatography on silical gel (ethyl acetate/petroleum $=1: 5)$ to afford $(+)-3\left(6.4 \mathrm{~g}, 97 \%\right.$ yield, 97\% ee) as a yellow oil. $[\alpha]_{\mathrm{D}}^{26}+129.6(c 1.0, \mathrm{CHCl} 3)$ ${ }^{1} \mathrm{H}$ NMR $\left(400 \mathrm{MHz}, \mathrm{CDCl}_{3}\right) \delta 6.74(\mathrm{~s}, 1 \mathrm{H}), 6.60(\mathrm{~s}, 1 \mathrm{H}), 4.27(\mathrm{t}, J=4.4 \mathrm{~Hz}, 1 \mathrm{H}), 4.03(\mathrm{~m}, 2 \mathrm{H}), 3.79(\mathrm{~s}, 3 \mathrm{H}), 3.63(\mathrm{dd}, J=11.4$, $4.4 \mathrm{~Hz}, 1 \mathrm{H}), 3.39(\mathrm{dt}, J=11.2,8.6 \mathrm{~Hz}, 1 \mathrm{H}), 2.40-2.25(\mathrm{~m}, 4 \mathrm{H}), 2.22-2.08(\mathrm{~m}, 4 \mathrm{H}), 1.97-1.81(\mathrm{~m}, 2 \mathrm{H}), 1.14(\mathrm{t}, J=7.2 \mathrm{~Hz}, 3 \mathrm{H})$. ${ }^{13} \mathrm{C} \mathrm{NMR}\left(101 \mathrm{MHz}, \mathrm{CDCl}_{3}\right) \delta 175.6(1 \mathrm{C}, \mathrm{OC}=\mathrm{O}), 157.6$ (1C, Ar-C), 135.9 (1C, Ar-C), 135.6 (1C, Ar-C), 122.3 (1C, Ar-C), $120.3(1 \mathrm{C}, \mathrm{Ar}-\mathrm{CH}), 110.1(1 \mathrm{C}, \mathrm{Ar}-\mathrm{CH}), 73.7(1 \mathrm{C}, \mathrm{CH}), 60.2\left(1 \mathrm{C}, \mathrm{OCH}_{2}\right), 55.3\left(1 \mathrm{C}, \mathrm{OCH}_{3}\right), 51.7(1 \mathrm{C}, \mathrm{CH}), 45.5(1 \mathrm{C}, \mathrm{CH}), 32.9$ $\left(1 \mathrm{C}, \mathrm{CH}_{2}\right), 27.7\left(1 \mathrm{C}, \mathrm{CH}_{2}\right), 21.6\left(1 \mathrm{C}, \mathrm{CH}_{3}\right), 14.0\left(1 \mathrm{C}, \mathrm{CH}_{3}\right), 10.8\left(1 \mathrm{C}, \mathrm{CH}_{3}\right)$. IR $(\mathrm{KBr}): v_{\max } 3438,2959,2874,1728,1611,1582$, 
1462, 1286, 1202, 1144, 1111, 1094, 1076, 1028, $833 \mathrm{~cm}^{-1}$ HRMS (ESI) Calcd for $\mathrm{C}_{17} \mathrm{H}_{25} \mathrm{O}_{4}\left([\mathrm{M}+\mathrm{H}]^{+}\right): 293.1747$; Found: 293.1752 .

\section{Synthesis of compound 12. ${ }^{5}$}

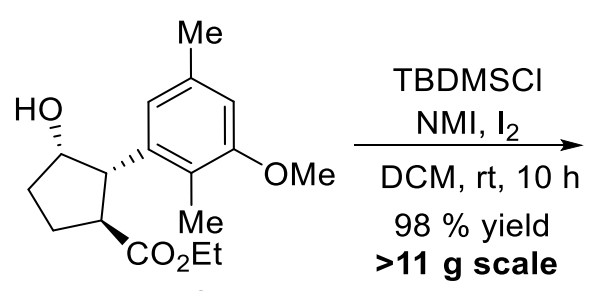

$(+)-3$

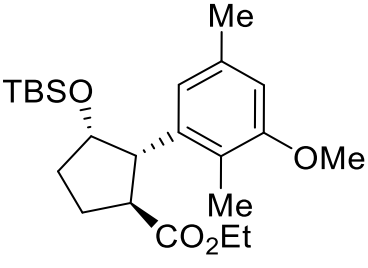

12

To a solution of $(+)-3(11.4 \mathrm{~g}, 38.8 \mathrm{mmol})$ in DCM $(120 \mathrm{~mL})$ was added $N$-methylimidazole $(9.6 \mathrm{~g}, 0.12 \mathrm{~mol})$, and iodine (29.4 g, $0.12 \mathrm{~mol})$, and TBDMSCl $(8.8 \mathrm{~g}, 58.2 \mathrm{mmol})$. The reaction mixture was stirred at room temperature for $10 \mathrm{~h}$. The solvent was removed in vacuo, the residue was redissolved in EtOAc, washed with saturated aqueous $\mathrm{Na}_{2} \mathrm{~S}_{2} \mathrm{O}_{3}$ solution (150 mL), dried over anhydrous $\mathrm{Na}_{2} \mathrm{SO}_{4}$, and concentrated in vacuo. The residue was purified by chromatography on silical gel (ethyl acetate/petroleum $=1: 20)$ to afford $12(15.4 \mathrm{~g}, 98 \%)$ as a colorless oil $\left(R_{\mathrm{f}}=0.41\right.$, ethyl acetate/petroleum $\left.=1: 20\right)$. $[\alpha]_{\mathrm{D}}^{26}+129.6$ (c 1.0, $\left.\mathrm{CHCl}_{3}\right) .{ }^{1} \mathrm{H}$ NMR $\left(400 \mathrm{MHz}, \mathrm{CDCl}_{3}\right) \delta 6.76(\mathrm{~s}, 1 \mathrm{H}), 6.53(\mathrm{~s}, 1 \mathrm{H}), 4.25(\mathrm{~s}, 1 \mathrm{H}), 4.03(\mathrm{q}, J=7.0 \mathrm{~Hz}, 2 \mathrm{H}), 3.78(\mathrm{~s}, 3 \mathrm{H})$, 3.59-3.38 (m, 2H), 2.40-2.21 (m, 4H), 2.20-2.03 (m, 4H), 1.93-1.70 (m, 2H), 1.15 (t, J = 7.0 Hz, 3H), 0.73 (s, 9H), -0.27 (s, $3 \mathrm{H}),-0.51$ (s, 3H). ${ }^{13} \mathrm{C}$ NMR (101 MHz, $\left.\mathrm{CDCl}_{3}\right) \delta 176.2(1 \mathrm{C}, \mathrm{OC}=\mathrm{O}), 157.2(1 \mathrm{C}, \mathrm{Ar}-\mathrm{C}), 137.2(1 \mathrm{C}, \mathrm{Ar}-\mathrm{C}), 134.2(1 \mathrm{C}, \mathrm{Ar}-\mathrm{C})$, 122.2 (1C, Ar-CH), 121.7 (1C, Ar-C), 109.5 (1C, Ar-CH), $74.9(1 \mathrm{C}, \mathrm{CH}), 60.1\left(1 \mathrm{C}, \mathrm{OCH}_{2}\right), 55.6\left(1 \mathrm{C}, \mathrm{OCH}_{3}\right), 51.6(1 \mathrm{C}, \mathrm{CH})$, $45.8(1 \mathrm{C}, \mathrm{CH}), 35.2\left(1 \mathrm{C}, \mathrm{CH}_{2}\right), 27.5\left(1 \mathrm{C}, \mathrm{CH}_{2}\right), 25.7\left(3 \mathrm{C}, \mathrm{CH}_{3}\right), 21.5\left(1 \mathrm{C}, \mathrm{CH}_{3}\right), 17.9(1 \mathrm{C}, \mathrm{C}), 14.1\left(1 \mathrm{C}, \mathrm{CH}_{3}\right), 10.8\left(1 \mathrm{C}, \mathrm{CH}_{3}\right)$, $-5.6\left(1 \mathrm{C}, \mathrm{CH}_{3}\right),-5.8\left(1 \mathrm{C}, \mathrm{CH}_{3}\right) . \mathrm{IR}(\mathrm{KBr}): v_{\max } 2953,2930,2902,2855,1731,1613,1582,1463,1295,1253,1160,1143,1092$, 1038, 832, 774, $745 \mathrm{~cm}^{-1} \mathrm{HRMS}(\mathrm{ESI})$ Calcd for $\mathrm{C}_{23} \mathrm{H}_{39} \mathrm{O}_{4} \mathrm{Si}\left([\mathrm{M}+\mathrm{H}]^{+}\right)$: 407.2612; Found: 407.2616.

\section{Synthesis of compound 13.}

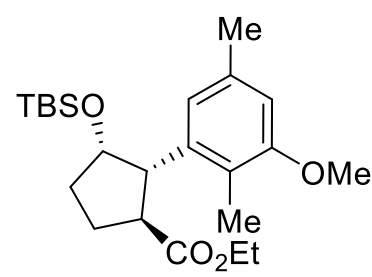

12

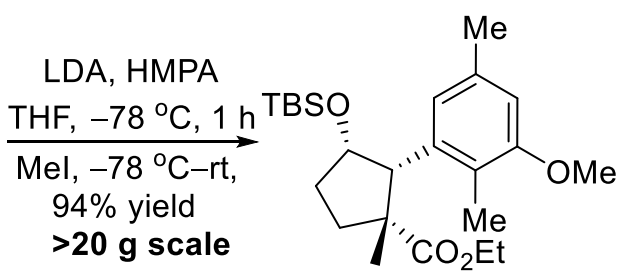

13

To a stirred solution of $N, N$-diisopropylamine $(86 \mathrm{~mL}, 605 \mathrm{mmol})$ in dry THF $(400 \mathrm{~mL})$ at $-78^{\circ} \mathrm{C}$ was added dropwise a solution of $n$-BuLi (210 mL, $505 \mathrm{mmol}, 2.4 \mathrm{M}$ in hexane) over $30 \mathrm{~min}$. After the solution was stirred at $-78{ }^{\circ} \mathrm{C}$ for $30 \mathrm{~min}$, a solution of HMPA $(87.5 \mathrm{~mL}, 505 \mathrm{mmol})$ in THF $(10 \mathrm{~mL})$ was added and the resulting solution was continued to stirr at $-78{ }^{\circ} \mathrm{C}$ for $10 \mathrm{~min}$. A solution of 12 (20.6 g, $50.5 \mathrm{mmol})$ in THF $(20 \mathrm{~mL})$ was then added slowly. The resulting solution was stirred at $-78{ }^{\circ} \mathrm{C}$ for $30 \mathrm{~min}$, and then warmed to $-40{ }^{\circ} \mathrm{C}$ over a period of $1 \mathrm{~h}$ to result a yellow solution. After the yellow solution was cooled to $-78^{\circ} \mathrm{C}$ again, MeI $(47.5 \mathrm{~mL}, 757 \mathrm{mmol})$ was added. After completed the addition, the reaction mixture was allow to warm to room temperature naturally. Saturated aqueuous $\mathrm{NH}_{4} \mathrm{Cl}$ solution $(100 \mathrm{~mL})$ was added to quench the reaction, and the reaction mixture was extracted with EtOAc $(3 \times 100 \mathrm{~mL})$. The organic phase was dried over anhydrous $\mathrm{Na}_{2} \mathrm{SO}_{4}$, and concentrated in vacuo. The residue was purified by chromatography on silica gel (ethyl acetate/petroleum =1:40) to afford 13 (20.1 $\mathrm{g}, 94 \%$ ) as a colorless oil $\left(R_{\mathrm{f}}=0.52\right.$, ethyl acetate/petroleum $\left.=1: 20\right) .[\alpha]_{\mathrm{D}}^{26}+6.2\left(c 1.0, \mathrm{CHCl}_{3}\right) .{ }^{1} \mathrm{H} \mathrm{NMR}\left(400 \mathrm{MHz}, \mathrm{CDCl}_{3}\right) \delta 6.60(\mathrm{~s}$, $1 \mathrm{H}), 6.48(\mathrm{~s}, 1 \mathrm{H}), 4.44(\mathrm{dd}, J=11.4,6.2 \mathrm{~Hz}, 1 \mathrm{H}), 3.79-3.71(\mathrm{~m}, 4 \mathrm{H}), 3.70-3.63(\mathrm{~m}, 1 \mathrm{H}), 3.42(\mathrm{~d}, J=6.4 \mathrm{~Hz}, 1 \mathrm{H}), 2.86-2.76(\mathrm{~m}$, $1 \mathrm{H}), 2.22(\mathrm{~s}, 3 \mathrm{H}), 2.16(\mathrm{~s}, 3 \mathrm{H}), 2.11-2.00(\mathrm{~m}, 1 \mathrm{H}), 2.00-1.90(\mathrm{~m}, 1 \mathrm{H}), 1.56-1.47(\mathrm{~m}, 1 \mathrm{H}), 1.37(\mathrm{~s}, 3 \mathrm{H}), 0.83(\mathrm{t}, J=7.0 \mathrm{~Hz}, 3 \mathrm{H})$, 0.73 (s, 9H), -0.14 (s, 3H), -0.28 (s, 3H). ${ }^{13} \mathrm{C} \mathrm{NMR}\left(101 \mathrm{MHz}, \mathrm{CDCl}_{3}\right) \delta 176.2(1 \mathrm{C}, \mathrm{OC}=\mathrm{O}), 156.8(1 \mathrm{C}, \mathrm{Ar}-\mathrm{C}), 137.9(1 \mathrm{C}, \mathrm{Ar}-$ 
C), 133.4 (1C, Ar-C), 123.6 (1C, Ar-CH), 122.3 (1C, Ar-C), 109.2 (1C, Ar-CH), $74.9(1 \mathrm{C}, \mathrm{CH}), 59.9\left(1 \mathrm{C}, \mathrm{OCH}_{2}\right), 55.8(1 \mathrm{C}, \mathrm{CH})$, $55.6\left(1 \mathrm{C}, \mathrm{OCH}_{3}\right), 53.7(1 \mathrm{C}, \mathrm{CH}), 34.4\left(1 \mathrm{C}, \mathrm{CH}_{2}\right), 33.6\left(1 \mathrm{C}, \mathrm{CH}_{2}\right), 28.3\left(1 \mathrm{C}, \mathrm{CH}_{3}\right), 25.6\left(3 \mathrm{C}, \mathrm{CH}_{3}\right), 21.5\left(1 \mathrm{C}, \mathrm{CH}_{3}\right), 17.9(1 \mathrm{C}, \mathrm{C})$, $13.5\left(1 \mathrm{C}, \mathrm{CH}_{3}\right), 11.5\left(1 \mathrm{C}, \mathrm{CH}_{3}\right),-5.2\left(1 \mathrm{C}, \mathrm{CH}_{3}\right),-5.3\left(1 \mathrm{C}, \mathrm{CH}_{3}\right)$. IR $(\mathrm{KBr}): v_{\max } 2953,2930,2900,2856,1727,1613,1581,1463$, 1254, 1145, 1126, 1064, 912, 835, 773, $747 \mathrm{~cm}^{-1}$ HRMS (ESI) Calcd for $\mathrm{C}_{24} \mathrm{H}_{41} \mathrm{O}_{4} \mathrm{Si}\left([\mathrm{M}+\mathrm{H}]^{+}\right)$: 421.2769 ; Found: 421.2775 .

\section{Synthesis of cyclopentanone $4 .^{6}$}

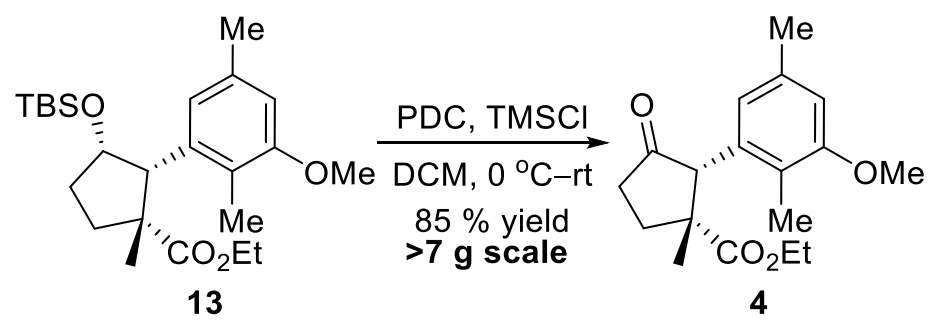

To a suspension of PDC (19.2 g, $50.8 \mathrm{mmol})$ in DCM (50 mL) was added trimethylchlorosilane $(14.9 \mathrm{~mL}, 118.9 \mathrm{mmol})$ at $0{ }^{\circ} \mathrm{C}$, and the dark red-brown mixture was stirred for $30 \mathrm{~min}$. After addition of compound 13 (7.1 g, 16.9 mmol) was added at $0{ }^{\circ} \mathrm{C}$, the reaction mixture was continued to stirr at room temperature over night. After filtration to remove the solid through a pad of silica gel, the resulting dark solution was concentrated in vacuo. The residue was purified by chromatography on silica gel (ethyl acetate/petroleum $=1: 5)$ to afford cyclopantanone $4\left(4.4 \mathrm{~g}, 85 \%\right.$ yield) as a yellow oil $\left(R_{\mathrm{f}}=0.34\right.$, ethyl acetate/petroleum = 1:5). $[\alpha]_{\mathrm{D}}^{27}-121.8\left(c 1.0, \mathrm{CHCl}_{3}\right) .{ }^{1} \mathrm{H} \mathrm{NMR}\left(300 \mathrm{MHz}, \mathrm{CDCl}_{3}\right) \delta 6.57(\mathrm{~s}, 1 \mathrm{H}), 6.27(\mathrm{~s}, 1 \mathrm{H}), 3.93(\mathrm{q}, J=7.1$ $\mathrm{Hz}, 2 \mathrm{H}), 3.78(\mathrm{~s}, 3 \mathrm{H}), 3.68(\mathrm{~s}, 1 \mathrm{H}), 2.85(\mathrm{~m}, 1 \mathrm{H}), 2.67-2.44(\mathrm{~m}, 2 \mathrm{H}), 2.24(\mathrm{~s}, 3 \mathrm{H}), 2.13(\mathrm{~s}, 3 \mathrm{H}), 1.94(\mathrm{~m}, 1 \mathrm{H}), 1.44(\mathrm{~s}, 3 \mathrm{H}), 1.07$ $(\mathrm{t}, J=7.1 \mathrm{~Hz}, 3 \mathrm{H}) .{ }^{13} \mathrm{C} \mathrm{NMR}\left(75 \mathrm{MHz}, \mathrm{CDCl}_{3}\right) \delta 215.6(1 \mathrm{C}, \mathrm{C}=\mathrm{O}), 174.5(1 \mathrm{C}, \mathrm{OC}=\mathrm{O}), 157.2(1 \mathrm{C}, \mathrm{Ar}-\mathrm{C}), 135.1(1 \mathrm{C}, \mathrm{Ar}-\mathrm{C})$, 133.9 (1C, Ar-C), 123.2 (1C, Ar-C), 121.1 (1C, Ar-CH), 110.0 (1C, Ar-CH), $61.6(1 \mathrm{C}, \mathrm{CH}), 60.3\left(1 \mathrm{C}, \mathrm{OCH}_{2}\right), 55.0\left(1 \mathrm{C}, \mathrm{OCH}_{3}\right)$, $52.7(1 \mathrm{C}, \mathrm{C}), 36.7\left(1 \mathrm{C}, \mathrm{CH}_{2}\right), 32.2\left(1 \mathrm{C}, \mathrm{CH}_{2}\right), 23.9\left(1 \mathrm{C}, \mathrm{CH}_{3}\right), 21.2\left(1 \mathrm{C}, \mathrm{CH}_{3}\right), 13.4\left(1 \mathrm{C}, \mathrm{CH}_{3}\right), 11.7\left(1 \mathrm{C}, \mathrm{CH}_{3}\right) . \mathrm{IR}\left({\mathrm{KBr}): v_{\max }}\right.$ 2978, 2938, 1730, 1610, 1580, 1461, 1321, 1281, 1185, 1147, $1023 \mathrm{~cm}^{-1}$ HRMS (ESI) Calcd for $\mathrm{C}_{18} \mathrm{H}_{25} \mathrm{O}_{4}\left([\mathrm{M}+\mathrm{H}]^{+}\right): 305.1747$; Found: 305.1745 .

\section{(B) Synthesis of Dialdehyde 5.}

\section{Synthesis of compound 16 .}

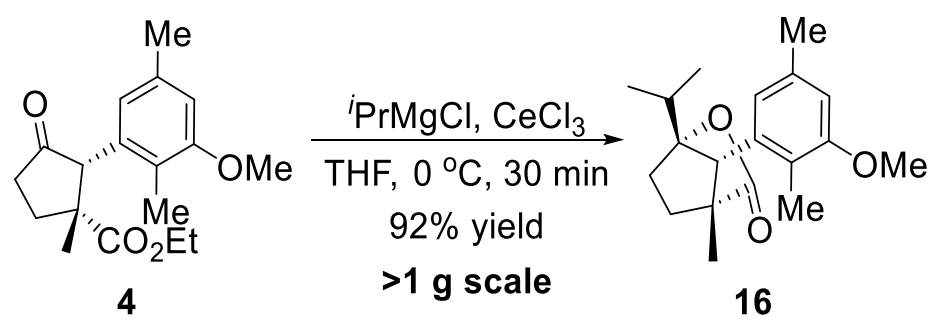

To a solution of compound $4(1.1 \mathrm{~g}, 3.62 \mathrm{mmol})$ in anhydrous THF $(20 \mathrm{~mL})$ was added to a suspension of cerium chloride $(8.9 \mathrm{~g}, 36.2 \mathrm{mmol})$ in anhydrous THF $(20 \mathrm{~mL})$ at $0{ }^{\circ} \mathrm{C}$. The mixture was allowed to well stirr at room temperature for $10 \mathrm{~h}$ and then cooled to $0^{\circ} \mathrm{C}$. A solution of ipropyl isopropylmagnesium chloride $(21.7 \mathrm{~mL}, 43.3 \mathrm{mmol}, 2.0 \mathrm{M}$ in THF) was then added dropwise at $0-10{ }^{\circ} \mathrm{C}$ under vigorous stirring. After stirring for $30 \mathrm{~min}$ at $0{ }^{\circ} \mathrm{C}$, the reaction mixture was quenched carefully with $10 \%$ aqueous acetic $(10 \mathrm{~mL})$ solution, and extracted with EtOAc $(3 \times 10 \mathrm{~mL})$. The combined organic solution was washed with brine, dried over $\mathrm{Na}_{2} \mathrm{SO}_{4}$, and concentrated in vacuo. The residue was subjected to toluene (twice) to remove the water and $\mathrm{AcOH}$, and purified by chromatography on silica gel (ethyl acetate/petroleum $=1: 10$ ) to afford $\mathbf{1 6}(1.0 \mathrm{~g}, 92 \%$ yield) as a white solid $\left(R_{\mathrm{f}}=0.73\right.$, ethyl acetate/petroleum $\left.=1: 5\right) . \mathrm{Mp} 189-191{ }^{\circ} \mathrm{C},[\alpha]_{\mathrm{D}}^{26}+29.6\left(c 1.0, \mathrm{CHCl}_{3}\right) .{ }^{1} \mathrm{H} \mathrm{NMR}\left(400 \mathrm{MHz}, \mathrm{CDCl}_{3}\right) \delta 6.79$ 
$(\mathrm{s}, 1 \mathrm{H}), 6.59(\mathrm{~s}, 1 \mathrm{H}), 3.79(\mathrm{~s}, 3 \mathrm{H}), 3.22(\mathrm{~s}, 1 \mathrm{H}), 2.35-2.22(\mathrm{~m}, 4 \mathrm{H}), 2.10(\mathrm{~s}, 3 \mathrm{H}), 2.08-2.00(\mathrm{~m}, 1 \mathrm{H}), 1.94-1.77(\mathrm{~m}, 3 \mathrm{H}), 1.06(\mathrm{~d}$, $J=6.8 \mathrm{~Hz}, 3 \mathrm{H}), 1.05(\mathrm{~s}, 3 \mathrm{H}), 0.72(\mathrm{~d}, J=6.8 \mathrm{~Hz}, 3 \mathrm{H}) .{ }^{13} \mathrm{C} \mathrm{NMR}\left(101 \mathrm{MHz}, \mathrm{CDCl}_{3}\right) \delta 180.1(1 \mathrm{C}, \mathrm{OC}=\mathrm{O}), 157.4(1 \mathrm{C}, \mathrm{Ar}-\mathrm{C})$, 136.3 (1C, Ar-C), 133.6 (1C, Ar-C), 122.7 (1C, Ar-C), 119.4 (1C, Ar-CH), 110.2 (1C, Ar-CH), 96.9 (1C, C), 56.7 (1C, CH), 55.3 $\left(1 \mathrm{C}, \mathrm{OCH}_{3}\right), 54.1(1 \mathrm{C}, \mathrm{C}), 31.0\left(1 \mathrm{C}, \mathrm{CH}_{2}\right), 28.0(1 \mathrm{C}, \mathrm{CH}), 27.7\left(1 \mathrm{C}, \mathrm{CH}_{2}\right), 21.8\left(1 \mathrm{C}, \mathrm{CH}_{3}\right), 17.4\left(1 \mathrm{C}, \mathrm{CH}_{3}\right), 16.7\left(1 \mathrm{C}, \mathrm{CH}_{3}\right), 12.7$ $\left(1 \mathrm{C}, \mathrm{CH}_{3}\right), 11.4\left(1 \mathrm{C}, \mathrm{CH}_{3}\right)$. IR (KBr): $v_{\max } 2965,2938,2873,1762,1611,1581,1467,1303,1208,1168,1144,1101,1041,905$, $842 \mathrm{~cm}^{-1}$ HRMS (ESI) Calcd for $\mathrm{C}_{19} \mathrm{H}_{27} \mathrm{O}_{3}\left([\mathrm{M}+\mathrm{H}]^{+}\right)$: 303.1955; Found: 303.1954 .

The complex $16(20 \mathrm{mg})$ was dissolved in mixture solvents of DCM (1 mL) and hexane $(1 \mathrm{~mL})$. After slowly evaporation of solvents at ambient temperautre, the fine crystals which were suitable for the X-ray diffraction analyses were obtained.

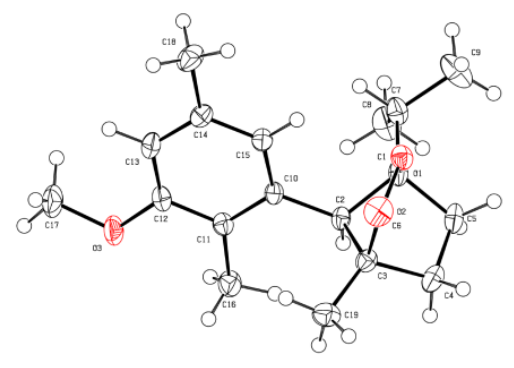

Table S2. Crystal data and structure refinement for (+)-16

\begin{tabular}{|c|c|}
\hline Empirical formula & $\mathrm{C}_{19} \mathrm{H}_{26} \mathrm{O}_{3}$ \\
\hline Formula weight & 302.40 \\
\hline Temperature & $173.15 \mathrm{~K}$ \\
\hline Wavelength & $1.54184 \mathrm{~A}$ \\
\hline Crystal system, space group & Orthorhombic, $\quad \mathrm{P} 2(1) 2(1) 2(1)$ \\
\hline Unit cell dimensions & $\begin{array}{l}a=6.43415(9) \text { A } \quad \text { alpha }=90 \mathrm{deg} . \\
\mathrm{b}=13.96950(20) \mathrm{A} \quad \text { beta }=90 \mathrm{deg} . \\
\mathrm{c}=18.8234(2) \mathrm{A} \quad \text { gamma }=90 \mathrm{deg} .\end{array}$ \\
\hline Volume & $1691.88(4) \mathrm{A}^{\wedge} 3$ \\
\hline Z, Calculated density & $4, \quad 1.187 \mathrm{Mg} / \mathrm{m}^{\wedge} 3$ \\
\hline Absorption coefficient & $0.623 \mathrm{~mm}^{\wedge}-1$ \\
\hline $\mathrm{F}(000)$ & 656 \\
\hline Crystal size & $0.32 \times 0.26 \times 0.12 \mathrm{~mm}$ \\
\hline Theta range for data collection & 3.941 to $78.838 \mathrm{deg}$. \\
\hline Limiting indices & $-7<=\mathrm{h}<=6,-17<=\mathrm{k}<=17,-23<=\mathrm{l}<=22$ \\
\hline Reflections collected / unique & $13103 / 3566[\mathrm{R}(\mathrm{int})=0.0205]$ \\
\hline Completeness to theta $=67.684$ & $100.0 \%$ \\
\hline Absorption correction & Semi-empirical from equivalents \\
\hline Max. and min. transmission & 1.00000 and 0.84635 \\
\hline Refinement method & Full-matrix least-squares on $\mathrm{F}^{\wedge} 2$ \\
\hline Data / restraints / parameters & $3566 / 0 / 206$ \\
\hline Goodness-of-fit on $\mathrm{F}^{\wedge} 2$ & 1.041 \\
\hline Final $R$ indices $[\mathrm{I}>2 \operatorname{sigma}(\mathrm{I})]$ & $\mathrm{R} 1=0.0321, \mathrm{wR} 2=0.0848$ \\
\hline $\mathrm{R}$ indices (all data) & $\mathrm{R} 1=0.0322, \mathrm{wR} 2=0.0850$ \\
\hline Absolute structure parameter & $-0.02(4)$ \\
\hline Extinction coefficient & $0.0129(9)$ \\
\hline Flack parameter & $-0.005(198)$ \\
\hline Largest diff. peak and hole & 0.199 and -0.216 e. $A^{\wedge}-3$ \\
\hline
\end{tabular}




\section{Synthesis of compound 19.}

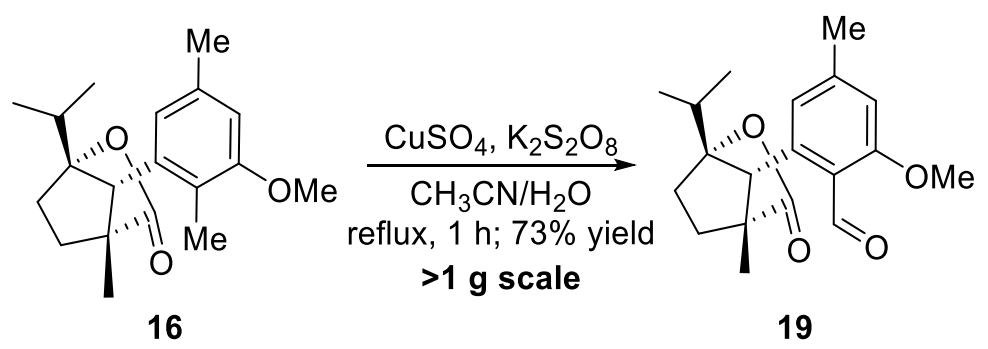

To a solution of compound $\mathbf{1 6}(1.67 \mathrm{~g}, 5.53 \mathrm{mmol})$ in acetonitrile/water $(1: 1)(200 \mathrm{~mL})$ was added cooper sulfate $(880 \mathrm{mg}$, $5.53 \mathrm{mmol})$, and potassium peroxydisulfate $(4.48 \mathrm{~g}, 16.5 \mathrm{mmol})$, and the resulting reaction mixture was heated to reflux under vigorously stirring for $1 \mathrm{~h}$ to complete the reaction. The mixture was cooled to room temperature and diluted with DCM $(50 \mathrm{~mL})$. After seperation of the organic layer, the aqueous layer was extracted with DCM $(3 \times 20 \mathrm{~mL})$. The combined extracts were washed with brine and dried over $\mathrm{Na}_{2} \mathrm{SO}_{4}$ and concentrated in vacuo. The residue was purified by chromatography on silica gel (ethyl acetate/petroleum $=1: 5)$ to afford $\mathbf{1 9}\left(1.3 \mathrm{~g}, 73 \%\right.$ yield) as a yellow oil $\left(R_{\mathrm{f}}=0.36\right.$, ethyl acetate/petroleum $\left.=1: 5\right)$. $[\alpha]_{\mathrm{D}}^{26}$ $-2.6\left(c\right.$ 1.0, $\left.\mathrm{CHCl}_{3}\right) .{ }^{1} \mathrm{H}$ NMR (400 MHz, $\left.\mathrm{CDCl}_{3}\right) \delta 10.55(\mathrm{~s}, 1 \mathrm{H}), 6.95(\mathrm{~s}, 1 \mathrm{H}), 6.72(\mathrm{~s}, 1 \mathrm{H}), 4.56(\mathrm{~s}, 1 \mathrm{H}), 3.89(\mathrm{~s}, 3 \mathrm{H}), 2.36(\mathrm{~s}$, $3 \mathrm{H}), 2.33-2.18(\mathrm{~m}, 1 \mathrm{H}), 2.11-1.95(\mathrm{~m}, 2 \mathrm{H}), 1.87-1.72(\mathrm{~m}, 2 \mathrm{H}), 1.06(\mathrm{~d}, J=6.8 \mathrm{~Hz}, 3 \mathrm{H}), 1.02(\mathrm{~s}, 3 \mathrm{H}), 0.72(\mathrm{~d}, J=6.8 \mathrm{~Hz}, 3 \mathrm{H})$. ${ }^{13} \mathrm{C}$ NMR (101 MHz, $\left.\mathrm{CDCl}_{3}\right) \delta 192.7$ (1C, CHO), 180.7 (1C, OC=O), 163.9 (1C, Ar-C), 146.6 (1C, Ar-C), 138.2 (1C, Ar-C), 121.9 (1C, Ar-C), 121.1 (1C, Ar-CH), $111.2(1 \mathrm{C}, \mathrm{Ar}-\mathrm{CH}), 97.1(1 \mathrm{C}, \mathrm{C}), 55.7\left(1 \mathrm{C}, \mathrm{CH}_{3}\right), 54.9(1 \mathrm{C}, \mathrm{C}), 54.1(1 \mathrm{C}, \mathrm{CH}), 30.7(1 \mathrm{C}$, $\left.\mathrm{CH}_{2}\right), 28.1(1 \mathrm{C}, \mathrm{CH}), 27.9\left(1 \mathrm{C}, \mathrm{CH}_{2}\right), 22.7\left(1 \mathrm{C}, \mathrm{CH}_{3}\right), 17.5\left(1 \mathrm{C}, \mathrm{CH}_{3}\right), 16.8\left(1 \mathrm{C}, \mathrm{CH}_{3}\right), 12.3\left(1 \mathrm{C}, \mathrm{CH}_{3}\right) . \mathrm{IR}(\mathrm{KBr}): v_{\max } 3011$, 2966, 2876, 1774, 1680, 1604, 1570, 1465,1413,1304, 1280, 1197, 1164, 1092, 1040, 907, $753 \mathrm{~cm}^{-1}$ HRMS (ESI) Calcd for $\mathrm{C}_{19} \mathrm{H}_{25} \mathrm{O}_{4}\left([\mathrm{M}+\mathrm{H}]^{+}\right): 317.1747$; Found: 317.1751 .

\section{Synthesis of compound 20.}

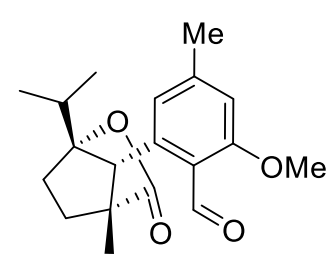

19

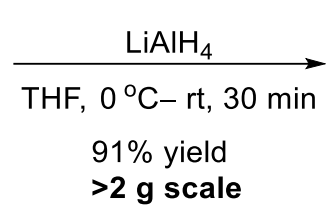

$>2$ g scale

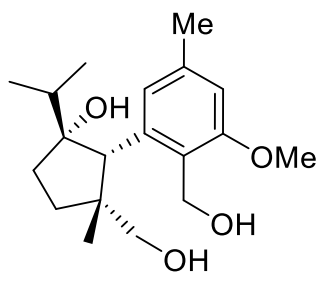

20

To a solution of compound $19(2.78 \mathrm{~g}, 8.8 \mathrm{mmol})$ in THF $(40 \mathrm{~mL})$ was added $\mathrm{LiAlH}_{4}(1.67 \mathrm{~g}, 44.0 \mathrm{mmol})$ at $0{ }^{\circ} \mathrm{C}$, and reaction mixture was stirred for $30 \mathrm{~min}$ at room temperature. EtOAc $(20 \mathrm{~mL})$ was then added to quench the reaction. After the reaction mixture was stirred for additional $1 \mathrm{~h}$, saturated aqueous potassium sodium tartrate solution $(10 \mathrm{~mL})$ was added. The mixture was continued to stirr for $30 \mathrm{~min}$, the organic layer was separated and the aqueous layer was extracted with EtOAc $(3 \times$ $10 \mathrm{~mL}$ ). The combined extracts were washed with brine, dried over $\mathrm{Na}_{2} \mathrm{SO}_{4}$, and concentrated in vacuo. The residue was purified by chromatography on silica gel (ethyl acetate/petroleum $=1: 2)$ to afford $\mathbf{2 0}\left(2.58 \mathrm{~g}, 91 \%\right.$ yield) as a white solid $\left(R_{\mathrm{f}}=0.22\right.$, ethyl acetate/petroleum = 1:3). Mp: $155-156{ }^{\circ} \mathrm{C},[\alpha]_{\mathrm{D}}^{27}+8.8\left(c 1.0, \mathrm{CHCl}_{3}\right) .{ }^{1} \mathrm{H}$ NMR $\left(400 \mathrm{MHz}, \mathrm{CDCl}_{3}\right) \delta 7.25(\mathrm{~s}, 1 \mathrm{H}), 6.66(\mathrm{~s}, 1 \mathrm{H})$, $4.81-4.65(\mathrm{~m}, 2 \mathrm{H}), 3.87$ (s, 3H), $3.30(\mathrm{~d}, J=11.2 \mathrm{~Hz}, 1 \mathrm{H}), 3.22(\mathrm{~s}, 1 \mathrm{H}), 3.22(\mathrm{~d}, J=10.8 \mathrm{~Hz}, 1 \mathrm{H}), 2.93(\mathrm{~s}, 1 \mathrm{H}), 2.58(\mathrm{t}, J=6.8$ $\mathrm{Hz}, 1 \mathrm{H}), 2.36(\mathrm{~s}, 3 \mathrm{H}), 2.20(\mathrm{dt}, J=13.2,8.8 \mathrm{~Hz}, 1 \mathrm{H}), 1.97(\mathrm{dt}, J=13.6,9.6 \mathrm{~Hz}, 1 \mathrm{H}), 1.76-1.62(\mathrm{~m}, 2 \mathrm{H}), 1.60(\mathrm{~s}, 1 \mathrm{H}), 1.58-1.50$ (m, 1H), $0.97(\mathrm{~s}, 3 \mathrm{H}), 0.87(\mathrm{~d}, J=6.8 \mathrm{~Hz}, 3 \mathrm{H}), 0.79(\mathrm{~d}, J=6.8 \mathrm{~Hz}, 3 \mathrm{H}) .{ }^{13} \mathrm{C} \mathrm{NMR}\left(101 \mathrm{MHz}, \mathrm{CDCl}_{3}\right) \delta 158.2(1 \mathrm{C}, \mathrm{Ar}-\mathrm{C}), 137.8$ (1C, Ar-C), 136.9 (1C, Ar-C), 126.4 (1C, Ar-C), 123.3 (1C, Ar-CH), 109.6 (1C, Ar-CH), $86.8(1 \mathrm{C}, \mathrm{C}), 67.7$ (1C, $\left.\mathrm{CH}_{2}\right), 57.3(1 \mathrm{C}$, $\left.\mathrm{CH}_{2}\right), 55.3\left(1 \mathrm{C}, \mathrm{OCH}_{3}\right), 55.0(1 \mathrm{C}, \mathrm{CH}), 47.6(1 \mathrm{C}, \mathrm{C}), 35.8(1 \mathrm{C}, \mathrm{CH}), 34.7\left(1 \mathrm{C}, \mathrm{CH}_{2}\right), 32.6\left(1 \mathrm{C}, \mathrm{CH}_{2}\right), 26.2\left(1 \mathrm{C}, \mathrm{CH}_{3}\right), 22.2(1 \mathrm{C}$, 
$\left.\mathrm{CH}_{3}\right), 18.4\left(1 \mathrm{C}, \mathrm{CH}_{3}\right), 17.8\left(1 \mathrm{C}, \mathrm{CH}_{3}\right) . \mathrm{IR}(\mathrm{KBr}): v_{\max } 3596,3434,3229,2952,2871,1609,1575,1465,1293,1190,1177,1139$, 1097, 1042, 1004, $945 \mathrm{~cm}^{-1} \mathrm{HRMS}$ (ESI) Calcd for $\mathrm{C}_{19} \mathrm{H}_{30} \mathrm{NaO}_{4}\left([\mathrm{M}+\mathrm{Na}]^{+}\right)$: 345.2036; Found: 345.2036.

\section{Synthesis of compound 21.}

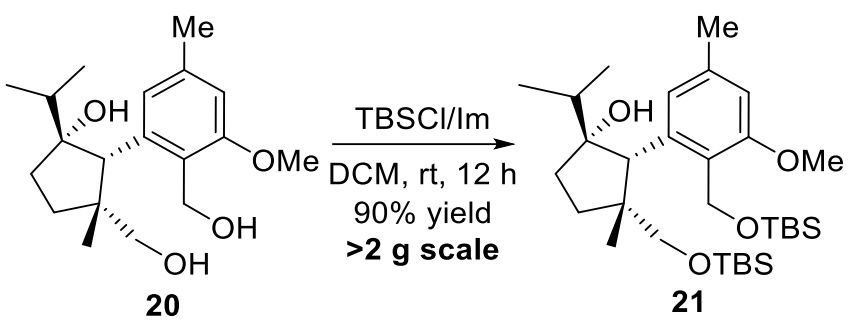

To a solution of compound 20 (2.42 g, $7.5 \mathrm{mmol})$ and imidazole (3.12 g, $45.9 \mathrm{mmol})$ in DCM (50 mL) was added TBSCl $(3.43 \mathrm{~g}, 22.9 \mathrm{mmol})$, and the resulting reaction mixture was stirred at room temperature for $12 \mathrm{~h}$. The suspension was filtered and concentrated in vacuo. The residue was purified by chromatography on silica gel (ethyl acetate/petroleum $=1: 5$ ) to afford $21(3.7 \mathrm{~g}, 90 \%$ yield $)$ as a white solid $\left(R_{\mathrm{f}}=0.8\right.$, ethyl acetate/petroleum $\left.=1: 5\right) . \mathrm{Mp}: 71-72{ }^{\circ} \mathrm{C}[\alpha]_{\mathrm{D}}^{28}-2.4\left(c 1.0, \mathrm{CHCl}_{3}\right) .{ }^{1} \mathrm{H} \mathrm{NMR}$ $\left(400 \mathrm{MHz}_{\mathrm{CDCl}}\right) \delta 7.30(\mathrm{~s}, 1 \mathrm{H}), 6.59(\mathrm{~s}, 1 \mathrm{H}), 4.73(\mathrm{~s}, 2 \mathrm{H}), 4.67(\mathrm{~s}, 1 \mathrm{H}), 3.79(\mathrm{~s}, 3 \mathrm{H}), 3.29(\mathrm{~d}, J=10.2 \mathrm{~Hz}, 1 \mathrm{H}), 3.23(\mathrm{~s}, 1 \mathrm{H})$, $3.04(\mathrm{~d}, J=10.2 \mathrm{~Hz}, 1 \mathrm{H}), 2.32(\mathrm{~s}, 3 \mathrm{H}), 2.14(\mathrm{dt}, J=13.2,9.2 \mathrm{~Hz}, 1 \mathrm{H}), 1.90(\mathrm{dt}, J=12.8,10 \mathrm{~Hz}, 1 \mathrm{H}), 1.74-1.61(\mathrm{~m}, 2 \mathrm{H}), 1.50$ $(\mathrm{ddd}, J=13.2,10.4,3.0 \mathrm{~Hz}, 1 \mathrm{H}), 0.97(\mathrm{~s}, 9 \mathrm{H}), 0.94(\mathrm{~s}, 3 \mathrm{H}), 0.90(\mathrm{~s}, 9 \mathrm{H}), 0.84$ (d, $J=6.8 \mathrm{~Hz}, 3 \mathrm{H}), 0.73(\mathrm{~d}, J=6.8 \mathrm{~Hz}, 3 \mathrm{H}), 0.13$ $(\mathrm{d}, J=8.3 \mathrm{~Hz}, 6 \mathrm{H}), 0.09$ (d, $J=7.1 \mathrm{~Hz}, 6 \mathrm{H}) .{ }^{13} \mathrm{C} \mathrm{NMR}\left(101 \mathrm{MHz}, \mathrm{CDCl}_{3}\right) \delta 157.9(1 \mathrm{C}, \mathrm{Ar}-\mathrm{C}), 138.7$ (1C, Ar-C), 136.8 (1C, ArC), 126.0 (1C, Ar-C), 124.1 (1C, Ar-CH), 109.5 (1C, Ar-CH), 85.7 (1C, C), $68.6\left(1 \mathrm{C}, \mathrm{CH}_{2}\right), 56.1\left(1 \mathrm{C}, \mathrm{CH}_{2}\right), 55.6(1 \mathrm{C}, \mathrm{CH}), 55.0$ $\left(1 \mathrm{C}, \mathrm{OCH}_{3}\right), 47.4(1 \mathrm{C}, \mathrm{C}), 36.0\left(1 \mathrm{C}, \mathrm{CH}_{2}\right), 35.3(1 \mathrm{C}, \mathrm{CH}), 31.9\left(1 \mathrm{C}, \mathrm{CH}_{2}\right), 26.9\left(1 \mathrm{C}, \mathrm{CH}_{3}\right), 26.1\left(6 \mathrm{C}, \mathrm{CH}_{3}\right), 22.2\left(1 \mathrm{C}, \mathrm{CH}_{3}\right), 18.8$ $\left(1 \mathrm{C}, \mathrm{CH}_{3}\right), 18.6(1 \mathrm{C}, \mathrm{C}), 18.5(1 \mathrm{C}, \mathrm{C}), 18.3\left(1 \mathrm{C}, \mathrm{CH}_{3}\right),-5.3\left(1 \mathrm{C}, \mathrm{CH}_{3}\right),-5.4\left(1 \mathrm{C}, \mathrm{CH}_{3}\right),-5.4\left(1 \mathrm{C}, \mathrm{CH}_{3}\right),-5.6\left(1 \mathrm{C}, \mathrm{CH}_{3}\right) . \mathrm{IR}$ $(\mathrm{KBr}): v_{\max } 3393,2954,2930,2882,2856,1612,1578,1466,1295,1255,1147,1097,1059,1030,1005,838,787,776 \mathrm{~cm}^{-1}$ HRMS (ESI) Calcd for $\mathrm{C}_{31} \mathrm{H}_{58} \mathrm{NaO}_{4} \mathrm{Si}_{2}\left([\mathrm{M}+\mathrm{Na}]^{+}\right)$: 573.3766 ; Found: 573.3776.

Note: Compound 21 could be synthesized in 57\% yield from ketone 4 without purification of the intermediates (55\% overall yield for every step purified by column chromatography).

\section{Synthesis of compounds 22a and 22b.}

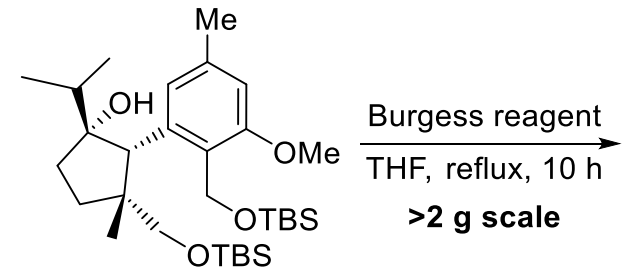

21
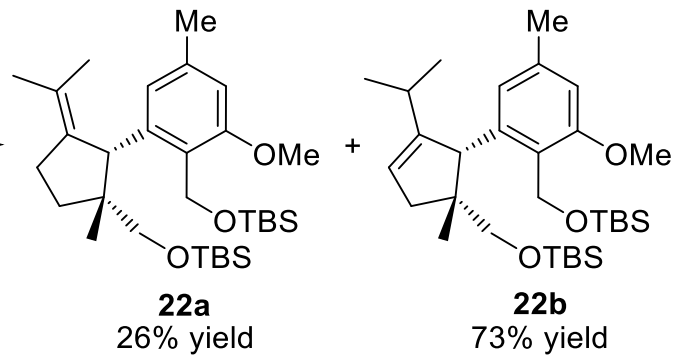

To a solution of compound $21(2.2 \mathrm{~g}, 4.0 \mathrm{mmol})$ in THF $(50 \mathrm{~mL})$ was added Burgess reagent $(4.8 \mathrm{~g}, 20 \mathrm{mmol})$ at room temperature under argon atmosphere, and the reaction mixture was heated at reflux for $10 \mathrm{~h}$. After cooled to room temparature, the reaction was quenched with $\mathrm{H}_{2} \mathrm{O}(10 \mathrm{~mL})$, and extracted with EtOAc $(3 \times 10 \mathrm{~mL})$. The combined extracts were washed with brine, dried over $\mathrm{Na}_{2} \mathrm{SO}_{4}$, and concentrated in vacuo. The residue was fitered through a short silica column to afford a mixture of 22a and 22b (total $2.1 \mathrm{~g}, 99 \%$ yield). The ${ }^{1} \mathrm{H}$ NMR of the mixture showed that the yield of 22a is $26 \%$ and the yield of $22 \mathbf{b}$ is 73\%. The pure 22a and $\mathbf{2 2 b}$ were obtained by three times chromatography on silica gel (ethyl acetate/petroleum $=1: 20$ ) For 22a: $\left(R_{\mathrm{f}}=0.90\right.$, ethyl acetate/petroleum $\left.=1: 5\right) .[\alpha]_{\mathrm{D}}^{26}+28.3\left(c 0.1, \mathrm{CHCl}_{3}\right){ }^{1} \mathrm{H} \mathrm{NMR}\left(400 \mathrm{MHz}, \mathrm{CDCl}_{3}\right) \delta 6.47(\mathrm{~s}, 1 \mathrm{H}), 6.26(\mathrm{~s}$, $1 \mathrm{H}), 4.86-4.76(\mathrm{~m}, 2 \mathrm{H}), 3.88(\mathrm{~s}, 1 \mathrm{H}), 3.77(\mathrm{~s}, 3 \mathrm{H}), 2.93-2.85(\mathrm{~m}, 2 \mathrm{H}), 2.53-2.43(\mathrm{~m}, 2 \mathrm{H}), 2.25(\mathrm{~s}, 3 \mathrm{H}), 1.79-1.65(\mathrm{~m}, 1 \mathrm{H}), 1.62$ $(\mathrm{s}, 3 \mathrm{H}), 1.47-1.36(\mathrm{~m}, 4 \mathrm{H}), 1.08(\mathrm{~s}, 3 \mathrm{H}), 0.90(\mathrm{~s}, 9 \mathrm{H}), 0.78(\mathrm{~s}, 9 \mathrm{H}), 0.09(\mathrm{~s}, 3 \mathrm{H}),-0.00(\mathrm{~s}, 3 \mathrm{H}),-0.14(\mathrm{~s}, 3 \mathrm{H}),-0.15(\mathrm{~s}, 3 \mathrm{H}) .{ }^{13} \mathrm{C}$ 
NMR (101 MHz, CDCl 3 ) $\delta 159.4$ (1C, Ar-C), 147.0 (1C, Ar-C), 142.0 (1C, Ar-C), 139.4 (1C, Ar-C), 127.1 (1C, C), 126.6 (1C, C), $123.4(1 \mathrm{C}, \mathrm{Ar}-\mathrm{CH}), 111.2(1 \mathrm{C}, \mathrm{Ar}-\mathrm{CH}), 71.0\left(1 \mathrm{C}, \mathrm{CH}_{2}\right), 58.6\left(1 \mathrm{C}, \mathrm{CH}_{2}\right), 57.5(1 \mathrm{C}, \mathrm{CH}), 54.3\left(1 \mathrm{C}, \mathrm{CH}_{3}\right), 51.1(1 \mathrm{C}, \mathrm{C}), 36.2$ $\left(1 \mathrm{C}, \mathrm{CH}_{2}\right), 30.9\left(1 \mathrm{C}, \mathrm{CH}_{2}\right), 28.3\left(3 \mathrm{C}, \mathrm{CH}_{3}\right), 28.1\left(3 \mathrm{C}, \mathrm{CH}_{3}\right), 26.7\left(1 \mathrm{C}, \mathrm{CH}_{3}\right), 24.2\left(1 \mathrm{C}, \mathrm{CH}_{3}\right), 23.6\left(2 \mathrm{C}, \mathrm{CH}_{3}\right), 20.8(1 \mathrm{C}, \mathrm{C}), 20.5$ (1C, C), $2.1\left(1 \mathrm{C}, \mathrm{CH}_{3}\right),-3.1\left(1 \mathrm{C}, \mathrm{CH}_{3}\right),-3.4\left(1 \mathrm{C}, \mathrm{CH}_{3}\right),-3.5\left(1 \mathrm{C}, \mathrm{CH}_{3}\right)$. IR (KBr): v $\max 2956,2928,2855,1464,1261,1091,1019$, 836, 801, $743 \mathrm{~cm}^{-1}$ HRMS (ESI) Calcd for $\mathrm{C}_{31} \mathrm{H}_{56} \mathrm{NaO}_{3} \mathrm{Si}_{2}\left([\mathrm{M}+\mathrm{Na}]^{+}\right)$: 555.3660; Found: 555.3660. For 22b: $\left(R_{\mathrm{f}}=0.94\right.$, ethyl acetate/petroleum = 1:5). $[\alpha]_{\mathrm{D}}^{28}+33.0\left(\mathrm{c} 1.0, \mathrm{CHCl}_{3}\right) .{ }^{1} \mathrm{H} \mathrm{NMR}\left(400 \mathrm{MHz}, \mathrm{CDCl}_{3}\right) \delta 6.49(\mathrm{~s}, 1 \mathrm{H}), 6.36(\mathrm{~s}, 1 \mathrm{H}), 5.47(\mathrm{~s}, 1 \mathrm{H}), 4.83$ $(\mathrm{d}, J=10.6 \mathrm{~Hz}, 1 \mathrm{H}), 4.71(\mathrm{~d}, J=10.6 \mathrm{~Hz}, 1 \mathrm{H}), 3.85(\mathrm{~s}, 1 \mathrm{H}), 3.77(\mathrm{~s}, 3 \mathrm{H}), 3.03(\mathrm{~d}, J=9.6 \mathrm{~Hz}, 1 \mathrm{H}), 2.98(\mathrm{~d}, J=9.6 \mathrm{~Hz}, 1 \mathrm{H})$, 2.28-2.20 (m, 4H), 2.00-1.87 (m, 2H), $1.20(\mathrm{~s}, 3 \mathrm{H}), 1.00$ (d, $J=6.8 \mathrm{~Hz}, 3 \mathrm{H}), 0.94(\mathrm{~d}, J=6.8 \mathrm{~Hz}, 3 \mathrm{H}), 0.92(\mathrm{~s}, 9 \mathrm{H}), 0.75(\mathrm{~s}, 9 \mathrm{H})$, $0.10(\mathrm{~s}, 3 \mathrm{H}), 0.08$ (s, 3H), -0.19 (s, 3H), $-0.23(\mathrm{~s}, 3 \mathrm{H}) .{ }^{13} \mathrm{C}$ NMR $\left(101 \mathrm{MHz}, \mathrm{CDCl}_{3}\right) \delta 157.6(1 \mathrm{C}, \mathrm{C}), 154.0(1 \mathrm{C}, \mathrm{C}), 141.6(1 \mathrm{C}$, C), $137.2(1 \mathrm{C}, \mathrm{C}), 125.4(1 \mathrm{C}, \mathrm{C}), 121.0(1 \mathrm{C}, \mathrm{CH}), 120.0(1 \mathrm{C}, \mathrm{CH}), 109.3(1 \mathrm{C}, \mathrm{CH}), 68.7\left(1 \mathrm{C}, \mathrm{CH}_{2}\right), 56.3\left(1 \mathrm{C}, \mathrm{CH}_{2}\right), 55.9(1 \mathrm{C}$, $\mathrm{CH}), 55.3\left(1 \mathrm{C}, \mathrm{CH}_{3}\right), 47.7(1 \mathrm{C}, \mathrm{C}), 42.8\left(1 \mathrm{C}, \mathrm{CH}_{3}\right), 28.1(1 \mathrm{C}, \mathrm{CH}), 26.9\left(1 \mathrm{C}, \mathrm{CH}_{3}\right), 26.2\left(3 \mathrm{C}, \mathrm{CH}_{3}\right), 25.9\left(3 \mathrm{C}, \mathrm{CH}_{3}\right), 22.0(1 \mathrm{C}$, $\left.\mathrm{CH}_{3}\right), 21.9\left(1 \mathrm{C}, \mathrm{CH}_{3}\right), 21.4\left(1 \mathrm{C}, \mathrm{CH}_{3}\right), 18.7(1 \mathrm{C}, \mathrm{C}), 18.3(1 \mathrm{C}, \mathrm{C}),-5.2\left(1 \mathrm{C}, \mathrm{CH}_{3}\right),-5.3\left(1 \mathrm{C}, \mathrm{CH}_{3}\right),-5.8\left(1 \mathrm{C}, \mathrm{CH}_{3}\right),-5.8(1 \mathrm{C}$, $\mathrm{CH}_{3}$ ). IR (KBr): $v_{\max } 2955,2928,2896,2856,1610,1580,1463,1290,1254,1147,1087,1057,1019,1006,856,835,814,774$ $\mathrm{cm}^{-1}$ HRMS (ESI) Calcd for $\mathrm{C}_{31} \mathrm{H}_{56} \mathrm{NaO}_{3} \mathrm{Si}_{2}\left([\mathrm{M}+\mathrm{Na}]^{+}\right)$: 555.3660; Found: 555.3665.

\section{Synthesis of compound 23 .}
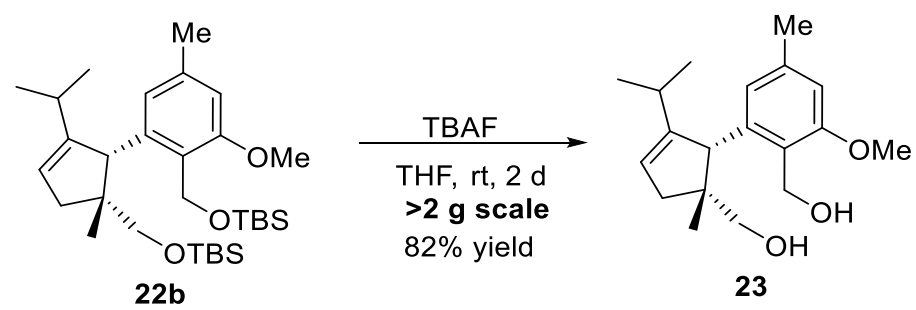

To a solution of the mixture 22a and 22b which contains $2.2 \mathrm{~g}(4.1 \mathrm{mmol}) \mathbf{2 2 b}$ in THF $(50 \mathrm{~mL})$ was added TBAF $(10 \mathrm{~g}$, $38.3 \mathrm{mmol}$ ) at room temperature under argon atmosphere. The reaction mixture was stirred at room temperature for $2 \mathrm{~d}$, saturated aqueous $\mathrm{NH}_{4} \mathrm{Cl}(20 \mathrm{~mL})$ was then added. The mixture was extracted with EtOAc $(3 \times 15 \mathrm{~mL})$, and the combined extracts were washed with brine, dried over $\mathrm{Na}_{2} \mathrm{SO}_{4}$, and concentrated in vacuo. The residue was purified by chromatography on silica gel (ethyl acetate/petroleum $=1: 3)$ to afford $23\left(1.02 \mathrm{~g}, 82 \%\right.$ yield) as a colorless solid $\left(R_{\mathrm{f}}=0.26\right.$, ethyl acetate/petroleum $\left.=1: 5\right)$. $\mathrm{Mp} \mathrm{105-108}{ }^{\circ} \mathrm{C},[\alpha]_{\mathrm{D}}^{27}+163.4\left(\mathrm{c} 1.0, \mathrm{CHCl}_{3}\right) .{ }^{1} \mathrm{H} \mathrm{NMR}\left(400 \mathrm{MHz}, \mathrm{CDCl}_{3}\right) \delta 6.56(\mathrm{~s}, 1 \mathrm{H}), 6.41(\mathrm{~s}, 1 \mathrm{H}), 5.49(\mathrm{~s}, 1 \mathrm{H}), 4.95(\mathrm{~d}, J=$ $11.6 \mathrm{~Hz}, 1 \mathrm{H}), 4.65(\mathrm{~d}, J=11.6 \mathrm{~Hz}, 1 \mathrm{H}), 3.82(\mathrm{~s}, 3 \mathrm{H}), 3.75(\mathrm{~s}, 1 \mathrm{H}), 3.23(\mathrm{~d}, J=11.0 \mathrm{~Hz}, 1 \mathrm{H}), 3.12(\mathrm{~d}, J=11.0 \mathrm{~Hz}, 1 \mathrm{H}), 3.30$ $3.06(\mathrm{br}, 2 \mathrm{H}), 2.28(\mathrm{~s}, 3 \mathrm{H}), 2.23(\mathrm{~d}, J=14.6 \mathrm{~Hz}, 1 \mathrm{H}), 1.96-1.84(\mathrm{~m}, 2 \mathrm{H}), 1.27(\mathrm{~s}, 3 \mathrm{H}), 1.00(\mathrm{~d}, J=6.8 \mathrm{~Hz}, 3 \mathrm{H}), 0.90(\mathrm{~d}, J=6.8$ $\mathrm{Hz}, 3 \mathrm{H}) .{ }^{13} \mathrm{C}$ NMR (101 MHz, CDCl $) \delta 157.9$ (1C, C), 153.8 (1C, C), 140.7 (1C, C), $138.3(1 \mathrm{C}, \mathrm{C}), 124.4(1 \mathrm{C}, \mathrm{C}), 121.0(1 \mathrm{C}$, $\mathrm{CH}), 120.2(1 \mathrm{C}, \mathrm{CH}), 109.4(1 \mathrm{C}, \mathrm{CH}), 67.3\left(1 \mathrm{C}, \mathrm{CH}_{2}\right), 55.6\left(1 \mathrm{C}, \mathrm{CH}_{2}\right), 55.5\left(1 \mathrm{C}, \mathrm{CH}_{3}\right), 55.3(1 \mathrm{C}, \mathrm{CH}), 48.1(1 \mathrm{C}, \mathrm{C}), 42.3(1 \mathrm{C}$, $\left.\mathrm{CH}_{2}\right), 28.3(1 \mathrm{C}, \mathrm{CH}), 26.4\left(1 \mathrm{C}, \mathrm{CH}_{3}\right), 22.0\left(1 \mathrm{C}, \mathrm{CH}_{3}\right), 21.8\left(1 \mathrm{C}, \mathrm{CH}_{3}\right), 21.3\left(1 \mathrm{C}, \mathrm{CH}_{3}\right)$. IR $(\mathrm{KBr}): v_{\max }$ 3278, 2956, 2924, 2869, 2835, 1609, 1578, 1461, 1418, 1287, 1144, 1088, 1040, $1001 \mathrm{~cm}^{-1}$ HRMS (ESI) Calcd for $\mathrm{C}_{19} \mathrm{H}_{28} \mathrm{NaO}_{3}\left([\mathrm{M}+\mathrm{Na}]^{+}\right): 327.1931$; Found: 327.1929 .

\section{Synthesis of compound 5.}

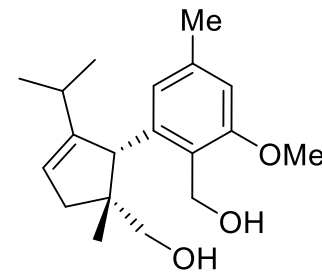

23

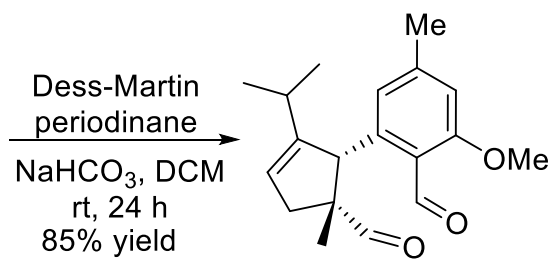

5 
To a solution of Dess-Martin periodinane (7.0 g, $16.4 \mathrm{mmol})$ and $\mathrm{NaHCO}_{3}(4.1 \mathrm{~g}, 49.5 \mathrm{mmol})$ in $\mathrm{CH}_{2} \mathrm{Cl}_{2}(20 \mathrm{~mL})$ was added compound 23 (1.0 g, $3.3 \mathrm{mmol})$ at room temperature under argon atmosphere. The reaction mxiture was stirred at room temperature for $1 \mathrm{~d}$, saturated aqueous $\mathrm{NaHCO}_{3}(10 \mathrm{~mL})$ was then added. The resulting mixture was extracted with $\mathrm{CH}_{2} \mathrm{Cl}_{2}(3 \times$ $10 \mathrm{~mL}$ ), and the combined extracts were washed with brine, dried over $\mathrm{Na}_{2} \mathrm{SO}_{4}$, and concentrated in vacuo. The residue was purified by chromatography on silica gel (ethyl acetate/petroleum $=1: 5)$ to afford $5\left(840 \mathrm{mg}, 85 \%\right.$ yield) as a yellow oil $\left(R_{\mathrm{f}}=\right.$ 0.48 , ethyl acetate/petroleum $=1: 5) .[\alpha]_{\mathrm{D}}^{27}+41.4\left(c 1.0, \mathrm{CHCl}_{3}\right) .{ }^{1} \mathrm{H} \mathrm{NMR}\left(400 \mathrm{MHz}, \mathrm{CDCl}_{3}\right) \delta 10.57(\mathrm{~s}, 1 \mathrm{H}), 9.10(\mathrm{~s}, 1 \mathrm{H}), 6.63$ $(\mathrm{s}, 1 \mathrm{H}), 6.52(\mathrm{~s}, 1 \mathrm{H}), 5.62(\mathrm{~s}, 1 \mathrm{H}), 4.69(\mathrm{~s}, 1 \mathrm{H}), 3.87(\mathrm{~s}, 3 \mathrm{H}), 2.97(\mathrm{dd}, J=16.4,2.0 \mathrm{~Hz}, 1 \mathrm{H}), 2.31(\mathrm{~s}, 3 \mathrm{H}), 1.97-1.86(\mathrm{~m}, 2 \mathrm{H})$, $1.46(\mathrm{~s}, 3 \mathrm{H}), 1.04(\mathrm{~d}, J=6.8 \mathrm{~Hz}, 3 \mathrm{H}), 0.87$ (d, $J=6.8 \mathrm{~Hz}, 3 \mathrm{H}) .{ }^{13} \mathrm{C} \mathrm{NMR}\left(101 \mathrm{MHz}, \mathrm{CDCl}_{3}\right) \delta 205.3(1 \mathrm{C}, \mathrm{CHO}), 192.3(1 \mathrm{C}$, CHO), 163.5 (1C, C), 150.7 (1C, C), 146.2 (1C, C), 141.9 (1C, C), 122.0 (1C, CH), $121.6(1 \mathrm{C}, \mathrm{CH}), 121.3(1 \mathrm{C}, \mathrm{C}), 110.6(1 \mathrm{C}$, $\mathrm{CH}), 58.0(1 \mathrm{C}, \mathrm{C}), 55.7(1 \mathrm{C}, \mathrm{CH}), 55.7\left(1 \mathrm{C}, \mathrm{CH}_{3}\right), 38.4\left(1 \mathrm{C}, \mathrm{CH}_{2}\right), 27.8(1 \mathrm{C}, \mathrm{CH}), 23.4\left(1 \mathrm{C}, \mathrm{CH}_{3}\right), 22.5\left(1 \mathrm{C}, \mathrm{CH}_{3}\right), 21.8(1 \mathrm{C}$, $\left.\mathrm{CH}_{3}\right), 21.3\left(1 \mathrm{C}, \mathrm{CH}_{3}\right)$. IR (KBr): $v_{\max }$ 2961, 2929, 2870, 1720, 1679, 1648, 1605, 1569, 1458, 1407, 1296, 1279, 1195, 1141, 1079, $740 \mathrm{~cm}^{-1}$ HRMS (ESI) Calcd for $\mathrm{C}_{19} \mathrm{H}_{25} \mathrm{O}_{3}\left([\mathrm{M}+\mathrm{H}]^{+}\right)$: 301.1798; Found: 301.1796.

\section{(C) Synthesis of (-)-Hamigeran B and (-)-4-Bromohamigeran B}

\section{Synthesis of compound 6.}

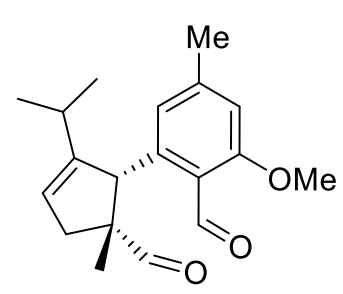

5

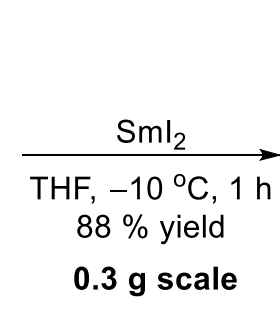

$0.3 \mathrm{~g}$ scale

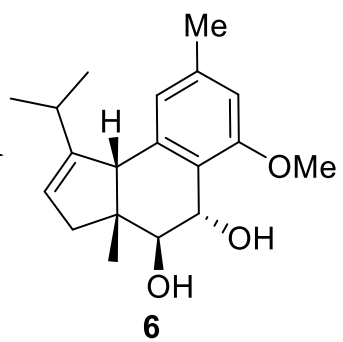

To a solution of $\mathrm{SmI}_{2}(80 \mathrm{~mL}, 8 \mathrm{mmol}, 0.1 \mathrm{M}$ in THF $)$ was added compound $\mathbf{5}(300 \mathrm{mg}, 1 \mathrm{mmol})$ at $-10^{\circ} \mathrm{C}$. The reaction mixture was stiired at the same temperature for $1 \mathrm{~h}, \mathrm{H}_{2} \mathrm{O}(20 \mathrm{~mL})$ was then added. The resulting mixture was extracted with EtOAc $(3 \times 10 \mathrm{~mL})$, and the combined extracts were washed with brine, dried over $\mathrm{Na}_{2} \mathrm{SO}_{4}$, and concentrated in vacuo. The residue was purified by chromatography on silica gel (ethyl acetate/petroleum $=1: 1)$ to afford 6 (266 $\mathrm{mg}, 88 \%$ yield) as a single diastereomer as a colorless oil $\left(R_{\mathrm{f}}=0.15\right.$, ethyl acetate/petroleum $\left.=1: 5\right) .[\alpha]_{\mathrm{D}}^{30}+130.2\left(c 1.0, \mathrm{CHCl}_{3}\right) .{ }^{1} \mathrm{H} \mathrm{NMR}\left(400 \mathrm{MHz}, \mathrm{CDCl}_{3}\right)$ $\delta 6.68(\mathrm{~s}, 1 \mathrm{H}), 6.60(\mathrm{~s}, 1 \mathrm{H}), 5.35(\mathrm{~s}, 1 \mathrm{H}), 4.78(\mathrm{~d}, J=8.0 \mathrm{~Hz}, 1 \mathrm{H}), 4.25(\mathrm{~s}, 1 \mathrm{H}), 3.88(\mathrm{~s}, 3 \mathrm{H}), 3.85(\mathrm{~s}, 1 \mathrm{H}), 3.55(\mathrm{~s}, 1 \mathrm{H}), 2.74(\mathrm{~d}$, $J=16.0 \mathrm{~Hz}, 1 \mathrm{H}), 2.61(\mathrm{~s}, 1 \mathrm{H}), 2.34(\mathrm{~s}, 3 \mathrm{H}), 2.24(\mathrm{~m}, 1 \mathrm{H}), 2.03(\mathrm{~m}, 1 \mathrm{H}), 1.12(\mathrm{~m}, 6 \mathrm{H}), 0.77(\mathrm{~d}, J=6.8 \mathrm{~Hz}, 3 \mathrm{H}) .{ }^{13} \mathrm{C} \mathrm{NMR}(101$ $\left.\mathrm{MHz}, \mathrm{CDCl}_{3}\right) \delta 158.1(1 \mathrm{C}, \mathrm{C}), 152.5$ (1C, C), 138.0 (1C, C), $136.9(1 \mathrm{C}, \mathrm{C}), 123.7(1 \mathrm{C}, \mathrm{CH}), 122.3(1 \mathrm{C}, \mathrm{C}), 119.4(1 \mathrm{C}, \mathrm{CH})$, $109.3(1 \mathrm{C}, \mathrm{CH}), 73.6(1 \mathrm{C}, \mathrm{CH}), 71.1(1 \mathrm{C}, \mathrm{CH}), 57.9(1 \mathrm{C}, \mathrm{CH}), 55.3\left(1 \mathrm{C}, \mathrm{CH}_{3}\right), 47.1\left(1 \mathrm{C}, \mathrm{CH}_{2}\right), 40.9(1 \mathrm{C}, \mathrm{C}), 27.0(1 \mathrm{C}, \mathrm{CH})$, $22.8\left(1 \mathrm{C}, \mathrm{CH}_{3}\right), 22.1\left(1 \mathrm{C}, \mathrm{CH}_{3}\right), 21.7\left(1 \mathrm{C}, \mathrm{CH}_{3}\right), 18.1\left(1 \mathrm{C}, \mathrm{CH}_{3}\right)$. IR $(\mathrm{KBr}): v_{\max } 3685,3458,3050,2958,2932,2869,2839,1726$, $1634,1613,1582,1461,1381,1333,1259,1144,1124,1088,1044,1010,969,829,800 \mathrm{~cm}^{-1} \mathrm{HRMS}_{(\mathrm{ESI})} \mathrm{Calcd} \mathrm{for} \mathrm{C}_{19} \mathrm{H}_{26} \mathrm{NaO}_{3}$ $\left([\mathrm{M}+\mathrm{Na}]^{+}\right)$: 325.1774; Found: 325.1778 .

\section{Synthesis of compound 25.}

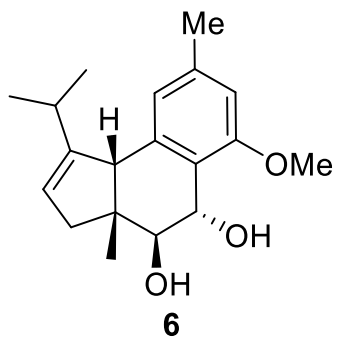

6

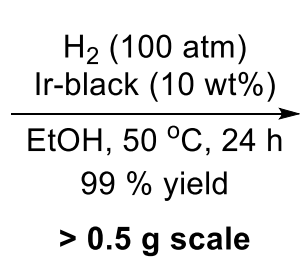

$0.5 \mathrm{~g}$ scale

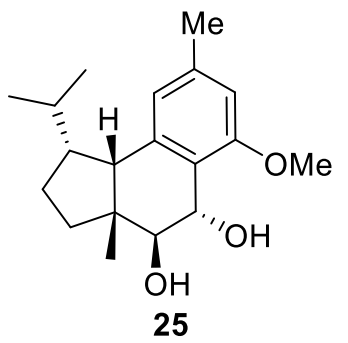


To a solution of compound $6(512 \mathrm{mg}, 1.68 \mathrm{mmol})$ in EtOH $(10 \mathrm{~mL})$ was added Ir-black $(51 \mathrm{mg}, 10 \mathrm{wt} \%)$. The reaction mixture was then charged with $100 \mathrm{~atm} \mathrm{H}_{2}$ and stirred at $50{ }^{\circ} \mathrm{C}$ for $24 \mathrm{~h}$. After filteration over a celite to remove the Ir-black, the solution was concentrated in vacuo. The residue was purified by chromatography on silica gel (ethyl acetate/petroleum $=1: 1$ ) to afford $25(512 \mathrm{mg}, 99 \%$ yield $)$ as a colorless oil $\left(R_{\mathrm{f}}=0.15\right.$, ethyl acetate/petroleum $\left.=1: 5\right) .[\alpha]_{\mathrm{D}}^{28}+16.2\left(c 1.0, \mathrm{CHCl}_{3}\right) .{ }^{1} \mathrm{H} \mathrm{NMR}$ $\left(400 \mathrm{MHz}, \mathrm{CDCl}_{3}\right) \delta 6.57(\mathrm{~s}, 1 \mathrm{H}), 6.54(\mathrm{~s}, 1 \mathrm{H}), 4.74(\mathrm{~d}, J=8.2 \mathrm{~Hz}, 1 \mathrm{H}), 4.27(\mathrm{~s}, 1 \mathrm{H}), 3.87(\mathrm{~s}, 3 \mathrm{H}), 3.83(\mathrm{~d}, J=8.4 \mathrm{~Hz}, 1 \mathrm{H}), 2.95$ $(\mathrm{d}, J=11.8 \mathrm{~Hz}, 1 \mathrm{H}), 2.56(\mathrm{~s}, 1 \mathrm{H}), 2.52-2.41(\mathrm{~m}, 1 \mathrm{H}), 2.32(\mathrm{~s}, 3 \mathrm{H}), 2.23-2.15(\mathrm{~m}, 1 \mathrm{H}), 1.84-1.75(\mathrm{~m}, 1 \mathrm{H}), 1.75-1.66(\mathrm{~m}, 2 \mathrm{H})$, $1.33-1.26(\mathrm{~m}, 1 \mathrm{H}), 0.99(\mathrm{~s}, 3 \mathrm{H}), 0.75(\mathrm{~d}, J=6.8 \mathrm{~Hz}, 3 \mathrm{H}), 0.49(\mathrm{~d}, J=6.8 \mathrm{~Hz}, 3 \mathrm{H}) .{ }^{13} \mathrm{C} \mathrm{NMR}\left(101 \mathrm{MHz} \mathrm{CDCl}_{3}\right) \delta 157.5(1 \mathrm{C}$, Ar-C), 137.7 (1C, Ar-C), 137.5 (1C, Ar-C), 124.4 (1C, Ar-CH), 122.7 (1C, Ar-C), 108.7 (1C, Ar-CH), 73.1 (1C, CH), 71.2 (1C, $\mathrm{CH}), 55.3\left(1 \mathrm{C}, \mathrm{CH}_{3}\right), 54.0(1 \mathrm{C}, \mathrm{CH}), 47.8(1 \mathrm{C}, \mathrm{CH}), 45.3(1 \mathrm{C}, \mathrm{C}), 35.8\left(1 \mathrm{C}, \mathrm{CH}_{2}\right), 28.2(1 \mathrm{C}, \mathrm{CH}), 24.0(1 \mathrm{C}, \mathrm{CH}), 23.7(1 \mathrm{C}$, $\left.\mathrm{CH}_{3}\right), 21.6\left(1 \mathrm{C}, \mathrm{CH}_{3}\right), 19.7\left(1 \mathrm{C}, \mathrm{CH}_{3}\right), 18.1$ (1C, $\left.\mathrm{CH}_{3}\right)$. IR (KBr): v $\max 3605,3469,2951,2874,1649,1614,1581,1461,1384$, 1334, 1263, 1091, 1064, 1040, 1008, 763, $749 \mathrm{~cm}^{-1}$ HRMS (ESI) Calcd for $\mathrm{C}_{19} \mathrm{H}_{28} \mathrm{NaO}_{3}\left([\mathrm{M}+\mathrm{Na}]^{+}\right)$: 327.1931 ; Found: 327.1936.

The cis-oriented isopropyl group and aryl group on the five-membered ring and the trans-orientated hydroxyl groups on the six-membered ring were confirmed by nuclear Overhauser enhancement spectroscopy (see below).

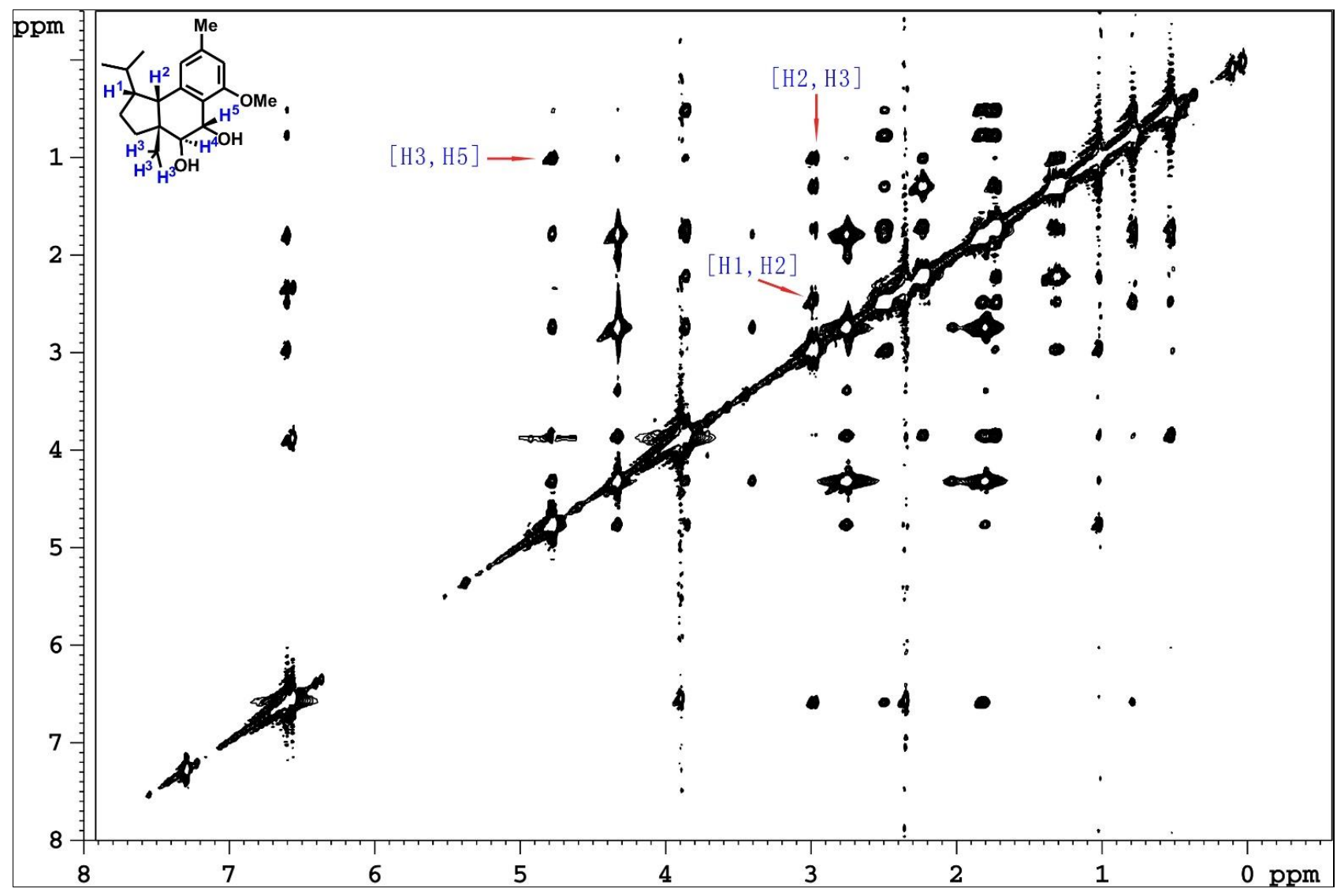

\section{Synthesis of compound 26.}




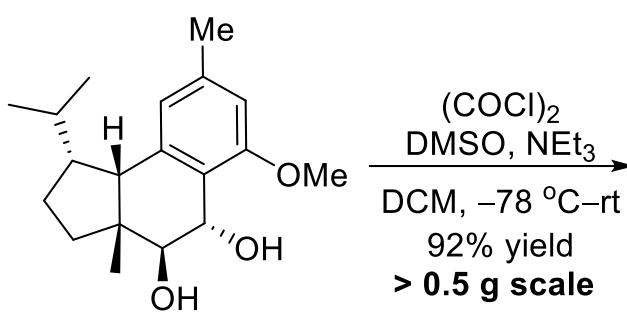

25

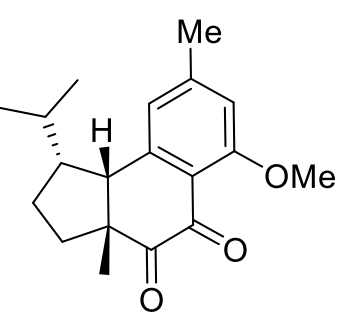

26

To a stirred solution of DMSO $(1.3 \mathrm{~g}, 16.8 \mathrm{mmol})$ in DCM $(5 \mathrm{~mL})$ was added $(\mathrm{COCl}){ }_{2}(1.1 \mathrm{~g}, 8.4 \mathrm{mmol})$ at $-78{ }^{\circ} \mathrm{C}$. The

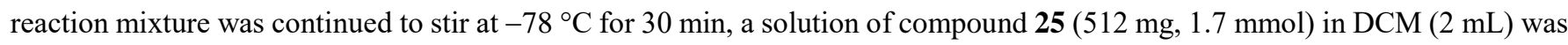
added dropwise over $5 \mathrm{~min}$. The resulting mixture was continued to stir at $-78{ }^{\circ} \mathrm{C}$ for $2 \mathrm{~h}, \mathrm{Et}_{3} \mathrm{~N}(3.40 \mathrm{~g}, 33.7 \mathrm{mmol}) \mathrm{was}$ then added dropwise to the solution. The reaction mixture was continuted to stir at $-78{ }^{\circ} \mathrm{C}$ f or $1 \mathrm{~h}$ and then allowed to warm to room temperature over $4 \mathrm{~h}$. Saturated aqueous $\mathrm{NH}_{4} \mathrm{Cl}$ solution $(15 \mathrm{~mL})$ was added, and the mixture was extracted with EtOAc $(3 \times 30$ $\mathrm{mL}$ ). The combined extracts were washed with brine, dried over $\mathrm{Na}_{2} \mathrm{SO}_{4}$, and concentrated in vacuo. The residue was purified by chromatography on silica gel (ethyl acetate/petroleum $=1: 3)$ to afford $\mathbf{2 6}(475 \mathrm{mg}, 94 \%)$ as a yellow oil $\left(R_{\mathrm{f}}=0.24\right.$, ethyl acetate/petroleum $=1: 5) .[\alpha]_{\mathrm{D}}^{30}-163.2\left(c 0.125, \mathrm{CH}_{2} \mathrm{Cl}_{2}\right) .{ }^{1} \mathrm{H} \mathrm{NMR}\left(400 \mathrm{MHz}, \mathrm{CDCl}_{3}\right) \delta 6.77(\mathrm{~s}, 1 \mathrm{H}), 6.70(\mathrm{~s}, 1 \mathrm{H}), 3.93(\mathrm{~s}, 3 \mathrm{H})$, $3.34(\mathrm{~d}, J=9.6 \mathrm{~Hz}, 1 \mathrm{H}), 2.52-2.45(\mathrm{~m}, 1 \mathrm{H}), 2.41(\mathrm{~s}, 3 \mathrm{H}), 2.28-2.18(\mathrm{~m}, 1 \mathrm{H}), 1.84-1.72(\mathrm{~m}, 1 \mathrm{H}), 1.58-1.46(\mathrm{~m}, 2 \mathrm{H}), 1.27(\mathrm{~s}$, $3 \mathrm{H}), 1.23-1.14(\mathrm{~m}, 1 \mathrm{H}), 0.57(\mathrm{~d}, J=6.6 \mathrm{~Hz}, 3 \mathrm{H}), 0.43(\mathrm{~d}, J=6.4 \mathrm{~Hz}, 3 \mathrm{H}) .{ }^{13} \mathrm{C} \mathrm{NMR}\left(101 \mathrm{MHz}, \mathrm{CDCl}_{3}\right) \delta 201.7(1 \mathrm{C}, \mathrm{C}=\mathrm{O})$, 180.8 (1C, C=O), 161.4 (1C, Ar-C), 147.2 (1C, Ar-C), 145.9 (1C, Ar-C), 124.4 (1C, Ar-CH), 120.5 (1C, Ar-C), 110.9 (1C, Ar$\mathrm{CH}), 56.0(1 \mathrm{C}, \mathrm{C}), 56.0(1 \mathrm{C}, \mathrm{CH}), 55.1\left(1 \mathrm{C}, \mathrm{CH}_{3}\right), 51.8(1 \mathrm{C}, \mathrm{CH}), 35.4\left(1 \mathrm{C}, \mathrm{CH}_{2}\right), 28.5(1 \mathrm{C}, \mathrm{CH}), 28.0\left(1 \mathrm{C}, \mathrm{CH}_{2}\right), 24.1(1 \mathrm{C}$, $\left.\mathrm{CH}_{3}\right), 23.1\left(1 \mathrm{C}, \mathrm{CH}_{3}\right), 22.4\left(1 \mathrm{C}, \mathrm{CH}_{3}\right), 20.3\left(1 \mathrm{C}, \mathrm{CH}_{3}\right)$. IR (KBr): v $\max 2953,2871,1677,1606,1459,1326,1264,1240,1103$, 1012, 839, $745 \mathrm{~cm}^{-1}$ HRMS (ESI) Calcd for $\mathrm{C}_{19} \mathrm{H}_{24} \mathrm{NaO}_{3}\left([\mathrm{M}+\mathrm{Na}]^{+}\right)$: 323.1618; Found: 323.1618 .

\section{Synthesis of compound 27.}
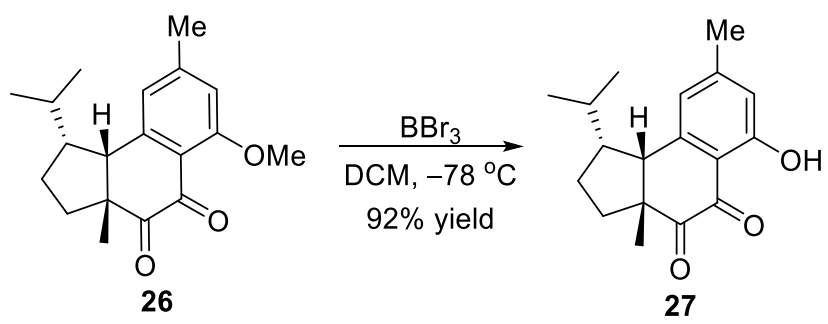

A solution of $\mathrm{BBr}_{3}\left(0.8 \mathrm{~mL}, 0.8 \mathrm{mmol}, 1.0 \mathrm{M}\right.$ in $\mathrm{CH}_{2} \mathrm{Cl}_{2}$ ) was slowly added to a solution of compound 26 (25 mg, 0.08 $\mathrm{mmol})$ in DCM $(2 \mathrm{~mL})$ at $-78{ }^{\circ} \mathrm{C}$. After the addition, the resulting mixture was allowed to warm to room temperature naturally and stirred at room temperature for $10 \mathrm{~h}$. Aqueous $\mathrm{NaOH}$ solution $(5 \mathrm{~mL}, 1 \mathrm{~N})$ was then added, and the mixture was continued to stir for $2 \mathrm{~h}$. The mixture was adjusted to neutral, and extracted with EtOAc $(3 \times 5 \mathrm{~mL})$. The combined extracts were dried over $\mathrm{Na}_{2} \mathrm{SO}_{4}$, and concentrated in vacuo. The residue was purified by chromatography on silica gel (acetone/hexanes $\left.=1: 20\right)$, to afford $27(21.1 \mathrm{mg}, 92 \%)$ as a light yellow solid $\left(R_{\mathrm{f}}=0.64\right.$, ethyl acetate/petroleum $\left.=1: 5\right)$. Mp 95-96 ${ }^{\circ} \mathrm{C},[\alpha]_{\mathrm{D}}^{27}-203.3(c 0.175$, $\left.\mathrm{CHCl}_{3}\right) .{ }^{1} \mathrm{H} \mathrm{NMR}\left(400 \mathrm{MHz}, \mathrm{CDCl}_{3}\right) \delta 11.92(\mathrm{~s}, 1 \mathrm{H}), 6.73(\mathrm{~s}, 1 \mathrm{H}), 6.69(\mathrm{~s}, 1 \mathrm{H}), 3.40(\mathrm{~d}, J=9.2 \mathrm{~Hz}, 1 \mathrm{H}), 2.62(\mathrm{~m}, 1 \mathrm{H}), 2.38(\mathrm{~s}$, $3 \mathrm{H}), 2.26(\mathrm{~m}, 1 \mathrm{H}), 1.80(\mathrm{~m}, 1 \mathrm{H}), 1.68(\mathrm{~m}, 1 \mathrm{H}), 1.60-1.49(\mathrm{~m}, 1 \mathrm{H}), 1.30(\mathrm{~s}, 3 \mathrm{H}), 1.21(\mathrm{~m}, 1 \mathrm{H}), 0.54(\mathrm{~d}, J=6.4 \mathrm{~Hz}, 3 \mathrm{H}), 0.42(\mathrm{~d}$, $J=6.4 \mathrm{~Hz}, 3 \mathrm{H}) .{ }^{13} \mathrm{C} \mathrm{NMR}\left(101 \mathrm{MHz}, \mathrm{CDCl}_{3}\right) \delta 200.1(1 \mathrm{C}, \mathrm{C}=\mathrm{O}), 184.4(1 \mathrm{C}, \mathrm{C}=\mathrm{O}), 164.7(1 \mathrm{C}, \mathrm{Ar}-\mathrm{C}), 150.8(1 \mathrm{C}, \mathrm{Ar}-\mathrm{C}), 144.2$ (1C, Ar-C), 123.4 (1C, Ar-CH), 116.7 (1C, Ar-C), 116.2 (1C, Ar-CH), 56.9 (1C, C), 56.5 (1C, CH), $51.4(1 \mathrm{C}, \mathrm{CH}), 33.8(1 \mathrm{C}$, $\left.\mathrm{CH}_{2}\right), 28.1(1 \mathrm{C}, \mathrm{CH}), 26.9\left(1 \mathrm{C}, \mathrm{CH}_{2}\right), 24.4\left(1 \mathrm{C}, \mathrm{CH}_{3}\right), 23.2\left(1 \mathrm{C}, \mathrm{CH}_{3}\right), 22.5\left(1 \mathrm{C}, \mathrm{CH}_{3}\right), 19.8\left(1 \mathrm{C}, \mathrm{CH}_{3}\right) . \mathrm{IR}(\mathrm{KBr}): v_{\max } 2961$, 1727, 1636, 1567, 1459, 1369, 1339, 1197, 1097, 1037, 937, 851, $741 \mathrm{~cm}^{-1}$ HRMS (ESI) Calcd for $\mathrm{C}_{18} \mathrm{H}_{22} \mathrm{NaO}_{3}\left([\mathrm{M}+\mathrm{Na}]^{+}\right)$: 309.1461; Found: 309.1462 . 


\section{Synthesis of (-)-hamigeran B. ${ }^{9}$}

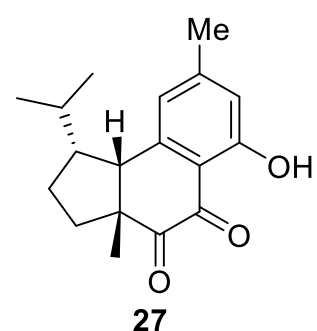

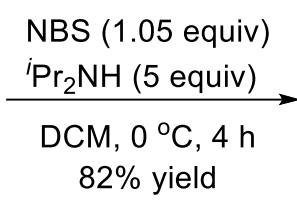

$82 \%$ yield

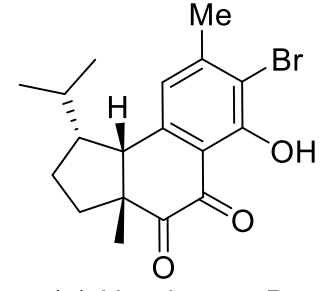

(-)-Hamigeran B

To a stirring solution of compound $27(10 \mathrm{mg}, 0.035 \mathrm{mmol})$ and ${ }^{i} \mathrm{Pr}_{2} \mathrm{NH}(18 \mathrm{mg}, 0.18 \mathrm{mmol})$ in DCM (4 mL) was added a solution of NBS $(6.5 \mathrm{mg}, 0.037 \mathrm{mmol})$ in DCM $(4 \mathrm{~mL})$ dropwise via a syringe pump over $4 \mathrm{~h}$ at $0{ }^{\circ} \mathrm{C}$. The mixture was then allowed to stir at room temperature for $3 \mathrm{~h}$. After concentrated the solution in vacuo, the residue was purified by flash chromatography on silica gel (acetone/hexane $=1: 20$ with small amount of $\mathrm{MeOH}$ ) to afford (-)-Hamigeran $\mathrm{B}(10 \mathrm{mg}, 82 \%$ yield $)$ as a yellow solid $\left(R_{\mathrm{f}}=0.48\right.$, ethyl acetate/petroleum $\left.=1: 5\right)$. Mp. $154-156{ }^{\circ} \mathrm{C}$ (lit., $\left.163-165{ }^{\circ} \mathrm{C}\right)^{11},[\alpha]_{\mathrm{D}}^{26}-167.3(c 0.11$, $\left.\mathrm{CHCl}_{3}\right)$ [natural, $\left.[\alpha]_{\mathrm{D}}^{25}-151.1\left(c 0.15, \mathrm{CH}_{2} \mathrm{Cl}_{2}\right)^{[8]}\right] .{ }^{1} \mathrm{H} \mathrm{NMR}\left(400 \mathrm{MHz}, \mathrm{CDCl}_{3}\right) \delta 12.65(\mathrm{~s}, 1 \mathrm{H}), 6.82(\mathrm{~s}, 1 \mathrm{H}), 3.39(\mathrm{~d}, J=9.2 \mathrm{~Hz}$, $1 \mathrm{H}), 2.69-2.57(\mathrm{~m}, 1 \mathrm{H}), 2.51(\mathrm{~s}, 3 \mathrm{H}), 2.38-2.25(\mathrm{~m}, 1 \mathrm{H}), 1.87-1.75(\mathrm{~m}, 1 \mathrm{H}), 1.75-1.63(\mathrm{~m}, 1 \mathrm{H}), 1.61-1.50(\mathrm{~m}, 1 \mathrm{H}), 1.29(\mathrm{~s}$, $3 \mathrm{H}), 1.26-1.10(\mathrm{~m}, 1 \mathrm{H}), 0.53(\mathrm{~d}, J=6.6 \mathrm{~Hz}, 3 \mathrm{H}), 0.45(\mathrm{~d}, J=6.6 \mathrm{~Hz}, 3 \mathrm{H}) .{ }^{13} \mathrm{C}$ NMR $(101 \mathrm{MHz}, \mathrm{CDCl} 3) \delta 199.0(1 \mathrm{C}, \mathrm{C}=\mathrm{O})$, $184.4(1 \mathrm{C}, \mathrm{C}=\mathrm{O}), 160.8$ (1C, Ar-C), 150.2 (1C, Ar-C), 142.7 (1C, Ar-C), 124.3 (1C, Ar-CH), 117.3 (1C, Ar-C), 111.6 (1C, ArC), $56.9(1 \mathrm{C}, \mathrm{C}), 56.2(1 \mathrm{C}, \mathrm{CH}), 51.3(1 \mathrm{C}, \mathrm{CH}), 33.8\left(1 \mathrm{C}, \mathrm{CH}_{2}\right), 28.1(1 \mathrm{C}, \mathrm{CH}), 26.8\left(1 \mathrm{C}, \mathrm{CH}_{2}\right), 24.4\left(1 \mathrm{C}, \mathrm{CH}_{3}\right), 24.3\left(1 \mathrm{C}, \mathrm{CH}_{3}\right)$, $23.3\left(1 \mathrm{C}, \mathrm{CH}_{3}\right), 19.7\left(1 \mathrm{C}, \mathrm{CH}_{3}\right)$. IR (KBr): $v_{\max } 3420,2960,2924,2904,2869,1723,1628,1451,1385,1330,1297,1170,1097$, 1030, $779 \mathrm{~cm}^{-1} \mathrm{HRMS}(\mathrm{ESI})$ Calcd for $\mathrm{C}_{18} \mathrm{H}_{21} \mathrm{BrNaO}_{3}\left([\mathrm{M}+\mathrm{Na}]^{+}\right)$: 387.0566; Found: 387.0569 .

\section{Synthesis of (-)-4-Bromohamigeran B. ${ }^{9}$}

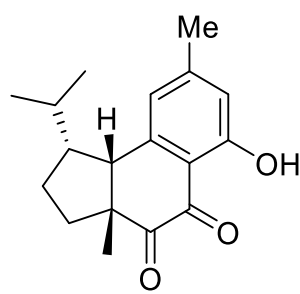

27
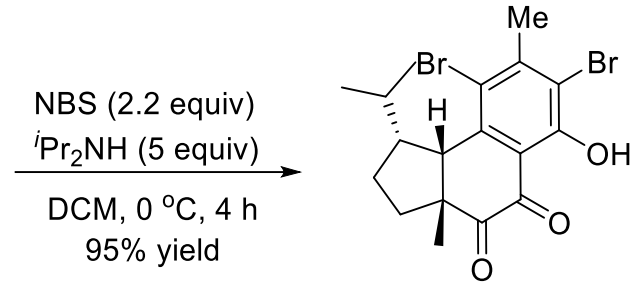

$(-)-4-B r o m o h a m i g e r a n ~ B$

To a stirring solution of compound $27(10 \mathrm{mg}, 0.035 \mathrm{mmol})$ and ${ }^{i} \operatorname{Pr}_{2} \mathrm{NH}(18 \mathrm{mg}, 0.18 \mathrm{mmol})$ in DCM (4 mL) was added a solution of NBS $(13.7 \mathrm{mg}, 0.077 \mathrm{mmol})$ in DCM $(4 \mathrm{~mL})$ dropwise via a syringe pump over $4 \mathrm{~h}$ at $0{ }^{\circ} \mathrm{C}$. The mixture was then allowed to stir at room temperature for $3 \mathrm{~h}$. After concentrated the solution in vacuo, the residue was purified by flash chromatography on silica gel (acetone/hexane $=1: 20$, with small amount of $\mathrm{MeOH}$ ) to afford (-)-4-Bromohamigeran $\mathrm{B}(15$ $\mathrm{mg}, 95 \%$ yield $)$ as a yellow solid $\left(R_{\mathrm{f}}=0.36\right.$, ethyl acetate/petroleum $\left.=1: 5\right) . \mathrm{Mp} 147-150{ }^{\circ} \mathrm{C}\left(\text { lit., } 144-148^{\circ} \mathrm{C}\right)^{11},[\alpha]_{\mathrm{D}}^{26}-80.0(c$ $\left.0.10, \mathrm{CHCl}_{3}\right)$ [natural, $\left.[\alpha]_{\mathrm{D}}^{25}-81.2\left(c 0.37, \mathrm{CH}_{2} \mathrm{Cl}_{2}\right)^{[8]}\right] .{ }^{1} \mathrm{H} \mathrm{NMR}\left(400 \mathrm{MHz}, \mathrm{CDCl}_{3}\right) \delta 12.94(\mathrm{~s}, 1 \mathrm{H}), 3.71(\mathrm{~d}, J=10.2 \mathrm{~Hz}, 1 \mathrm{H})$, $2.79(\mathrm{~s}, 3 \mathrm{H}), 2.77-2.73(\mathrm{~m}, 1 \mathrm{H}), 2.67(\mathrm{~m}, 1 \mathrm{H}), 1.91-1.81(\mathrm{~m}, 1 \mathrm{H}), 1.73-1.63(\mathrm{~m}, 1 \mathrm{H}), 1.54-1.50(\mathrm{~m}, 1 \mathrm{H}), 1.28(\mathrm{~s}, 3 \mathrm{H}), 1.23-$ $1.18(\mathrm{~m}, 1 \mathrm{H}), 0.52(\mathrm{~d}, J=6.6 \mathrm{~Hz}, 3 \mathrm{H}), 0.46(\mathrm{~d}, J=6.6 \mathrm{~Hz}, 3 \mathrm{H}) .{ }^{13} \mathrm{C} \mathrm{NMR}\left(101 \mathrm{MHz}, \mathrm{CDCl}_{3}\right) \delta 198.0(1 \mathrm{C}, \mathrm{C}=\mathrm{O}), 185.0(1 \mathrm{C}$, $\mathrm{C}=\mathrm{O}), 160.1$ (1C, Ar-C), 149.8 (1C, Ar-C), 142.1 (1C, Ar-C), 119.3 (1C, Ar-C), 117.6 (1C, Ar-C), 113.3 (1C, Ar-C), 57.8 (1C, $\mathrm{CH}), 57.7(1 \mathrm{C}, \mathrm{C}), 47.4(1 \mathrm{C}, \mathrm{CH}), 33.7\left(1 \mathrm{C}, \mathrm{CH}_{2}\right), 28.5(1 \mathrm{C}, \mathrm{CH}), 26.6\left(1 \mathrm{C}, \mathrm{CH}_{3}\right), 25.3\left(1 \mathrm{C}, \mathrm{CH}_{2}\right), 23.5\left(1 \mathrm{C}, \mathrm{CH}_{3}\right), 22.9(1 \mathrm{C}$, $\left.\mathrm{CH}_{3}\right), 19.0\left(1 \mathrm{C}, \mathrm{CH}_{3}\right) . \mathrm{IR}(\mathrm{KBr}): v_{\max } 3438,2956,2924,2869,1728,1713,1610,1561,1450,1388,1373,1186 \mathrm{~cm}^{-1} ; \mathrm{HRMS}^{-}$ (ESI) Calcd for $\mathrm{C}_{18} \mathrm{H}_{21} \mathrm{Br}_{2} \mathrm{O}_{3}\left([\mathrm{M}+\mathrm{H}]^{+}\right)$: 442.9852; Found: 442.9842 . 


\section{Synthesis of compounds $14 .^{10}$}

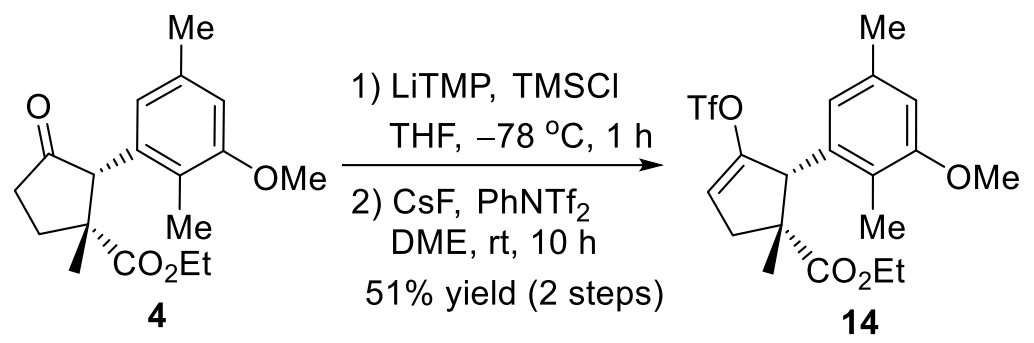

To a stirred solution of 2,2,6,6-tetramethylpiperidine (440 mg, $3.12 \mathrm{mmol})$ in dry THF $(5 \mathrm{~mL})$ at $0{ }^{\circ} \mathrm{C}$ was added dropwise a solution of $n$-BuLi $\left(1.30 \mathrm{~mL}, 3.12 \mathrm{mmol}, 2.4 \mathrm{M}\right.$ in hexane) over $5 \mathrm{~min}$. After the solution was stirred at $0{ }^{\circ} \mathrm{C}$ for $30 \mathrm{~min}$, a solution of $\mathrm{TMSCl}(0.4 \mathrm{~mL}, 3.12 \mathrm{mmol})$ in THF $(1 \mathrm{~mL})$ was added and the resulting solution was continued to stirr at $-78{ }^{\circ} \mathrm{C}$ for $10 \mathrm{~min}$. A solution of $4(200 \mathrm{mg}, 0.66 \mathrm{mmol})$ in THF $(5 \mathrm{~mL})$ was then added slowly. The resulting solution was stirred at $-78{ }^{\circ} \mathrm{C}$ for $30 \mathrm{~min}$ to result a yellow solution. Saturated aqueuous $\mathrm{Na}_{2} \mathrm{CO}_{3}$ solution $(10 \mathrm{~mL})$ was added to quench the reaction, and the reaction mixture was extracted with $\mathrm{Et}_{2} \mathrm{O}(3 \times 10 \mathrm{~mL})$. The organic phase was dried over anhydrous $\mathrm{Na}_{2} \mathrm{SO}_{4}$, and concentrated in vacuo. The residue was dried in vacuo and redissolved in freshly distilled DME (2 mL). To the solution was added dry $\mathrm{PhNTf}_{2}(555 \mathrm{mg}, 1.56 \mathrm{~mol})$ and dry CsF $(380 \mathrm{mg}, 1.25 \mathrm{mmol})$, the resulting mixture was stirred at room temperature for $10 \mathrm{~h}$. The reaction mixture was then diluted with $\mathrm{Et}_{2} \mathrm{O}(10 \mathrm{~mL})$ and $\mathrm{pH}=7$ phosphate buffer $(5 \mathrm{~mL})$. The organic layer was separated and the aqueous layer was extracted with $\mathrm{Et}_{2} \mathrm{O}(3 \times 10 \mathrm{~mL})$. The combined extracts were washed with brine, dried over $\mathrm{Na}_{2} \mathrm{SO}_{4}$, and concentrated in vacuo. The residue was purified by chromatography on silica gel (ethyl acetate/petroleum $\left.=1: 10\right)$ to afford $14(146 \mathrm{mg}, 51 \%$ yield $)$ as a colorless oil $\left(R_{\mathrm{f}}=0.69\right.$, ethyl acetate/petroleum $\left.=1: 5\right) .[\alpha]_{\mathrm{D}}^{27}+74.7\left(c 0.3, \mathrm{CHCl}_{3}\right) .{ }^{1} \mathrm{H}$ NMR $\left(400 \mathrm{MHz}, \mathrm{CDCl}_{3}\right) \delta 6.54(\mathrm{~s}, 1 \mathrm{H}), 6.43(\mathrm{~s}, 1 \mathrm{H}), 5.88-5.84(\mathrm{~m}, 1 \mathrm{H}), 4.12-4.08(\mathrm{~m}, 1 \mathrm{H}), 3.78(\mathrm{~s}, 3 \mathrm{H}), 3.69-3.61(\mathrm{~m}, 1 \mathrm{H})$, 3.61-3.53 (m, 1H), 3.49-3.45 (m, 1H), 2.27-2.22 (m, 4H), $2.19(\mathrm{~s}, 3 \mathrm{H}), 1.59(\mathrm{~s}, 3 \mathrm{H}), 0.79(\mathrm{t}, J=7.1 \mathrm{~Hz}, 3 \mathrm{H}) .{ }^{13} \mathrm{C} \mathrm{NMR}(101$ $\left.\mathrm{MHz}, \mathrm{CDCl}_{3}\right) \delta 173.5(1 \mathrm{C}, \mathrm{C}=\mathrm{O}), 157.5$ (1C, C), 148.3 (1C, C), 135.7 (1C, C), $135.3(1 \mathrm{C}, \mathrm{C}), 123.1(1 \mathrm{C}, \mathrm{C}), 119.2(1 \mathrm{C}, \mathrm{CH})$, $117.0(1 \mathrm{C}, \mathrm{CH}), 110.6(1 \mathrm{C}, \mathrm{CH}), 60.7\left(1 \mathrm{C}, \mathrm{CH}_{2}\right), 55.6(1 \mathrm{C}, \mathrm{C}), 55.6(1 \mathrm{C}, \mathrm{CH}), 52.6(1 \mathrm{C}, \mathrm{C}), 37.6\left(1 \mathrm{C}, \mathrm{CH}_{2}\right), 27.9\left(1 \mathrm{C}, \mathrm{CH}_{3}\right)$, $21.5\left(1 \mathrm{C}, \mathrm{CH}_{3}\right), 13.3\left(1 \mathrm{C}, \mathrm{CH}_{3}\right), 11.1\left(1 \mathrm{C}, \mathrm{CH}_{3}\right) . \mathrm{IR}(\mathrm{KBr}): v_{\max } 2961,2935,2873,1731,1463,1214,1142,766 \mathrm{~cm}^{-1} \mathrm{HRMS}$ (ESI) Calcd for $\mathrm{C}_{19} \mathrm{H}_{23} \mathrm{~F}_{3} \mathrm{NaO}_{6} \mathrm{~S}\left([\mathrm{M}+\mathrm{Na}]^{+}\right)$: 459.1060; Found: 459.1065 .

\section{Synthesis of compounds 17.}

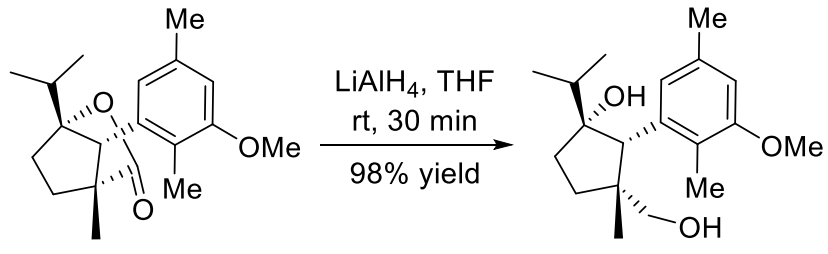

16

17

To a solution of compound $16(100 \mathrm{mg}, 0.33 \mathrm{mmol})$ in THF $(5 \mathrm{~mL})$ was added $\mathrm{LiAlH}_{4}(25 \mathrm{mg}, 0.66 \mathrm{mmol})$ at $0{ }^{\circ} \mathrm{C}$, and reaction mixture was stirred for $30 \mathrm{~min}$ at room temperature. EtOAc $(10 \mathrm{~mL})$ was then added to quench the reaction. After the reaction mixture was stirred for additional $1 \mathrm{~h}$, saturated aqueous potassium sodium tartrate solution $(10 \mathrm{~mL})$ was added. The mixture was continued to stirr for $30 \mathrm{~min}$, the organic layer was separated and the aqueous layer was extracted with EtOAc $(3 \times$ $10 \mathrm{~mL}$ ). The combined extracts were washed with brine, dried over $\mathrm{Na}_{2} \mathrm{SO}_{4}$, and concentrated in vacuo. The residue was purified by chromatography on silica gel (ethyl acetate/petroleum $=1: 2)$ to afford $\mathbf{1 7}\left(88 \mathrm{mg}, 88 \%\right.$ yield) as a white solid $\left(R_{\mathrm{f}}=0.3\right.$, ethyl acetate/petroleum = 1:3). Mp: $174-176{ }^{\circ} \mathrm{C},[\alpha]_{\mathrm{D}}^{27}+18.0\left(c 0.1, \mathrm{CHCl}_{3}\right) .{ }^{1} \mathrm{H} \mathrm{NMR}\left(400 \mathrm{MHz}, \mathrm{CDCl}_{3}\right) \delta 7.12(\mathrm{~s}, 1 \mathrm{H}), 6.61(\mathrm{~s}, 1 \mathrm{H})$, $3.81(\mathrm{~s}, 3 \mathrm{H}), 3.41(\mathrm{~d}, J=11.2 \mathrm{~Hz}, 1 \mathrm{H}), 3.25(\mathrm{~d}, J=11.2 \mathrm{~Hz}, 1 \mathrm{H}), 3.22(\mathrm{~s}, 1 \mathrm{H}), 2.34(\mathrm{~s}, 3 \mathrm{H}), 2.23-2.12(\mathrm{~m}, 4 \mathrm{H}), 2.02-1.92(\mathrm{~m}$, 
1H), $1.80-1.69(\mathrm{~m}, 1 \mathrm{H}), 1.65-1.49(\mathrm{~m}, 3 \mathrm{H}), 0.95(\mathrm{~s}, 3 \mathrm{H}), 0.86(\mathrm{~d}, J=6.8 \mathrm{~Hz}, 3 \mathrm{H}), 0.77(\mathrm{~d}, J=6.8 \mathrm{~Hz}, 3 \mathrm{H}) .{ }^{13} \mathrm{C} \mathrm{NMR}(101 \mathrm{MHz}$, $\left.\mathrm{CDCl}_{3}\right) \delta 157.7$ (1C, Ar-C), 137.2 (1C, Ar-C), 135.1 (1C, Ar-C), 123.6 (1C, Ar-C), 122.5 (1C, Ar-CH), 109.5 (1C, Ar-CH), 86.9 $(1 \mathrm{C}, \mathrm{C}), 68.1\left(1 \mathrm{C}, \mathrm{CH}_{2}\right), 55.4(1 \mathrm{C}, \mathrm{CH}), 48.4(1 \mathrm{C}, \mathrm{C}), 36.1(1 \mathrm{C}, \mathrm{CH}), 34.7\left(1 \mathrm{C}, \mathrm{CH}_{2}\right), 33.3\left(1 \mathrm{C}, \mathrm{CH}_{2}\right), 26.3\left(1 \mathrm{C}, \mathrm{CH}_{3}\right), 22.0(1 \mathrm{C}$, $\left.\mathrm{CH}_{3}\right), 18.7\left(1 \mathrm{C}, \mathrm{CH}_{3}\right), 17.7\left(1 \mathrm{C}, \mathrm{CH}_{3}\right), 12.2\left(1 \mathrm{C}, \mathrm{CH}_{3}\right) . \mathrm{IR}(\mathrm{KBr}): v_{\max } 3215,3118,2951,2934,2870,1463,1296,1141,1098$, 1043, 1007, 806, $770 \mathrm{~cm}^{-1} \mathrm{HRMS}$ (ESI) Calcd for $\mathrm{C}_{19} \mathrm{H}_{30} \mathrm{NaO}_{3}\left([\mathrm{M}+\mathrm{Na}]^{+}\right)$: 329.2087; Found: 329.2082.

\section{References:}

(1) Krafft, M. E.; Cran, J. W. Synlett 2005, 1263-1266.

(2) Gore, M. P.; Gould, S. J.; Weller, D. D. J. Org. Chem. 1992, 57, 2774-2783.

(3) Wolfe, J. P.; Singer, R. A.; Yang,B.-H.; Buchwald, S. L. J. Am. Chem. Soc. 1999, 121, 9550-9561.

(4) Yang, J.-Y.; Wang, Y.-X.; Wu, S.-X.; Chen, F.-X. Synlett 2009, 3365-3367.

(5) Bartoszewicz, A.; Kalek, M.; Nilsson, J.; Hiresova, R.; Stawinski, J. Synlett 2008, 37-40.

(6) Cossfo, F. P.; Aizpurua, J.; Palomo, C. Can. J. Chem. 1986, 64, 225-231.

(7) Nicolaou, K. C.; Gray, D. L. F.; Tae, J. J. Am. Chem. Soc. 2004, 126, 613-627

(8) Wellington, K. D.; Cambie, R. C.; Rutledge, P. S.; Bergquist, P. R. J. Nat. Prod. 2000, 63, 79-85.

(9) Trost, B. M.; Pissot-Soldermann, C.; Chen, I.; Schroeder, G. M. J. Am. Chem. Soc. 2004, 126, 4480-4481.

(10) Mi, Y.; Schreiber, J. V.; Corey, E. J. J. Am. Chem. Soc. 2002, 124, 11290-11291.

(11) Wellington, K. D.; Cambie, R. C.; Rutledge, P. S.; Bergquist, P. R. J. Nat. Prod. 2000, 63, 79-85 
(E) NMR Spectra of New Compounds

\section{Compound 9.}
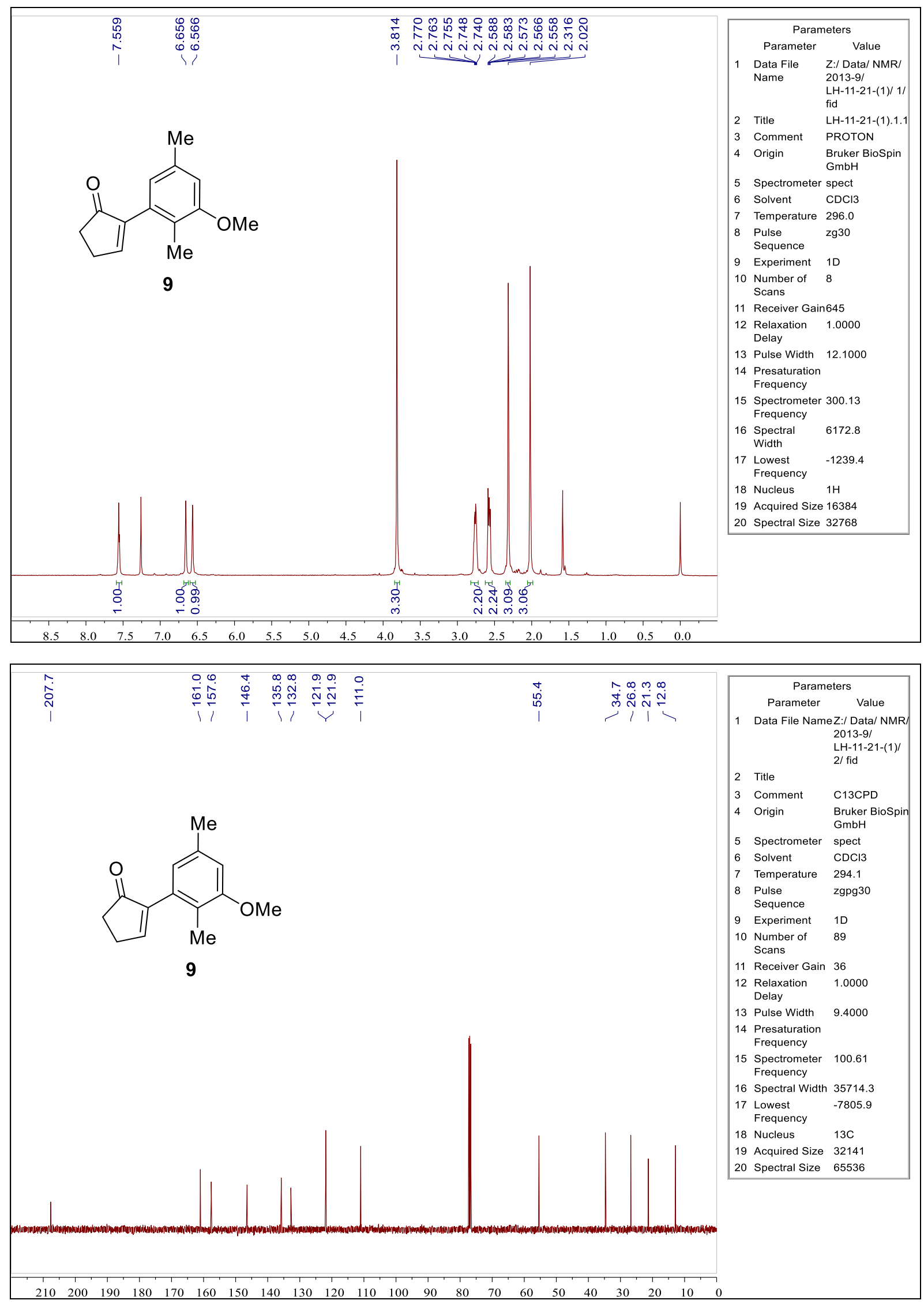
Compound 10.

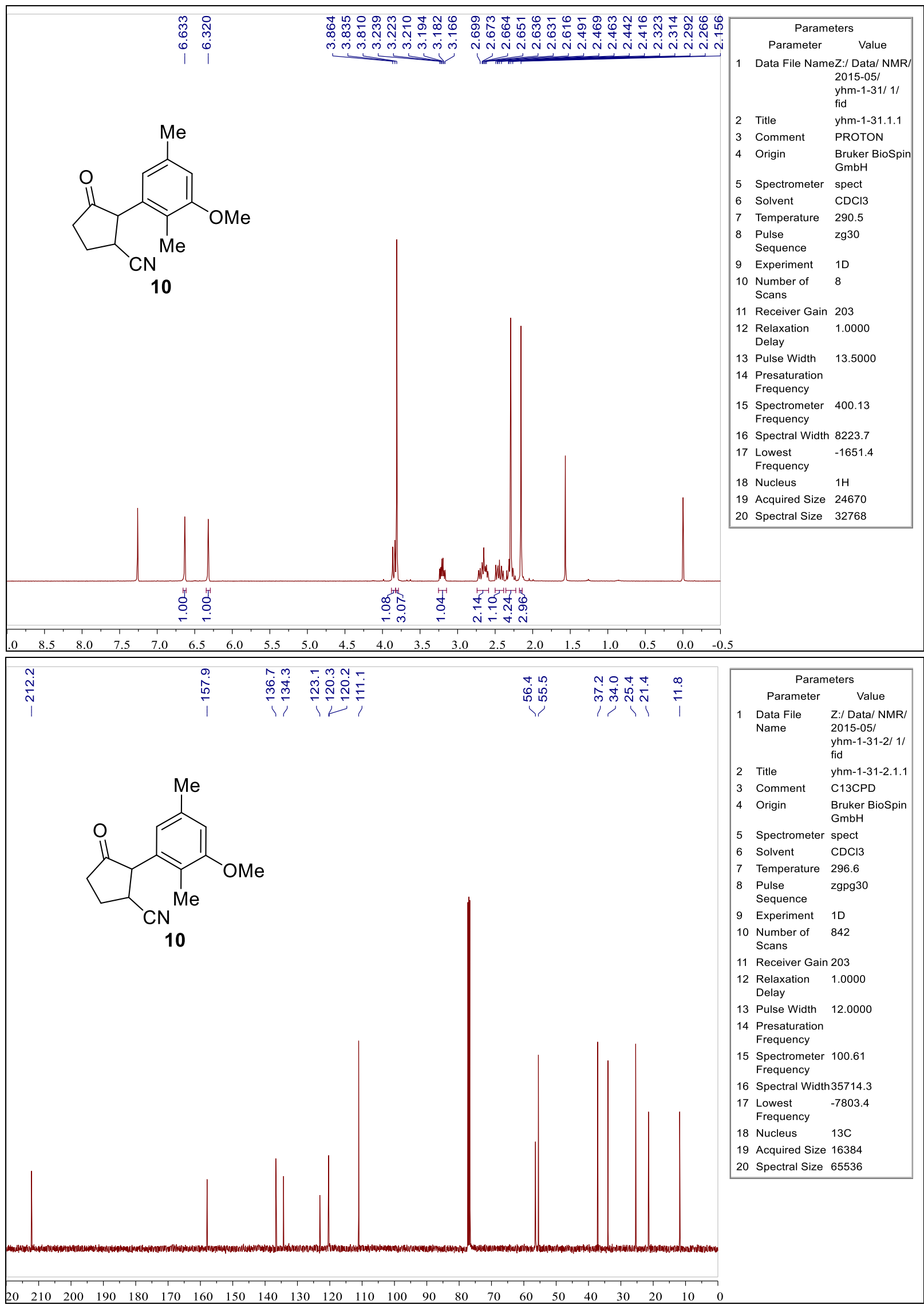




\section{Compound trans-2}
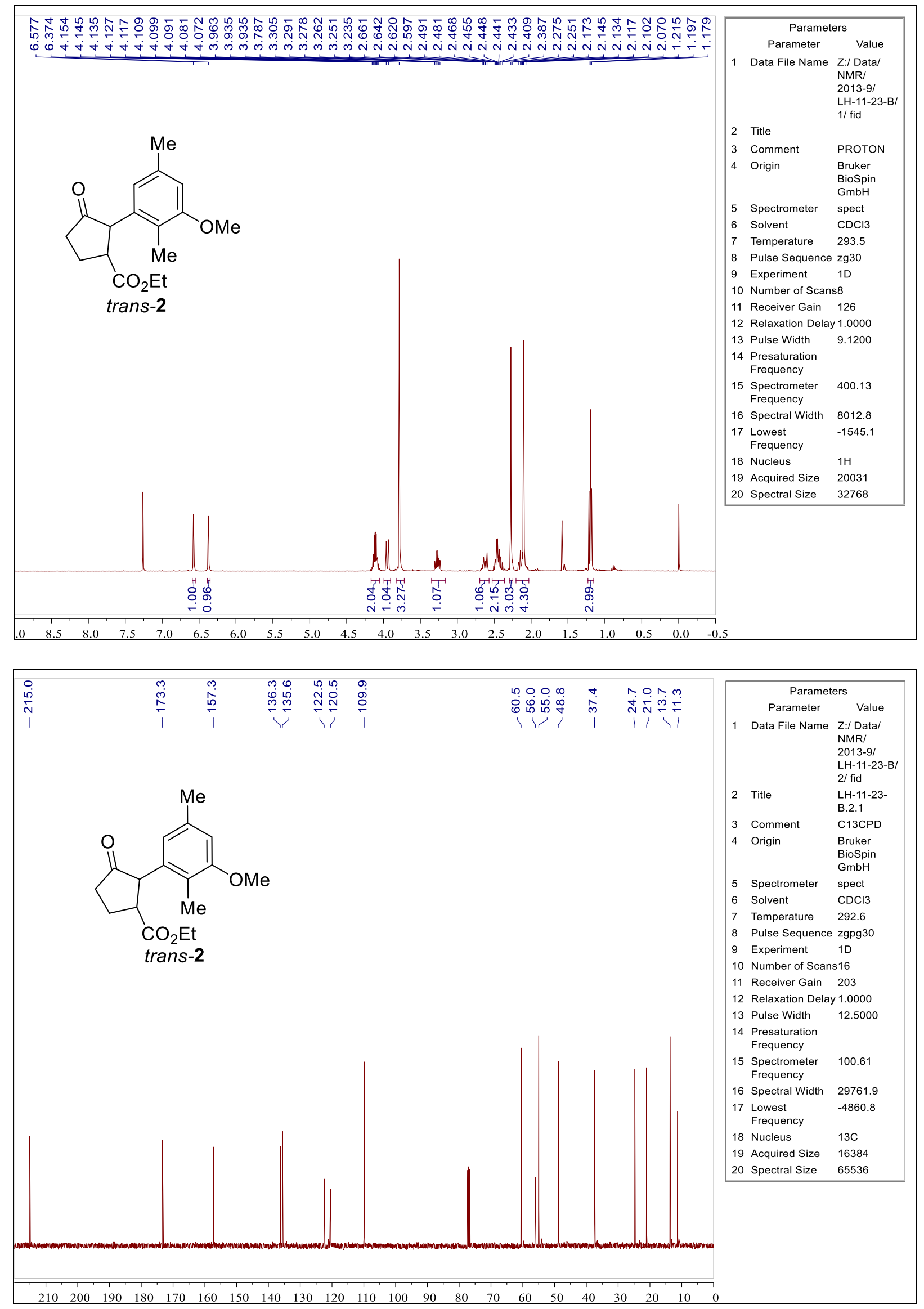


\section{Compound (+)-3.}

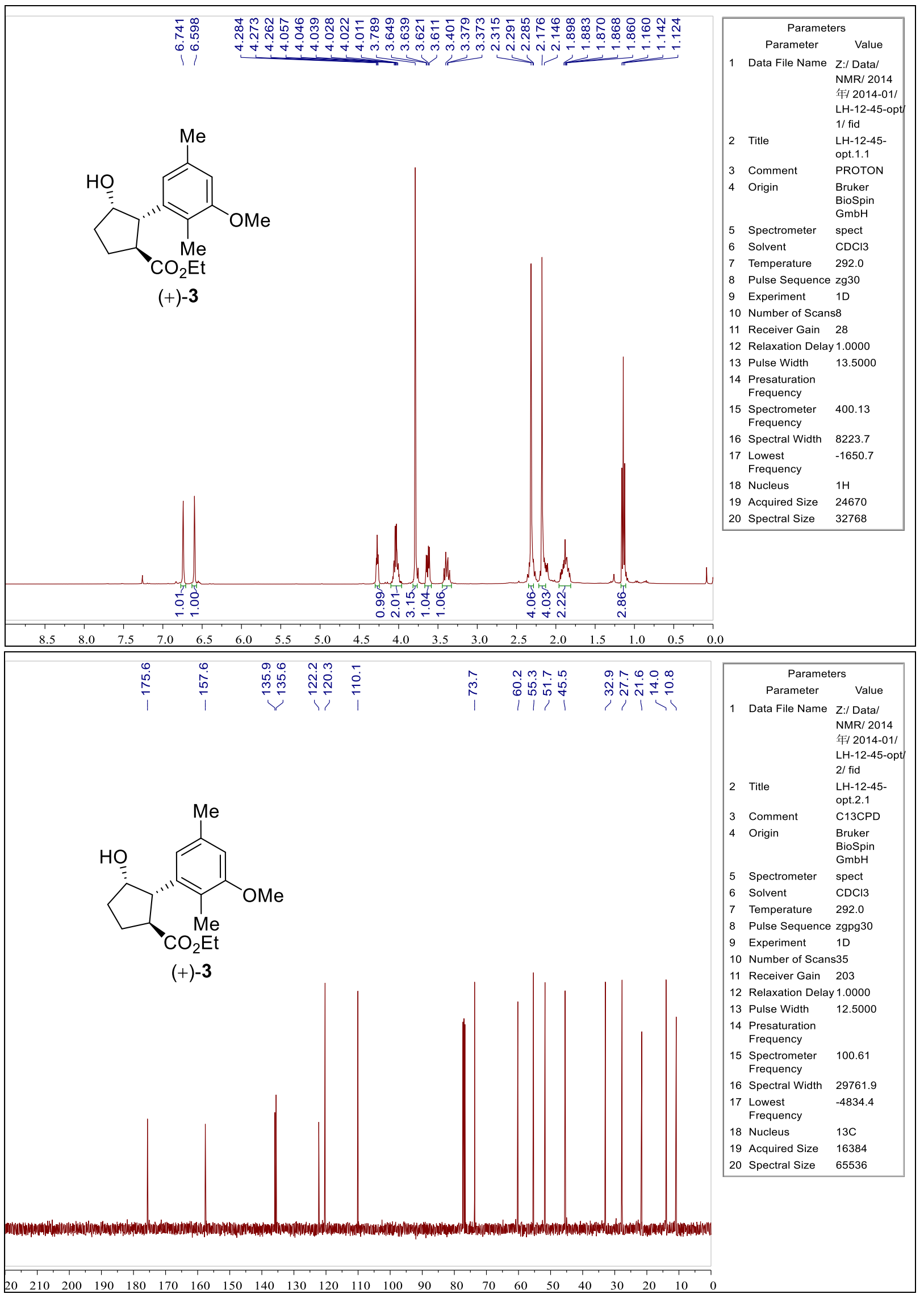


Compound 12.

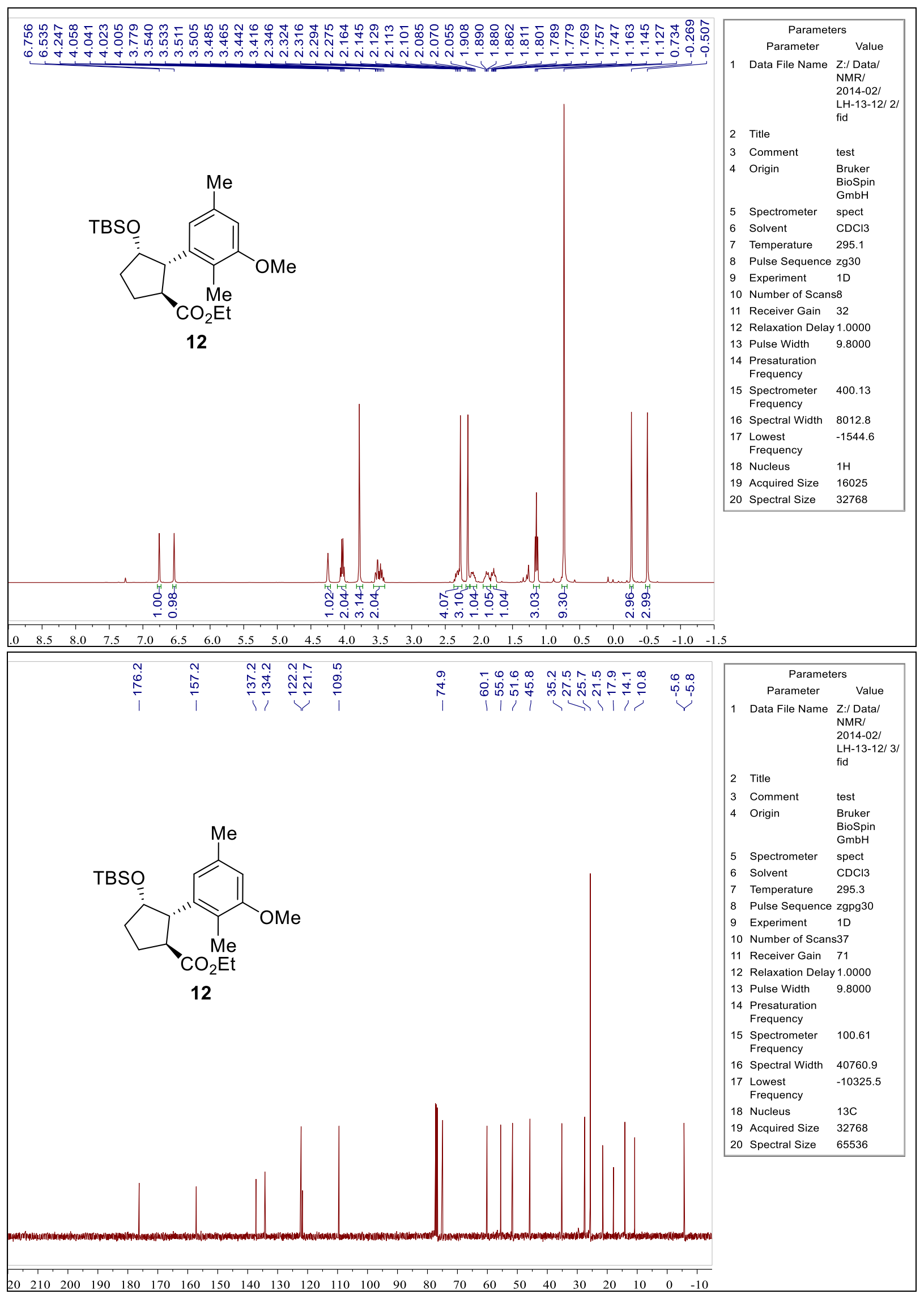


Compound 13.

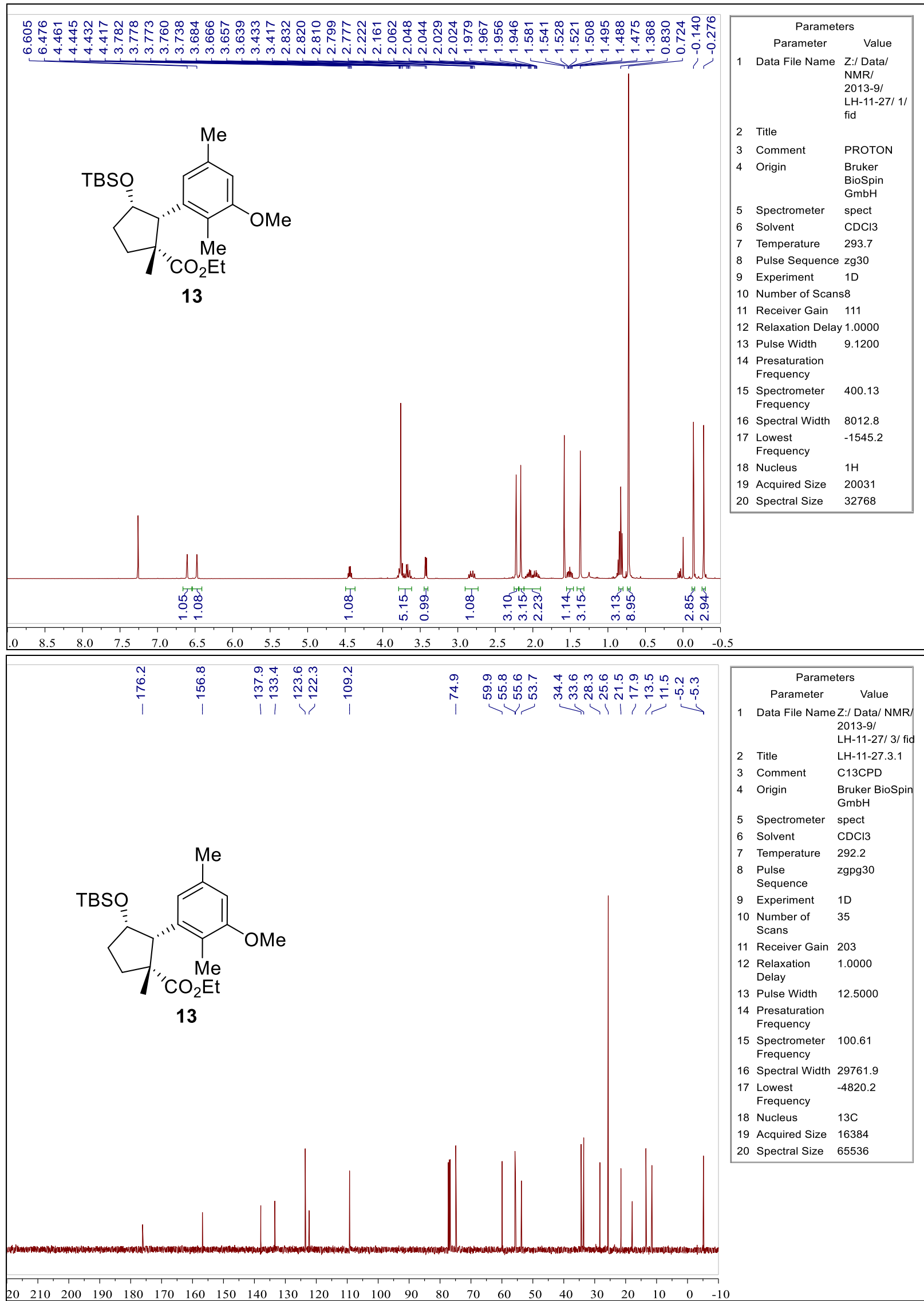


Compound 4.
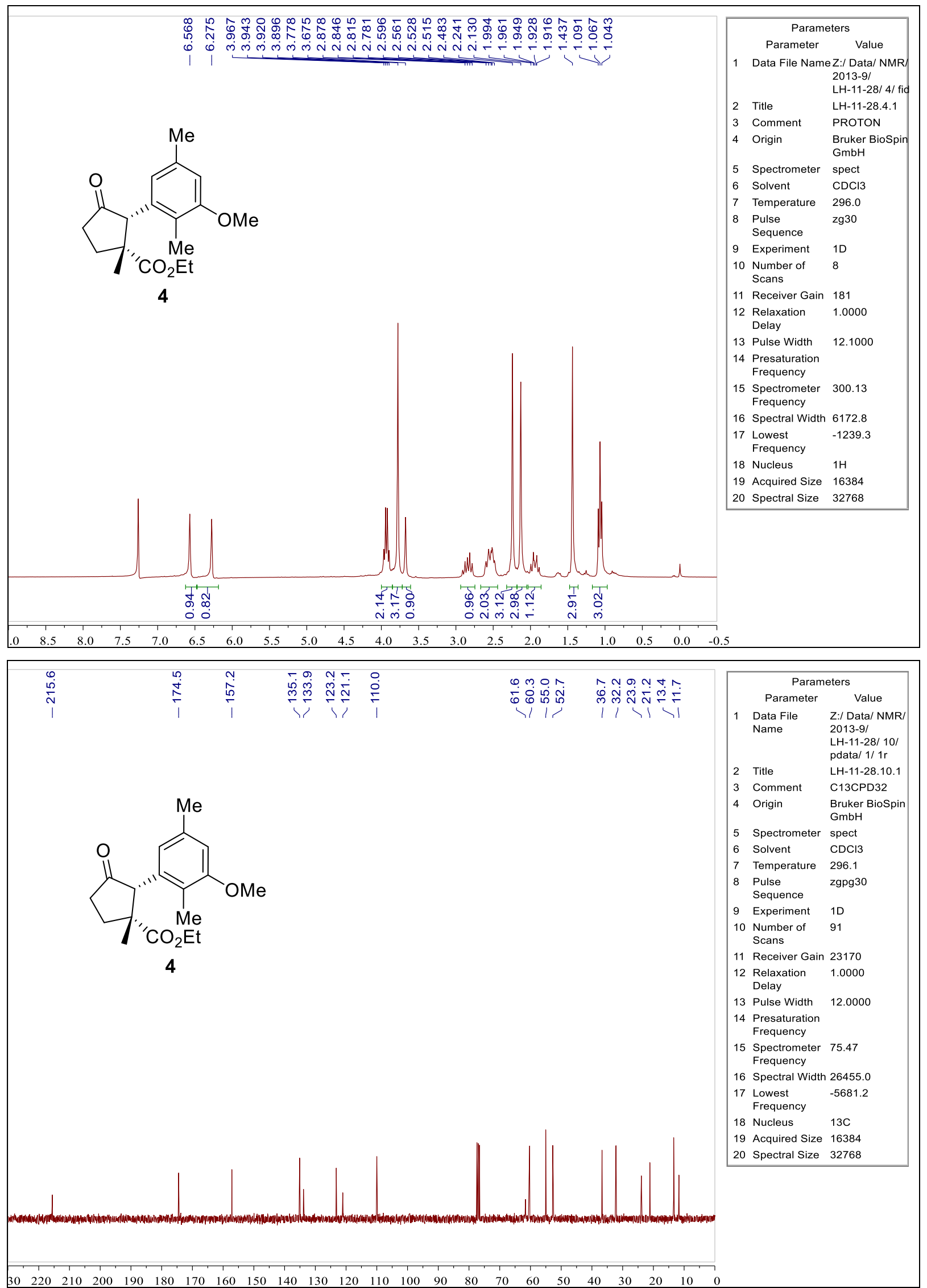
Compound 16.
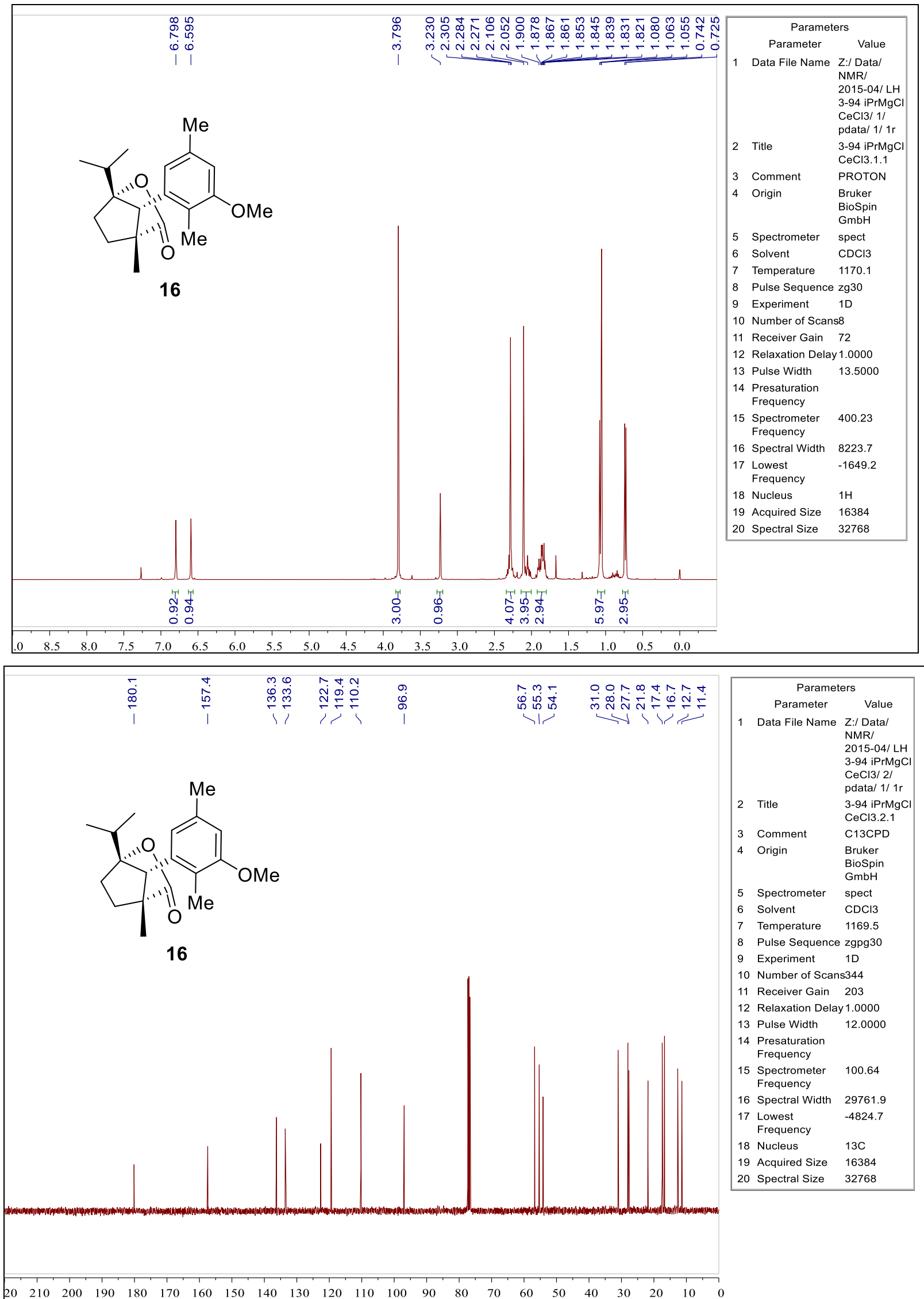
Compound 19.

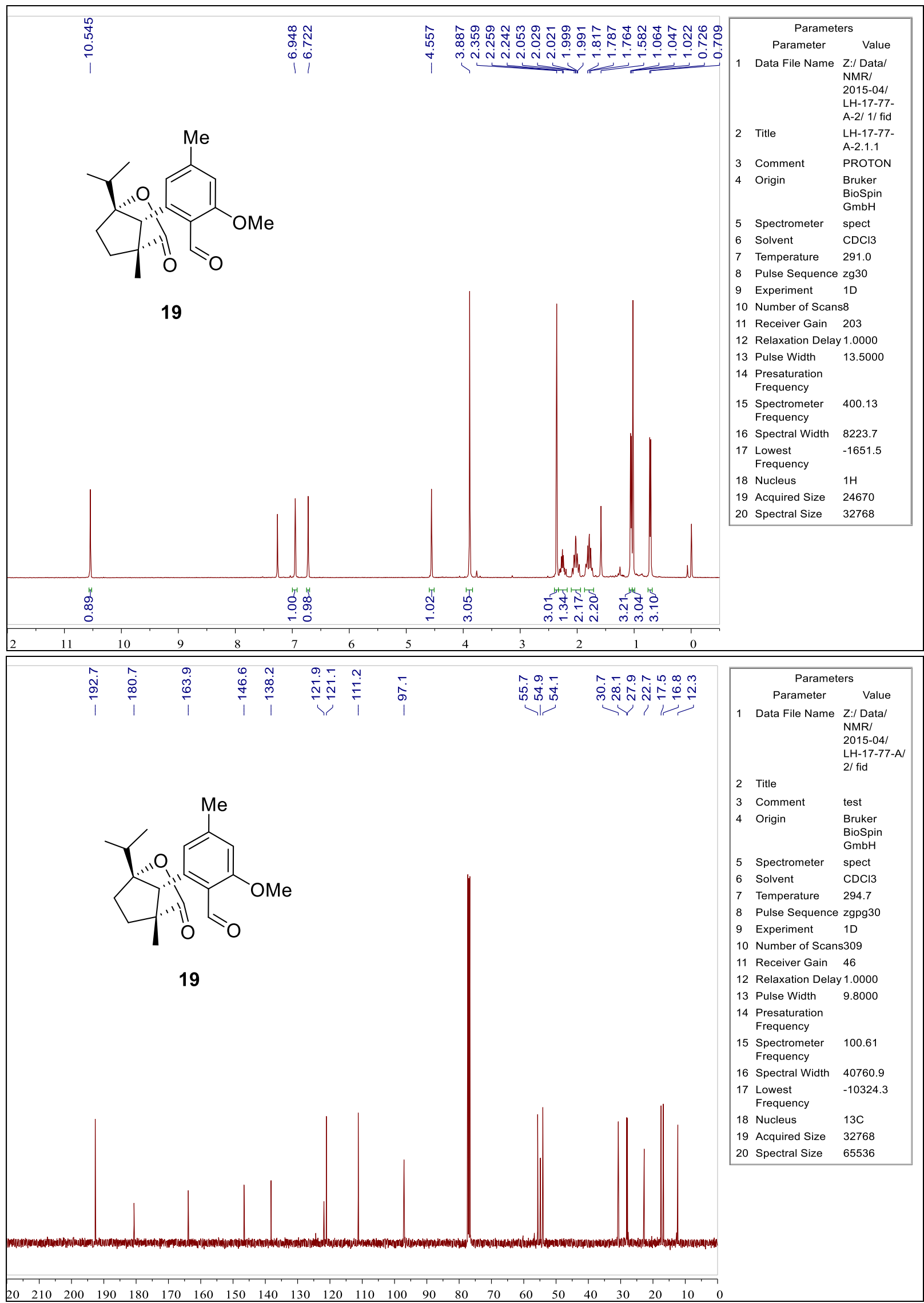


Compound 20.

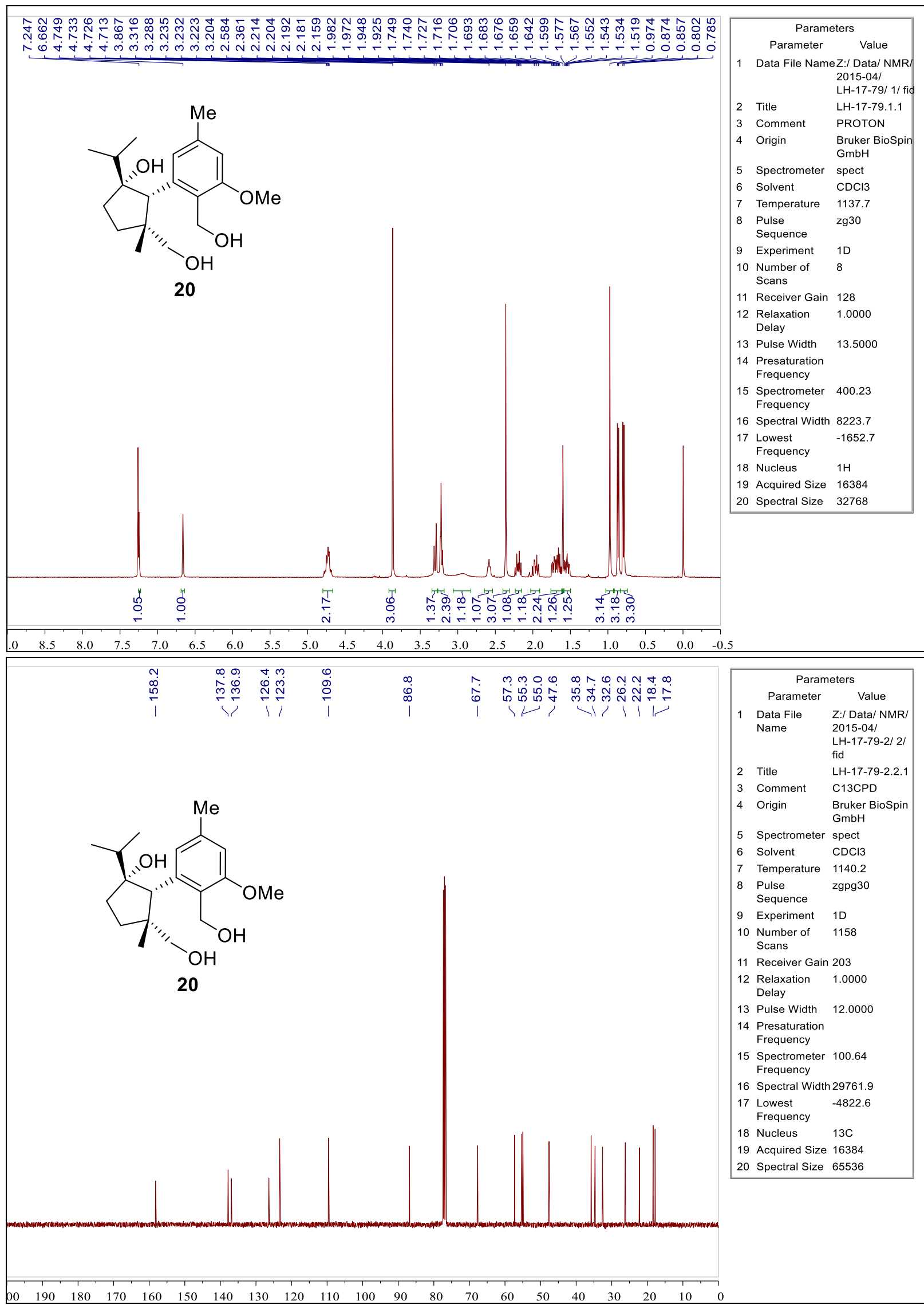




\section{Compound 21.}

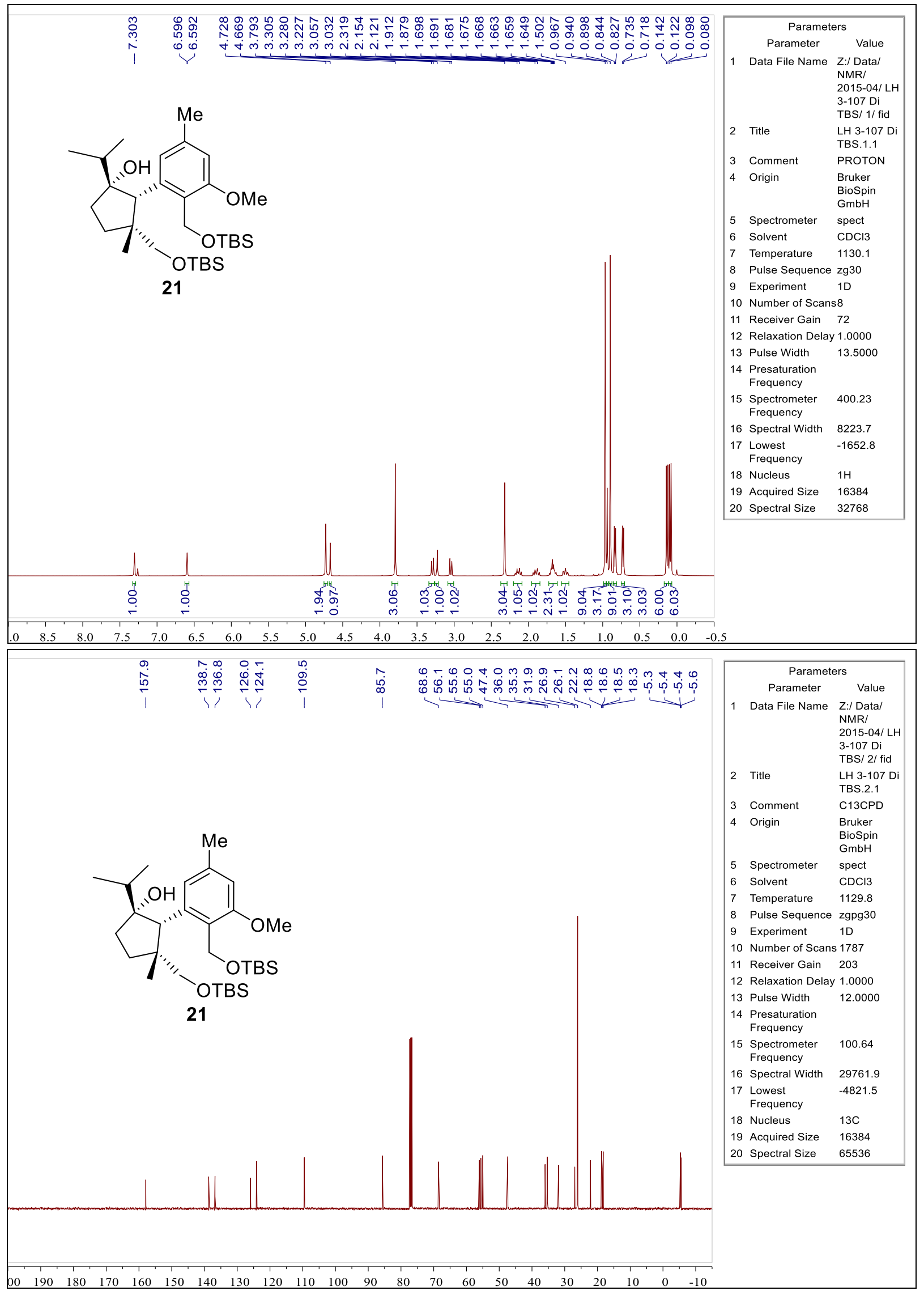




\section{Compound 22a.}

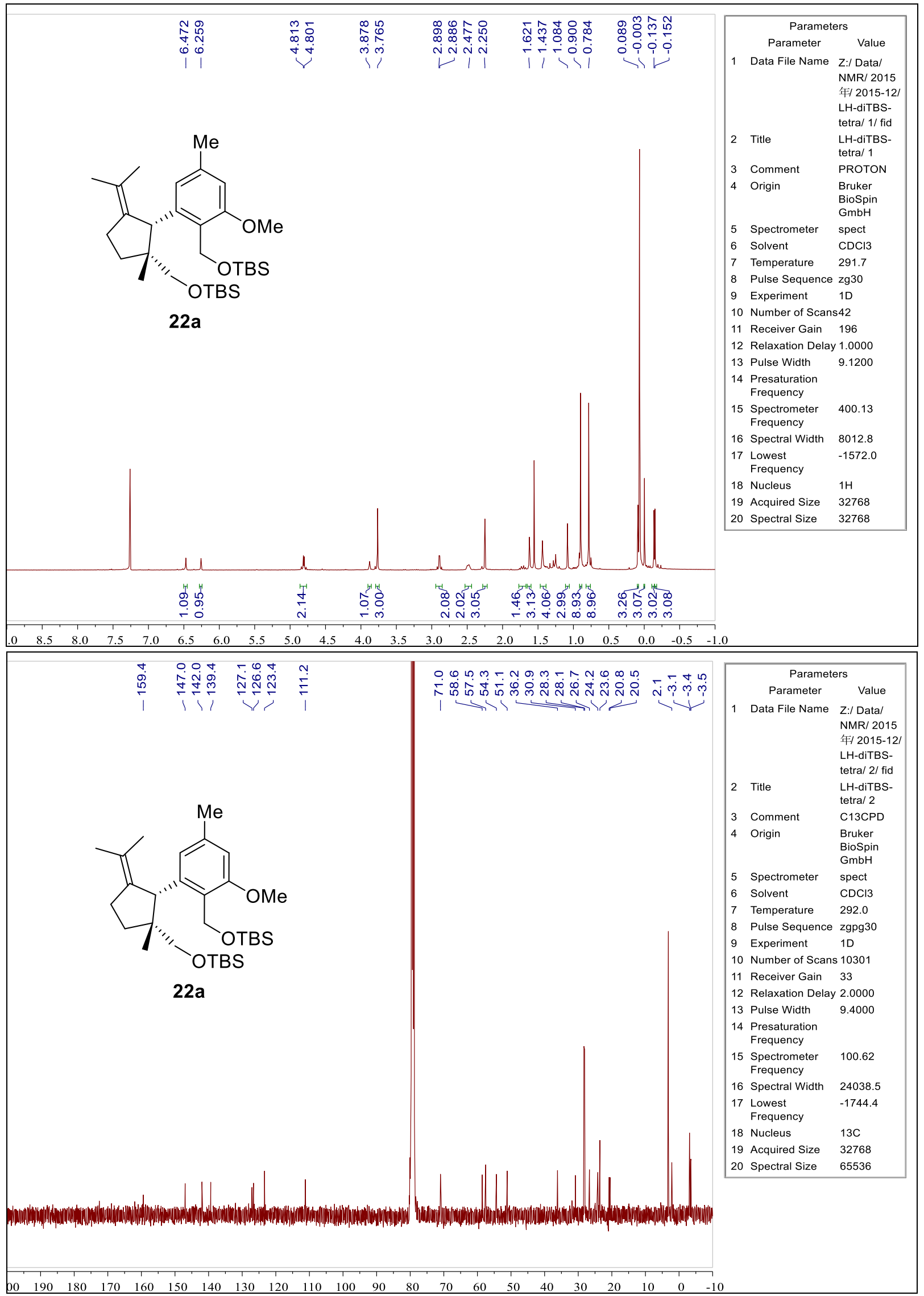




\section{Compound 22b.}
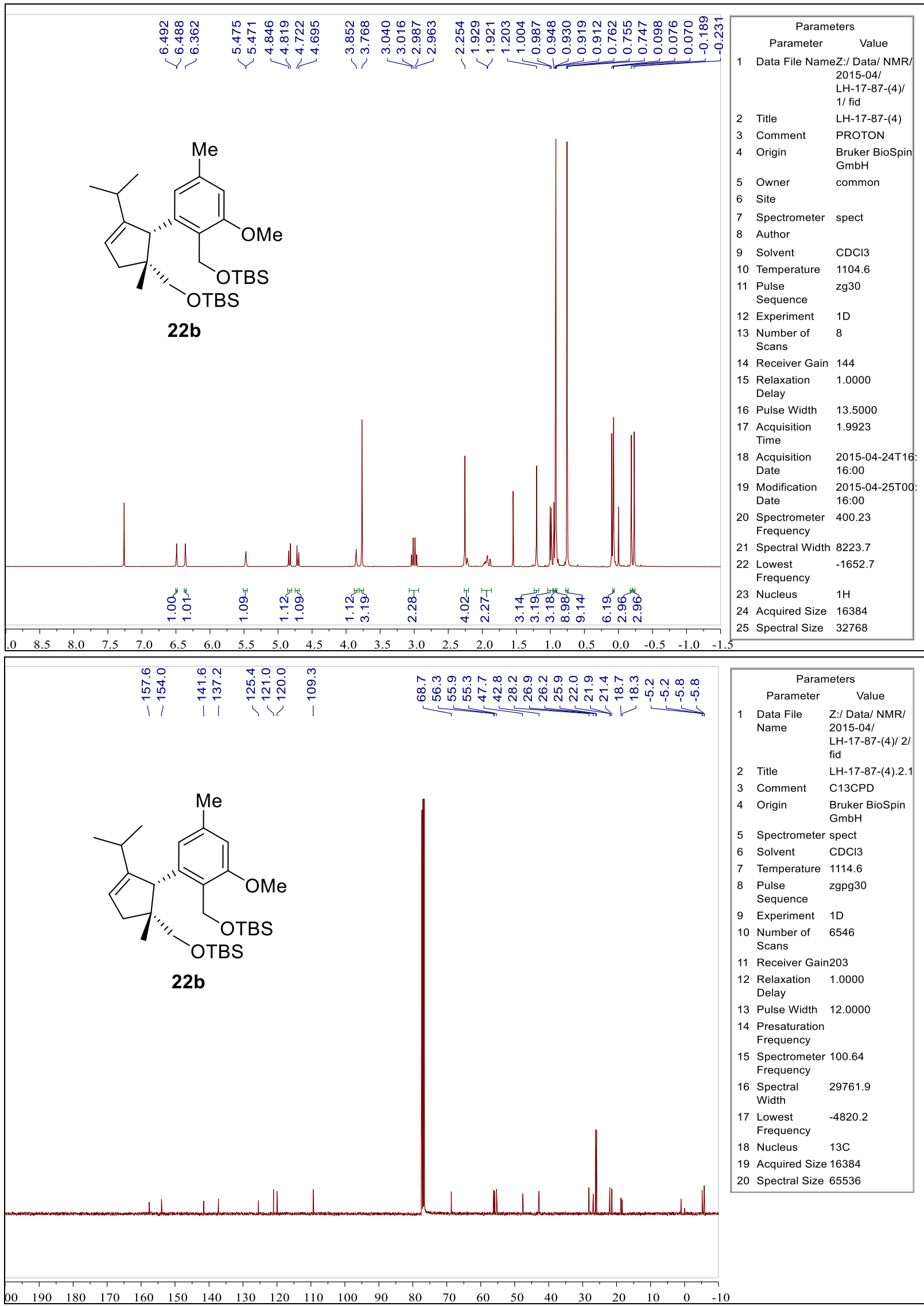


\section{Compound 23.}

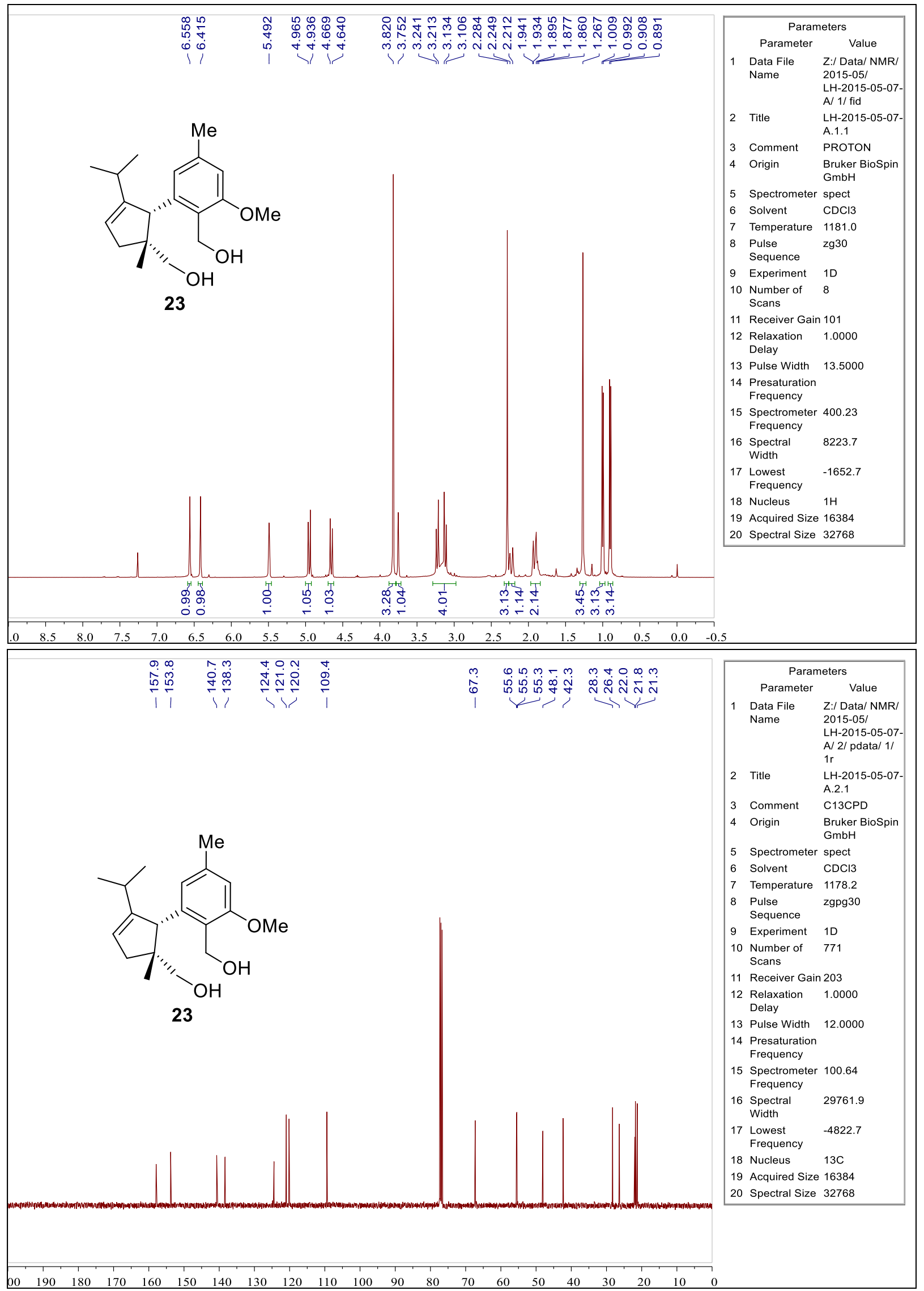


Compound 5.

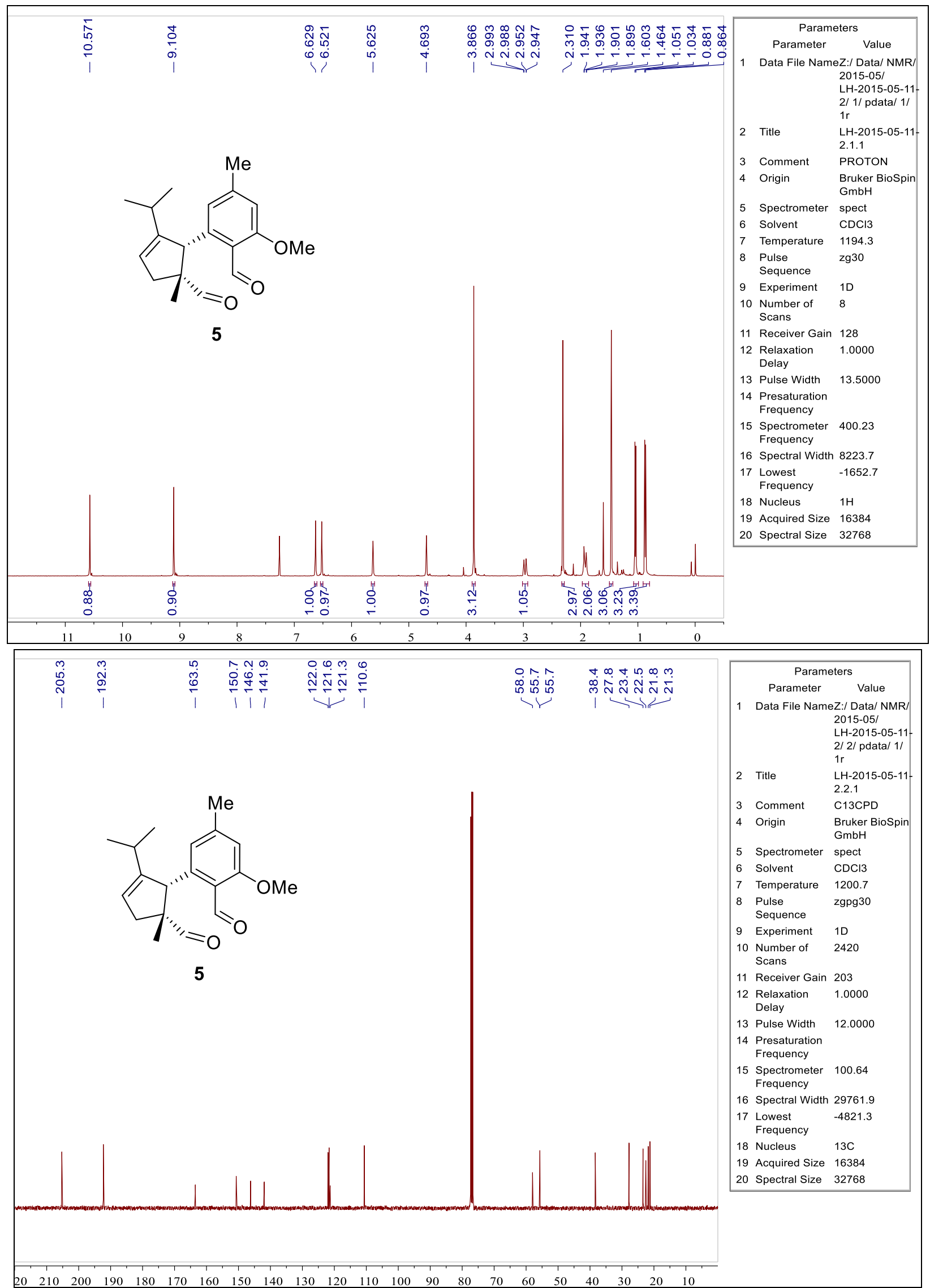




\section{Compound 6.}

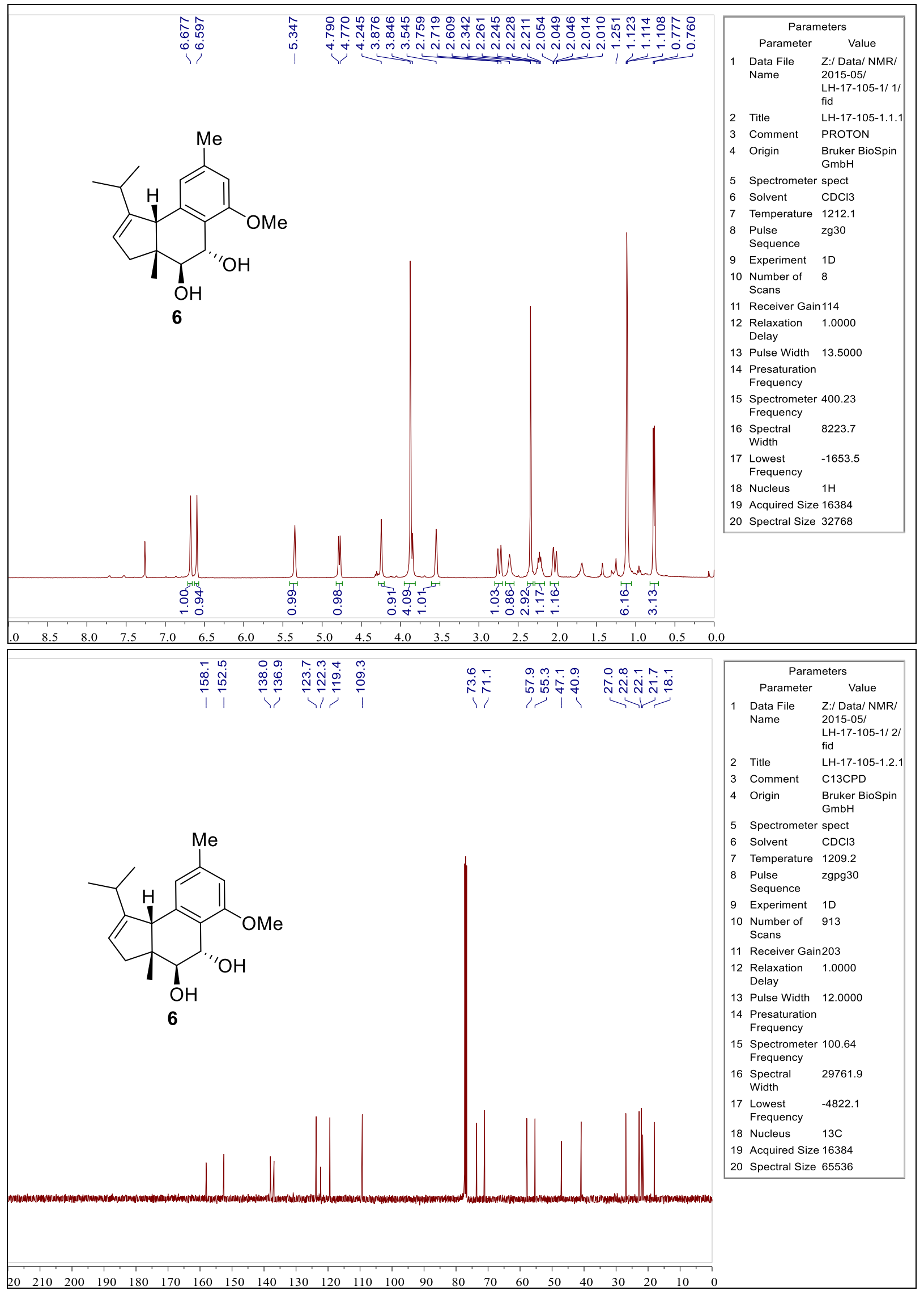




\section{Compound 25.}

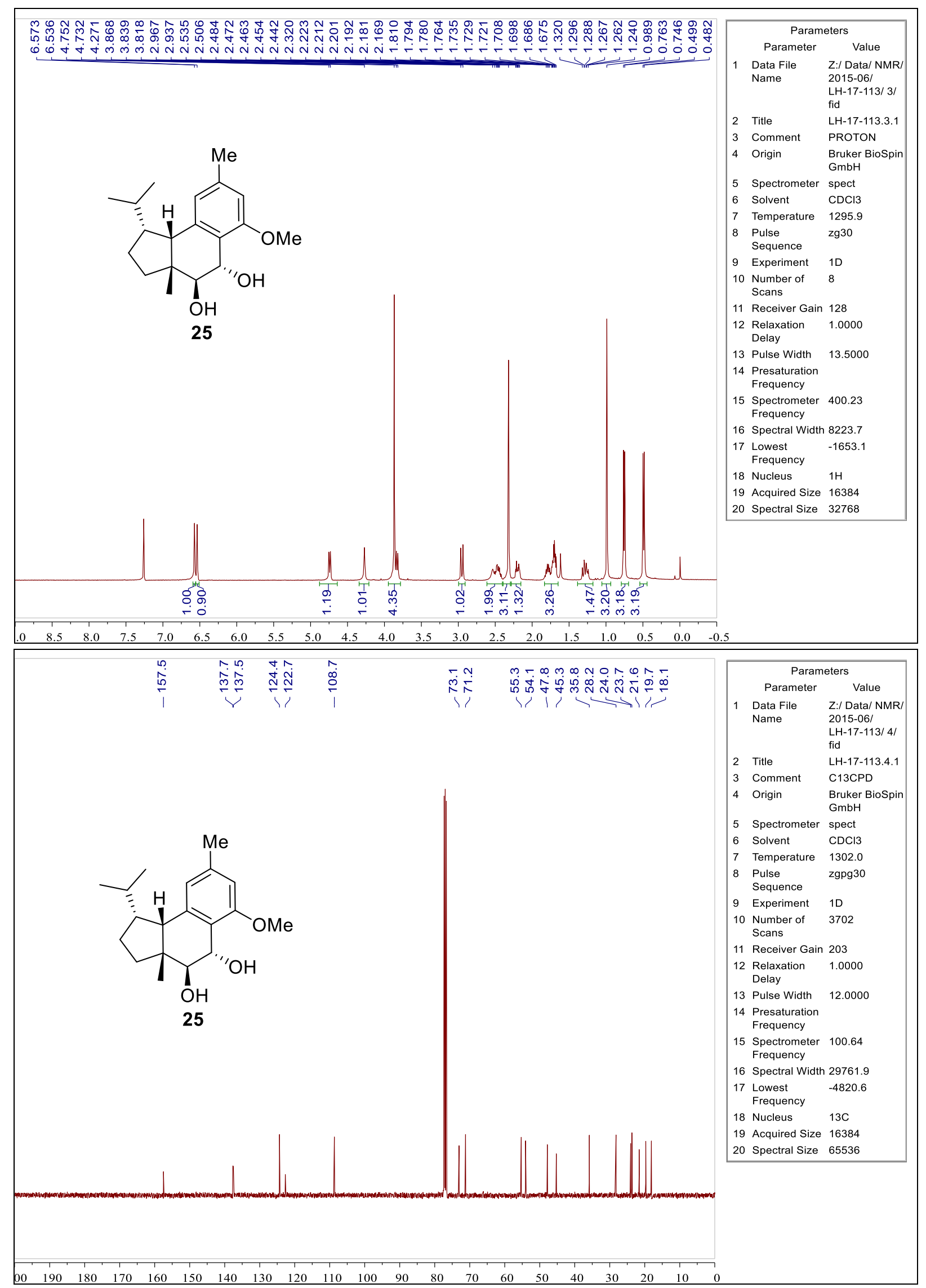




\section{Compound 26.}

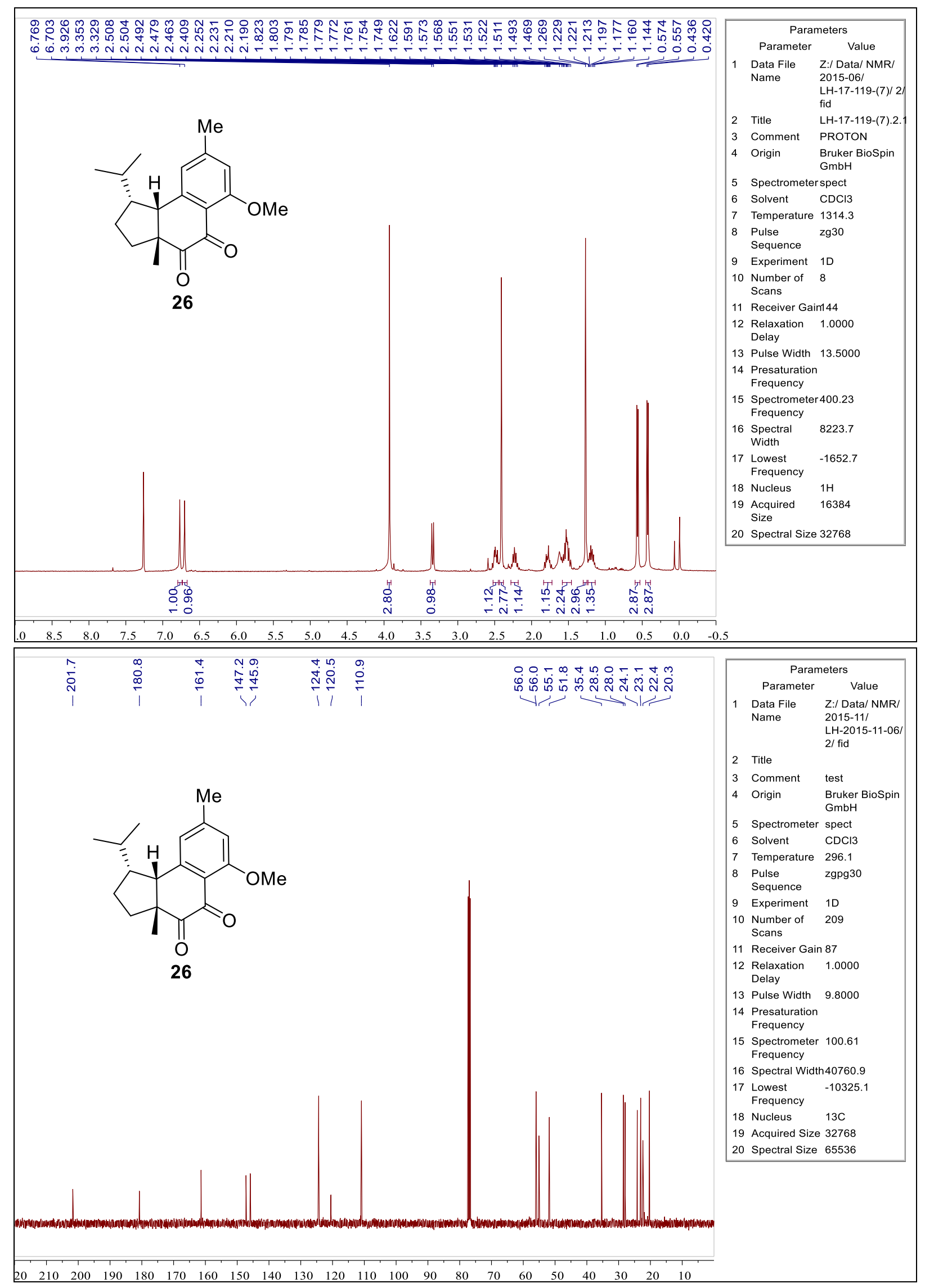


Compound 27.
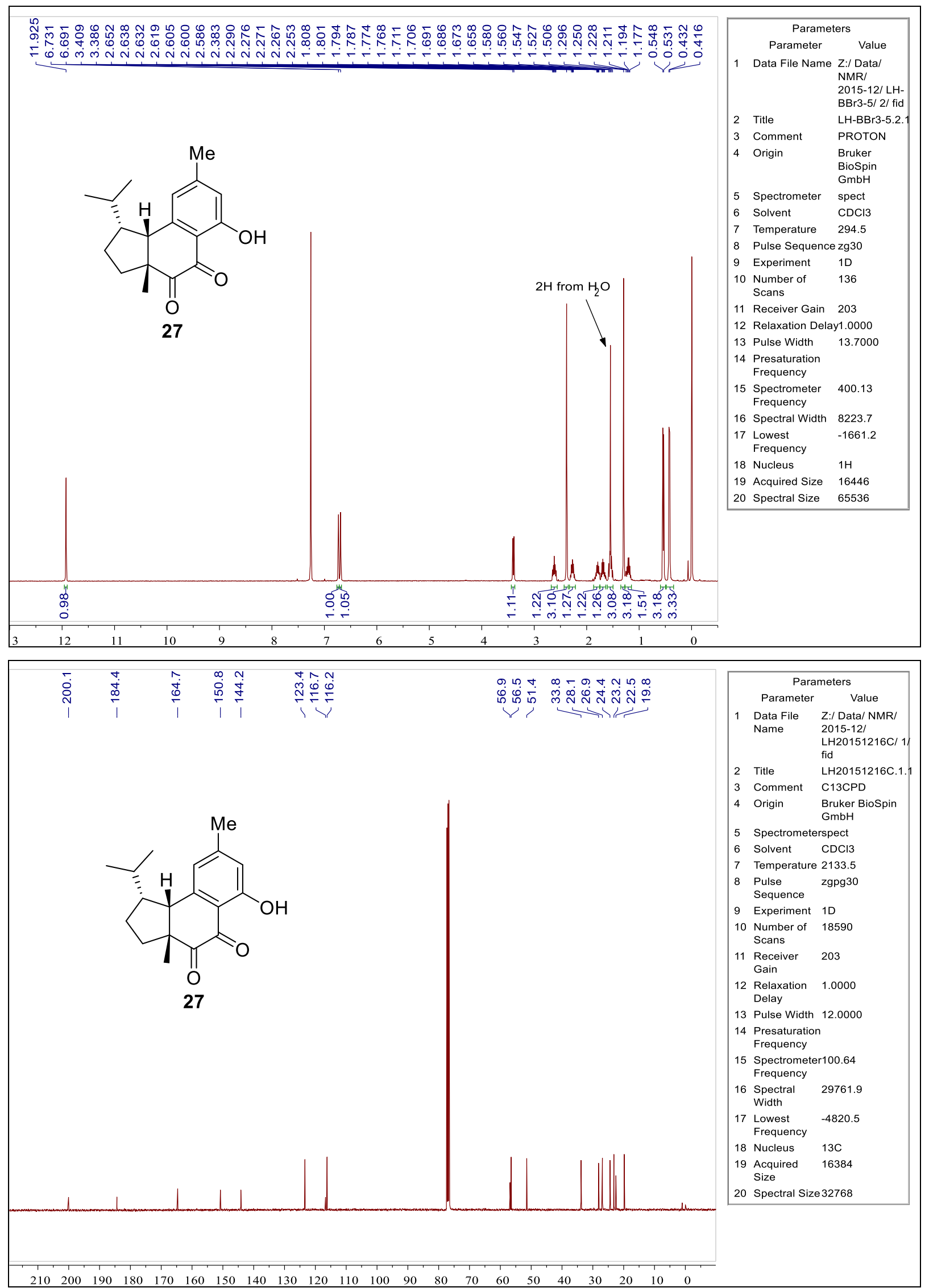


\section{(-)-Hamigeran B}

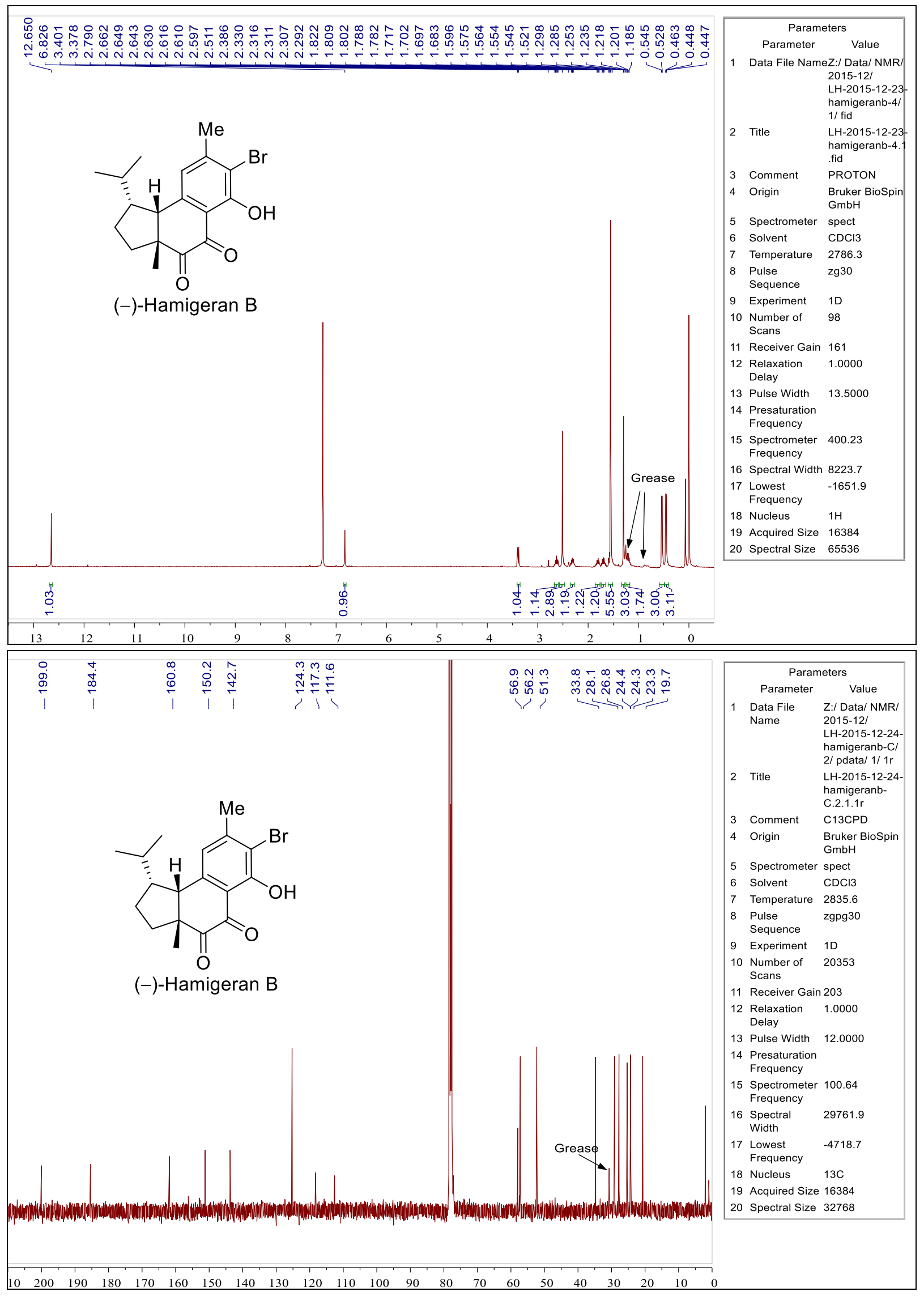




\section{(-)-4-Bromhamigeran B}

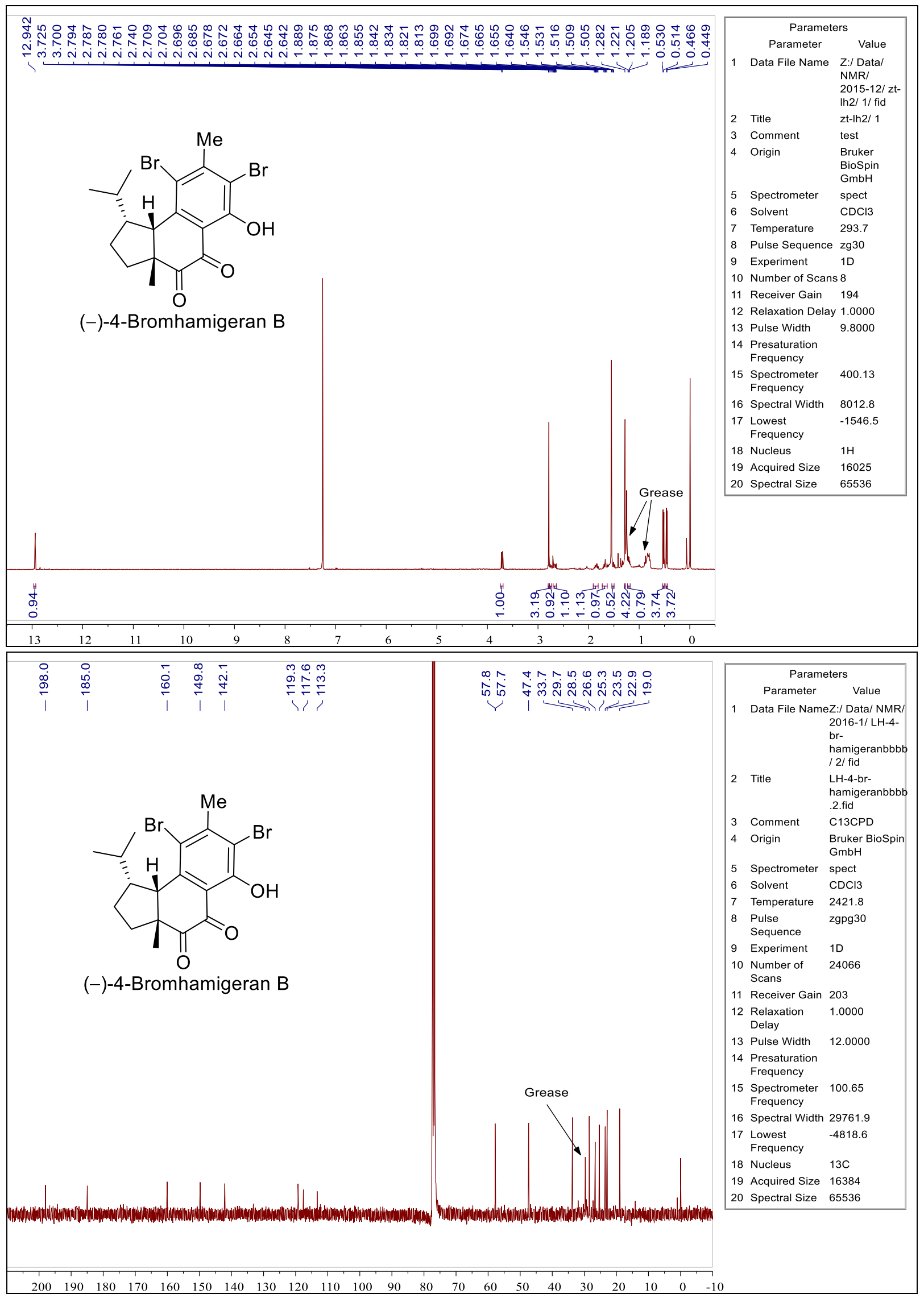


Compound 14.

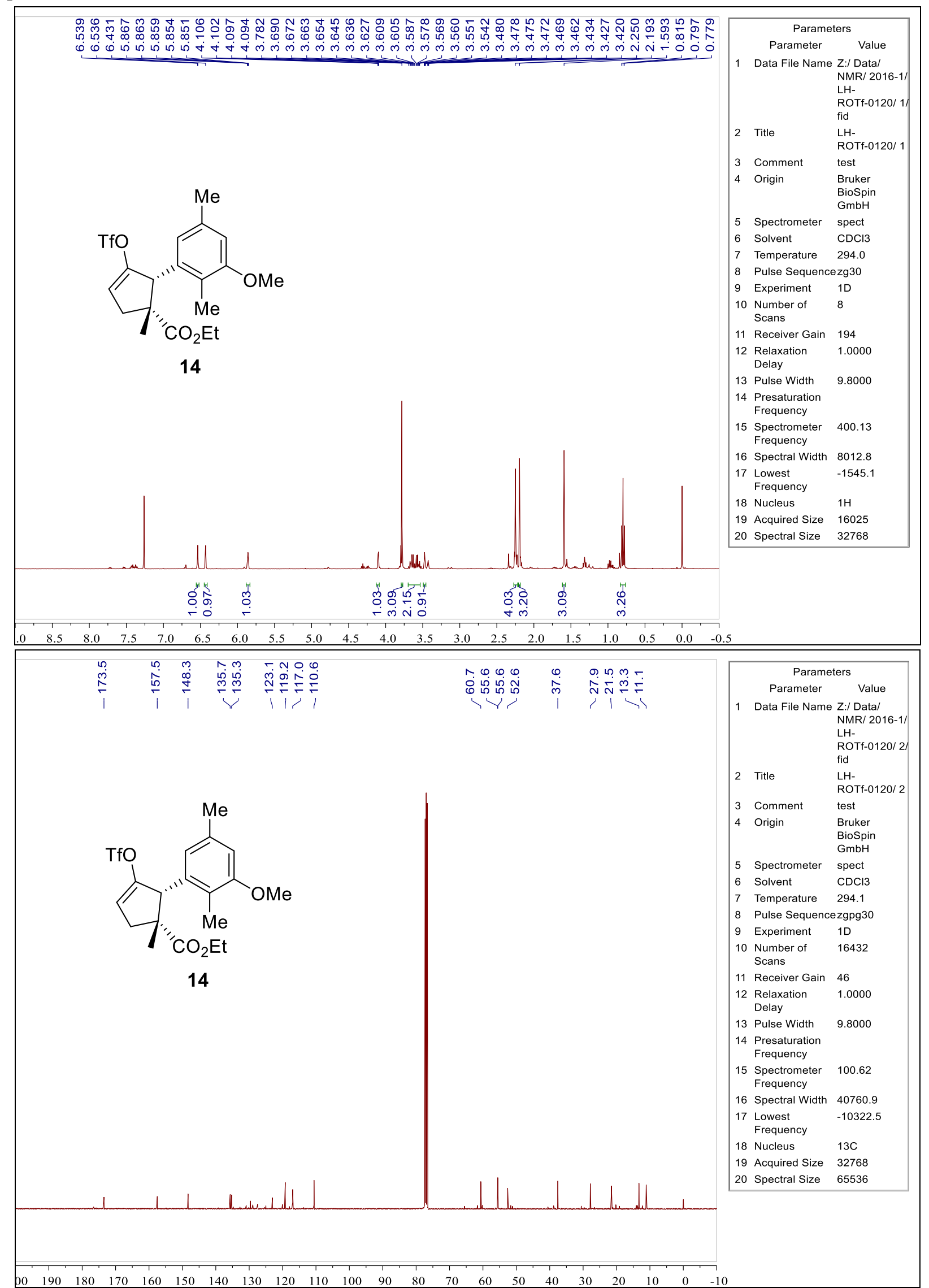




\section{Compound 17.}
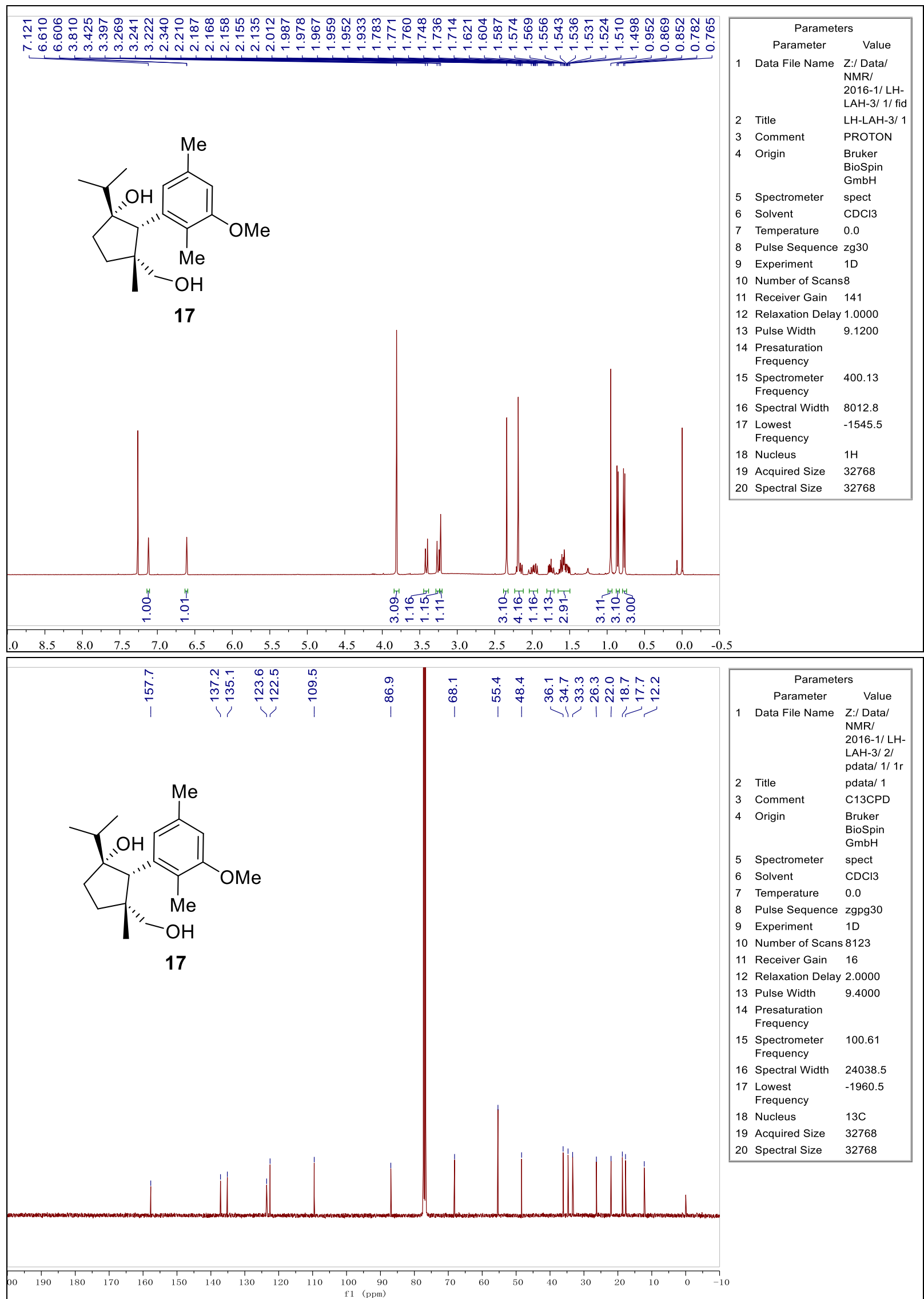


\section{(F) HPLC Charts}

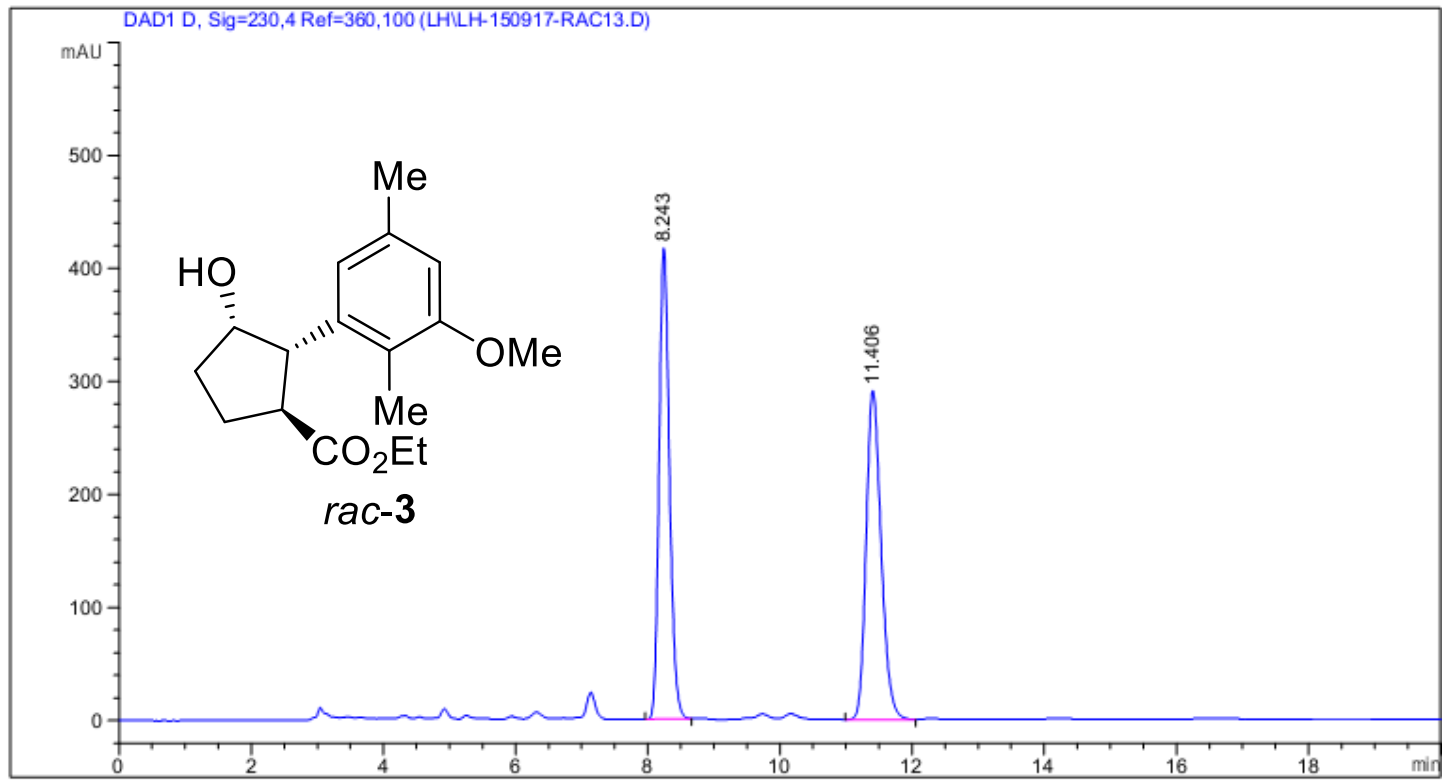

\begin{tabular}{|c|c|c|c|c|c|c|}
\hline $\begin{array}{c}\text { eak } \\
\#\end{array}$ & $\begin{array}{c}\text { RetTime } \\
\text { [min] }\end{array}$ & Type & $\begin{array}{l}\text { Width } \\
\text { [min] }\end{array}$ & $\begin{array}{c}\text { Area } \\
{\left[\mathrm{mAU}{ }^{\star} \mathrm{s}\right]}\end{array}$ & $\begin{array}{l}\text { Height } \\
\text { [mAU] }\end{array}$ & $\begin{array}{c}\text { Area } \\
\frac{8}{b}\end{array}$ \\
\hline & & & & --- & -- & . \\
\hline 1 & & $B$ & 0.1673 & 4552.67676 & 417.17252 & 602 \\
\hline 2 & 11.406 & RR & .2424 & 4596.54736 & 290.85916 & 398 \\
\hline
\end{tabular}

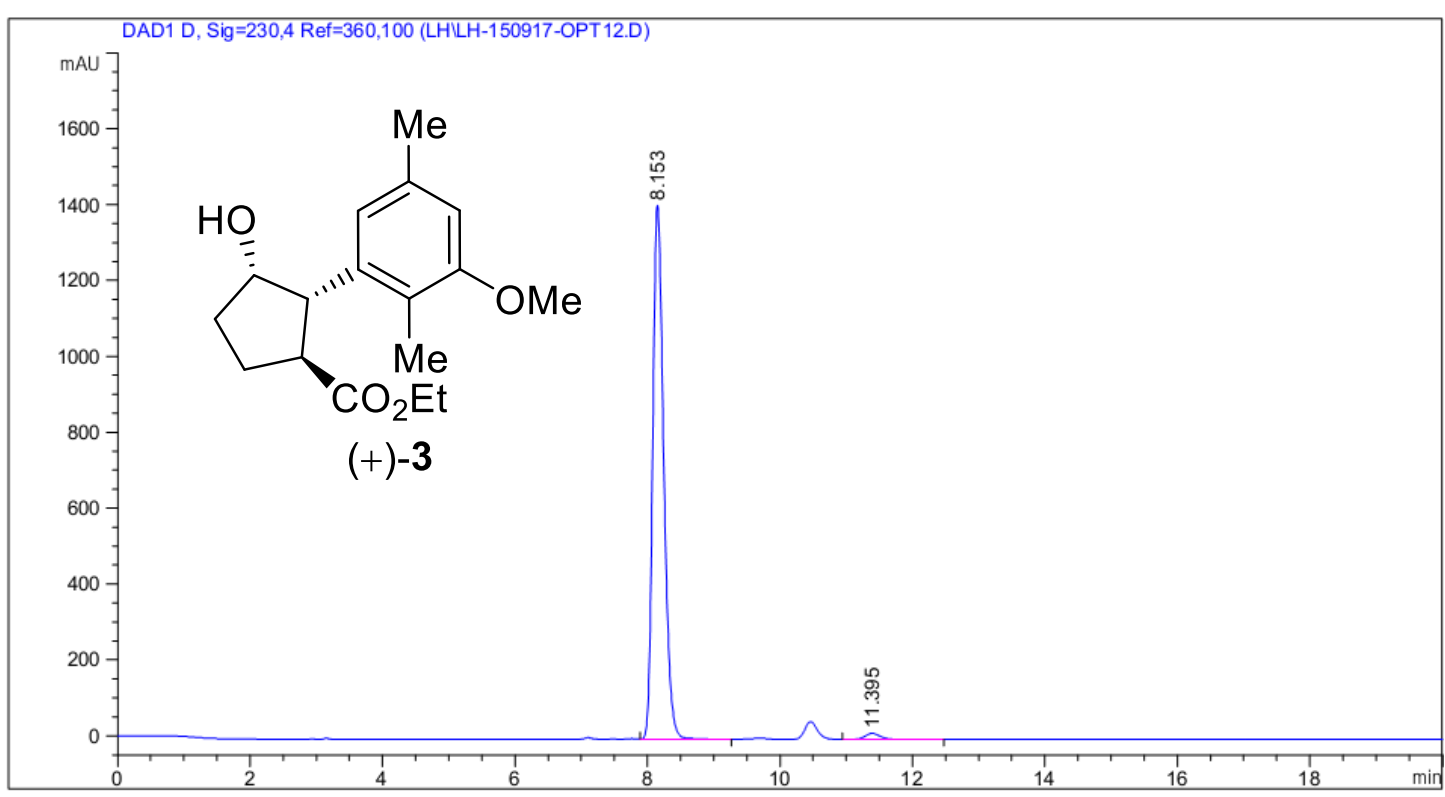

\begin{tabular}{|c|c|c|c|c|c|c|}
\hline $\begin{array}{c}\text { Peak } \\
\#\end{array}$ & $\begin{array}{c}\text { RetTime } \\
\text { [min] }\end{array}$ & Type & $\begin{array}{l}\text { Width } \\
\text { [min] }\end{array}$ & $\begin{array}{c}\text { Area } \\
{\left[\mathrm{mAU}{ }^{*} \mathrm{~s}\right]}\end{array}$ & $\begin{array}{l}\text { Height } \\
\text { [mAU] }\end{array}$ & $\begin{array}{c}\text { Area } \\
\quad \frac{\circ}{0}\end{array}$ \\
\hline & & & & & |---- & \\
\hline 1 & & 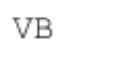 & & $1.61398 \mathrm{e} 4$ & 1406.6 & \\
\hline 2 & 1.395 & & 51 & 250.36818 & 112 & 275 \\
\hline
\end{tabular}

